\title{
ELLIPTIC MINUSCULE PAIRS AND SPLITTING ABELIAN VARIETIES
}

\author{
V. KUMAR MURTY AND YING ZONG
}

\section{INTRODUCTION : WHY ELLIPTIC MINUSCULE PAIRS}

The motivation of this article was to study the following question (cf. [8], 1.1) through a monodromy approach.

Question 1.1. Let $A_{K}$ be an absolutely simple abelian variety over a number field $K$. Does there exist a finite extension $L$ of $K$ such that the base change of $A_{K}$ to each finite extension of $L$ has simple specializations at a set of places of positive density?

Let us recall some notions before we formulate this question in more precise terms and impose a natural hypothesis on $A_{K}$.

Let $t=\operatorname{Spec}(K), \bar{t}$ a geometric point of $t$ and $S$ a dense open subscheme of the normalization of $\operatorname{Spec}(\mathbf{Z})$ in $t$ such that $A_{t}=A_{K}$ extends to an abelian scheme $A$ over $S$.

We call an arbitrary $S$-fiber of $A$ a specialization of $A_{t}$. A specialization $A_{s}=A \times_{S} s$ at a point $s$ of $S$ is said to be simple if it is a simple object in the category of $s$-abelian varieties up to isogenies, that is, if $\operatorname{End}_{s}\left(A_{s}\right) \otimes_{\mathbf{z}} \mathbf{Q}$ is a $\mathbf{Q}$-division algebra. And, a specialization $A_{s}$ is absolutely simple if $A_{s} \times_{s} \bar{s}$ is simple for some geometric point $\bar{s}$ of $s$.

Recall that a subset $\Xi$ of $S \backslash\{t\}$ has (natural) density $d$ ([10], I-7), $0 \leq d \leq 1$, if asymptotically in $N \in \mathbf{R}$,

$$
\operatorname{Card}(\{s \in \Xi, \operatorname{Card}(k(s)) \leq N\})=d \frac{N}{\log N}+o\left(\frac{N}{\log N}\right) .
$$

As a fundamental example, the set

$$
\{s \in S \backslash\{t\}, k(s) \text { is a prime field }\}
$$

has density 1 .

In general, the density of $\Xi$ is taken here to be the supremum of the densities of its "measurable" subsets.

What we asked above is whether there exists some finite extension $L$ of $K$ such that for each finite extension $K^{\prime}$ of $L$, if $S^{\prime}$ denotes the 
normalization of $S$ in $t^{\prime}=\operatorname{Spec}\left(K^{\prime}\right)$, the set

$$
\left\{s^{\prime} \in S^{\prime} \backslash\left\{t^{\prime}\right\}, A \times_{S} s^{\prime} \text { is simple }\right\},
$$

or what amounts to the same, the subset

$$
\left\{s^{\prime} \in S^{\prime} \backslash\left\{t^{\prime}\right\}, k\left(s^{\prime}\right) \text { is a prime field, } A \times_{S} s^{\prime} \text { is simple }\right\}
$$

has positive density.

Note that

Lemma 1.2. The algebra $\operatorname{End}_{t^{\prime}}\left(A_{t^{\prime}}\right) \otimes_{\mathbf{Z}} \mathbf{Q}$ must be a field if $A_{t^{\prime}}$ has at least one simple specialization $A_{s^{\prime}}$ at a point $s^{\prime}$ with finite prime residue field.

Proof. For, $\operatorname{End}_{s^{\prime}}\left(A_{s^{\prime}}\right) \otimes_{\mathbf{Z}} \mathbf{Q}$ is a field at such a point $s^{\prime}([13]$, p. 98, line 1) and the specialization homomorphism

$$
s p: \operatorname{End}_{t^{\prime}}\left(A_{t^{\prime}}\right)=\operatorname{End}_{S^{\prime}}\left(A_{S^{\prime}}\right) \hookrightarrow \operatorname{End}_{s^{\prime}}\left(A_{s^{\prime}}\right)
$$

is injective.

In particular, our question has a negative answer unless

$$
E:=\operatorname{End}_{\bar{t}}\left(A_{\bar{t}}\right) \otimes_{\mathbf{z}} \mathbf{Q}
$$

is a field, as was predicted in [8] and known to J. Achter in a less precise way ([1], Theorem B).

One can ask if the hypothesis that $E=\operatorname{End}_{\bar{t}}\left(A_{\bar{t}}\right) \otimes_{\mathbf{z}} \mathbf{Q}$ be a field is sufficient for the question to have a positive answer (cf. 1.5, 1.7).

For this, enlarge if necessary $K$ to a finite extension so that

$$
\operatorname{End}_{t}\left(A_{t}\right)=\operatorname{End}_{\bar{t}}\left(A_{\bar{t}}\right) \text {. }
$$

Let $\ell$ be a prime number and let $\mathfrak{l}$ be a place of $E$ above $\ell$. Replacing $S$ by its open sub-scheme $S[1 / \ell]$ if necessary, we assume that $\ell$ is prime to the residue characteristics of $S$. Choose for each closed point $s \in S$ a geometric point $\bar{s}$ located at $s$ and a "chemin" $c h_{s}$ connecting $\bar{s}$ to $\bar{t}$ (SGA 1, Éxposé V, 7). Let $F_{s} \in \pi_{1}(s, \bar{s})$ be the geometric Frobenius and $F_{s}^{*}$ the image of $F_{s}$ under the composition

$$
\pi_{1}(s, \bar{s}) \rightarrow \pi_{1}(S, \bar{s}) \stackrel{c h_{s}}{\longrightarrow} \pi_{1}(S, \bar{t}) \stackrel{\rho_{\ell, \bar{t}}}{\longrightarrow} \mathrm{GL}_{E}\left(H^{1}\left(A_{\bar{t}}, \mathbf{Q}_{\ell}\right)\right),
$$

where $\rho_{\ell, \bar{t}}$ is the $\ell$-adic monodromy representation associated with the abelian scheme $A$. Let $M_{\ell}=\operatorname{Im}\left(\rho_{\ell, \bar{t}}\right)$ be the monodromy and $M_{\ell}^{\text {Zar }}$ its Zariski closure in $\mathrm{GL}_{E}\left(H^{1}\left(A_{\bar{t}}, \mathbf{Q}_{\ell}\right)\right)$. Further enlarging $K$ to a finite extension if necessary, one may assume that $M_{\ell}^{\text {Zar }}$ is connected.

The group $M_{\ell}^{\mathrm{Zar}}$ is then by Faltings ([5], satz 3 ) reductive and (loc.cit., satz 4)

$$
\operatorname{End}_{t}\left(A_{t}\right) \otimes_{\mathbf{z}} \mathbf{Q}_{\ell} \widetilde{\rightarrow} \operatorname{End}_{M_{\ell}^{\mathrm{Zar}}}\left(H^{1}\left(A_{\bar{t}}, \mathbf{Q}_{\ell}\right)\right)^{\text {opposite }}
$$




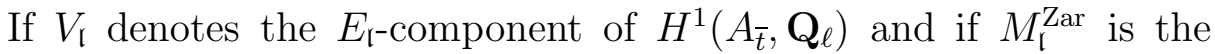
image of $M_{\ell}^{\text {Zar }}$ in $\mathrm{GL}_{E_{\mathrm{l}}}\left(V_{\mathrm{l}}\right)$, one has

$$
E_{\mathfrak{l}}=\operatorname{End}_{M_{\mathfrak{l}}^{\text {Zar }}}\left(V_{\mathfrak{l}}\right),
$$

which amounts to the absolute irreducibility of $V_{\mathfrak{l}}$ as a $E_{\mathfrak{l}}$-linear representation of $M_{\mathfrak{l}}^{\text {Zar }}$. The image $M_{\mathfrak{l}}$ of $M_{\ell}$ in $M_{\mathfrak{l}}^{\text {Zar }}\left(E_{\mathfrak{l}}\right)$ is an open analytic subgroup by Bogomolov [2].

At each closed point $s$ of $S$, the commutant of $F_{s}^{*}$ on $V_{\mathfrak{l}}$ is

$$
\left(\operatorname{End}_{s}\left(A_{s}\right) \otimes_{\mathbf{Z}} \mathbf{Q}\right)^{\text {opposite }} \otimes_{E} E_{\mathfrak{l}}
$$

as by Tate 12

$$
\operatorname{End}_{s}\left(A_{s}\right) \otimes_{\mathbf{z}} \mathbf{Q}_{\ell} \stackrel{\rightarrow}{\rightarrow} \operatorname{End}_{F_{s}^{*}}\left(H^{1}\left(A_{\bar{t}}, \mathbf{Q}_{\ell}\right)\right)^{\text {opposite }}
$$

Recall that if at one point $s \in S \backslash\{t\}$ with prime residue field $A_{s}$ is simple, then

$$
\operatorname{End}_{s}\left(A_{s}\right) \otimes_{\mathbf{z}} \mathbf{Q}=\mathbf{Q}\left(F_{s}^{*}\right)
$$

is a field of degree $2 g$ over $\mathbf{Q}$, where $g=\operatorname{dim}\left(A_{t}\right)$. This Frobenius $F_{s}^{*}$ has all distinct eigenvalues on $H^{1}\left(A_{\bar{t}}, \mathbf{Q}_{\ell}\right)$ and $F_{s}^{*}$ lies in a unique maximal torus of $M_{\ell}^{\text {Zar }}$. So

Lemma 1.3. If $A_{t}$ has at least one simple specialization at a point $s$ with finite prime residue field, then some, hence every, maximal torus of $M_{\ell}^{\text {Zar }}$ acts on $H^{1}\left(A_{\bar{t}}, \mathbf{Q}_{\ell}\right)$ without multiple weights.

This weight multiplicity free condition has the following immediate implication :

Proposition 1.4. Suppose that $M_{\ell}^{\mathrm{Zar}}$ is connected and that the monodromy representation $H^{1}\left(A_{\bar{t}}, \mathbf{Q}_{\ell}\right)$ has no multiple weights. Then in a density 1 set $\Sigma$ of points $s \in S \backslash\{t\}$ every positive power of $F_{s}^{*}$ has all distinct eigenvalues on $H^{1}\left(A_{\bar{t}}, \mathbf{Q}_{\ell}\right)$. In particular, the simple factors of each $A_{s}, s \in \Sigma$, are absolutely simple mutually non-isogenous over $\bar{s}$ and $\operatorname{End}_{\bar{s}}\left(A_{\bar{s}}\right)$ is commutative. A specialization at a point $s \in \Sigma$ is thus absolutely simple if it is simple.

Proof. Let $s$ be a point of $S \backslash\{t\}$. The Frobenius $F_{s}^{*}$ being semi-simple on $H^{1}\left(A_{\bar{t}}, \mathbf{Q}_{\ell}\right)$ lies in a maximal torus $\mathfrak{T}(s)$ of $M_{\ell}^{\text {Zar }}$, as $M_{\ell}^{\text {Zar }}$ is connected. And, $F_{s}^{*}$ has eigenvalues $\chi_{i}\left(F_{s}^{*}\right)$, where $\chi_{i}$ are the weights of $H^{1}\left(A_{\bar{t}}, \mathbf{Q}_{\ell}\right)$ relative to $\mathfrak{T}(s)$.

These eigenvalues generate over $\mathbf{Q}$ an extension of degree bounded by a constant, as the characteristic polynomial of $F_{s}^{*}$ has coefficients in $\mathbf{Z}$ (Weil). Thus, if some ratio $\chi_{i}\left(F_{s}^{*}\right) / \chi_{j}\left(F_{s}^{*}\right)$ is a root of unity, its order divides an integer $N(g)>1$ depending only on $g=\operatorname{dim}\left(A_{t}\right)$. 
The following subset of $M_{\ell}$

$$
\left\{u \in M_{\ell}, u^{N(g)} \text { has all distinct eigenvalues on } H^{1}\left(A_{\bar{t}}, \mathbf{Q}_{\ell}\right)\right\}
$$

is Zariski open and stable under conjugation. Its volume in the normalized Haar measure of $M_{\ell}$ is by Cebotarev's density theorem ([10], I-8, Corollary 2) the density of the set

$$
\left\{s \in S \backslash\{t\},\left(F_{s}^{*}\right)^{N(g)} \text { has all distinct eigenvalues }\right\}
$$

or the density of the set

$$
\Sigma=\left\{s \in S \backslash\{t\},\left(F_{s}^{*}\right)^{N} \text { has all distinct eigenvalues, } \forall N \geq 1\right\} .
$$

This volume and this density are 1 because the characters $\chi_{i}$ are all distinct by assumption.

Consider an integer $N \geq 1$ and a finite extension $k^{\prime}$ of $k(s)$ of degree $N$, where $s \in \Sigma$. Put $s^{\prime}=\operatorname{Spec}\left(k^{\prime}\right)$ and $A_{s^{\prime}}=A_{s} \times{ }_{s} s^{\prime}$. As $\left(F_{s}^{*}\right)^{N}$ has all distinct eigenvalues on $H^{1}\left(A_{\bar{t}}, \mathbf{Q}_{\ell}\right)$, the ring $\operatorname{End}_{s^{\prime}}\left(A_{s^{\prime}}\right)$ is commutative, for by Tate

$$
\operatorname{End}_{s^{\prime}}\left(A_{s^{\prime}}\right) \otimes_{\mathbf{Z}} \mathbf{Q}_{\ell} \widetilde{\rightarrow} \operatorname{End}_{\left(F_{s}^{*}\right)^{N}}\left(H^{1}\left(A_{\bar{t}}, \mathbf{Q}_{\ell}\right)\right)^{\text {opposite }}
$$

Now $A_{s}$ is isogenous to a product of simple abelian varieties $A_{i}, i \in I$. If one factor appears with multiplicity $>1$, or if $A_{i} \times_{s} s^{\prime}$ is not simple, or if $A_{i} \times_{s} s^{\prime}$ and $A_{j} \times{ }_{s} s^{\prime}$ are isogenous for $i \neq j$, then $\operatorname{End}_{s^{\prime}}\left(A_{s^{\prime}}\right)$ is not commutative. So these factors $A_{i}$ of $A_{s}$ are absolutely simple mutually non-isogenous over $\bar{s}, \forall s \in \Sigma$.

And, this weight multiplicity free condition means $([6], 4.6 .3)$ that the tensor components of each $V_{\mathfrak{l}}$, as a $E_{\mathfrak{l}}$-linear representation of the derived group of $M_{\mathfrak{l}}^{\text {Zar }}$, are

- either minuscule

- or of the types $\left(A_{n}, r \omega_{1}\right),\left(A_{n}, r \omega_{n}\right),\left(B_{n}, \omega_{1}\right),\left(C_{3}, \omega_{3}\right),\left(G_{2}, \omega_{1}\right)$ for some integers $n, r>1$.

Recall that a minuscule representation is a highest weight representation all whose weights have the same length.

To seek a positive answer we now assume that some $V_{\mathrm{l}}$ is minuscule. (The types $\left(A_{n}, r \omega_{1}\right),\left(A_{n}, r \omega_{n}\right)$ are not self-dual and thus do not occur in $V_{\mathfrak{l}}$ if $E$ is totally real. The non-minuscule types might after all be ruled out by elementary means.)

We assume that $V_{\mathrm{l}}$ is even elliptic minuscule, namely, that the derived group $G_{\mathfrak{l}}$ of $M_{\mathfrak{l}}^{\text {Zar }}$ admits at least one maximal torus acting irreducibly

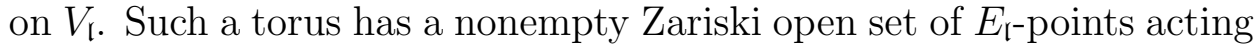
irreducibly on $V_{\mathfrak{l}}$. 
The subset of the compact analytic group $M_{\mathfrak{l}}$ consisting of those elements acting irreducibly on $V_{\mathfrak{l}}$ is a union of conjugacy classes and is open by Krasner's lemma ([9], II, Exercice 2). For elliptic minuscule $V_{\mathfrak{l}}$, this subset is nonempty whose nonzero volume in the normalized Haar measure of $M_{\mathfrak{l}}$ is by Cebotarev's density theorem the density of the set

$$
\left\{s \in S \backslash\{t\}, F_{s}^{*} \text { acts irreducibly on } V_{\mathfrak{l}}\right\},
$$

or equivalently the density of the set

$$
\left\{s \in S \backslash\{t\},\left(\operatorname{End}_{s}\left(A_{s}\right) \otimes_{\mathbf{z}} \mathbf{Q}\right) \otimes_{E} E_{\mathfrak{l}} \text { is a division algebra }\right\},
$$

which is $\leq$ the density of the set

$$
\left\{s \in S \backslash\{t\}, \operatorname{End}_{s}\left(A_{s}\right) \otimes_{\mathbf{z}} \mathbf{Q} \text { is a division algebra }\right\},
$$

or that of

$$
\left\{s \in S \backslash\{t\}, k(s) \text { is a prime field, } A_{s} \text { is simple }\right\} .
$$

So one has the following partial answer :

Theorem 1.5. Let $\ell$ be a prime number. Suppose that $E:=$ $\operatorname{End}_{t}\left(A_{t}\right) \otimes_{\mathbf{z}} \mathbf{Q}=\operatorname{End}_{\bar{t}}\left(A_{\bar{t}}\right) \otimes_{\mathbf{z}} \mathbf{Q}$ is a field, that $M_{\ell}^{\text {Zar }}$ is connected and that the monodromy representation $H^{1}\left(A_{\bar{t}}, \mathbf{Q}_{\ell}\right)$ admits an elliptic minuscule factor $V_{\mathfrak{l}}$ for a place $\mathfrak{l}$ of $E$ above $\ell$.

Then, for every prime $l, H^{1}\left(A_{\bar{t}}, \mathbf{Q}_{l}\right)$ has no multiple weights as a representation of the identity component of $M_{l}^{\mathrm{Zar}}$, and $A_{t}$ specializes to absolutely simple abelian varieties at a set of places of positive density.

To provide substance to this answer, our goal is to classify elliptic minuscule representations, namely, to solve the problem below :

Question 1.6. Let $G$ be a semi-simple algebraic group over the spectrum $\eta$ of a finite extension of $\mathbf{Q}_{\ell}$ and $\rho_{V}: G \rightarrow \mathrm{GL}(V)$ an absolutely irreducible $\eta$-linear algebraic representation with finite kernel. Does $G$ admit some maximal torus acting irreducibly on $V$ ?

One can assume $G$ to be simply connected. Let $\bar{\eta}$ be a geometric point of $\eta$. Notice that a maximal torus $\mathfrak{T}$ acts irreducibly on $V$ if and only if the weights of $V_{\bar{\eta}}$ relative to $\mathfrak{T}_{\bar{\eta}}$ are permuted transitively by $\pi_{1}(\eta, \bar{\eta})$. So if such a torus exists, all the weights have the same length, that is, $V_{\bar{\eta}}$ is minuscule.

Let $D_{\bar{\eta}}$ be the Dynkin diagram of $G_{\bar{\eta}}$ and $\rho_{D}: \pi_{1}(\eta, \bar{\eta}) \rightarrow \operatorname{Aut}\left(D_{\bar{\eta}}\right)$ the index. Let $\alpha_{i}, i=1, \cdots, r$, be the $\pi_{1}(\eta, \bar{\eta})$-orbits in $D_{\bar{\eta}}$ consisting of minuscule vertices corresponding to a minuscule representation $V=$ $V_{1} \otimes_{\eta} \cdots \otimes_{\eta} V_{r}$ of $G=G_{1} \times_{\eta} \cdots \times_{\eta} G_{r}, G_{i}$ being the simple factors. Put $D=\left(D_{\bar{\eta}}, \rho_{D}\right), \alpha_{V}=\sum \alpha_{i}$. 
Whether or not $G$ has a maximal torus acting irreducibly on $V$ depends in fact only on $\left(D, \alpha_{V}\right)(2.3,3.1)$. If $G$ admits such a torus, we call $\left(D, \alpha_{V}\right)$ an elliptic minuscule pair (2.2). The elliptic minuscule pairs with connected Dynkin diagrams are enumerated in (3.2).

Remark 1.7. Suppose that $\bar{t}$ has values in $\mathbf{C}$, that $\operatorname{End}_{\bar{t}}\left(A_{\bar{t}}\right) \otimes_{\mathbf{z}} \mathbf{Q}$ is a field and that the Mumford-Tate group of the Hodge structure on $H^{1}\left(A_{\bar{t}}^{a n}, \mathbf{Q}\right)$ is definable by absolute Hodge cycles rational over $t$ ([4], $2.11,2.9)$. It is possible that then $A_{t}$ has absolutely simple specializations at a set of places of positive density.

Acknowledgement - We owe much to M. Aschbacher for answering questions of Ying Zong. We thank J. Achter for kind correspondences. We thank especially C. Chai and S. Lu for their critical reading of the manuscript and providing comprehensive advice on writings.

\section{Elliptic Minuscule PAirs}

2.1. A Dynkin diagram is a finite set $D$ equipped with the structure of a function $l: D \rightarrow\{1,2,3\}$ ("longueurs") and of a binary relation $L$ ("liaisons") on $D$ such that $L$ is disjoint with the diagonal of $D \times D$.

Every root system has its Dynkin diagram with its connected components labeled according to types as $A, B, \cdots, G_{2}$ ([3], Chapitre VI, Théorème 3, p. 197).

Let $S$ be a scheme. An $S$-Dynkin diagram is a sheaf of sets $D$ on $S$ for the étale topology which is locally constant constructible and is equipped with the structure of a morphism $l: D \rightarrow\{1,2,3\}_{S}$ and of a sheaf of $S$-relations $L \subset D \times_{S} D, L$ locally constant constructible on $S$, such that for every geometric point $s$ of $S$ the fibre $D_{s}$ with the function $l_{s}$ and the relation $L_{s}$ is a Dynkin diagram.

For every $S$-scheme $S^{\prime}, D \times_{S} S^{\prime}$ is an $S^{\prime}$-Dynkin diagram and every descent datum on $D$ relative to $S$ for the étale topology is effective.

The monodromy representation

$$
\rho_{D, s}: \pi_{1}(S, s) \rightarrow \operatorname{Aut}\left(D_{s}, l_{s}, L_{s}\right)
$$

associated with an $S$-Dynkin diagram $D$ at a geometric point $s \rightarrow S$ is said to be the index of $D$ at $s$ (cf. [14], 2.3).

One defines $\pi_{0}(D)$ to be the quotient of $D$ by the equivalence relation generated by $L$. Notice that $D$ is a $\pi_{0}(D)$-Dynkin diagram.

Every reductive $S$-group scheme has its $S$-Dynkin diagram which is functorial with respect to isomorphisms and is compatible with every base change (SGA 3, Éxposé XXIV, 3.3). 
Given an $S$-Dynkin diagram $D$, if at every geometric point $s$ of $S$ the components of the fibre $D_{s}$ are of the types $A, B, \cdots, G_{2}$, then there is a quasi-épinglé semi-simple simply connected $S$-group scheme which has $D$ as its $S$-Dynkin diagram (SGA 3, Éxposé XXIV, Théorème 3.11).

And, for each semi-simple simply connected $S$-group scheme $G$, there exists up to unique isomorphisms a unique pair $(Q, u)$ which consists of a quasi-épinglé semi-simple simply connected $S$-group scheme $Q$ and of an "isomorphisme extérieur" $u \in \operatorname{Isom.ext}_{S}(Q, G)$ (SGA 3, Éxposé XXIV, Corollaire 3.12). The existence of $u$ enables the identification of the $S$-Dynkin diagram $D$ of $Q$ with that of $G$ and permits one to define the $S$-scheme of "isomorphismes intérieurs"

$$
\underline{\text { Isom.int }}_{S}(Q, G) \text {, }
$$

which is a left torsor under the adjoint group of $G$ and a right torsor under the adjoint group of $Q$.

Let $T \subset B$ be the canonical maximal torus and Borel subgroup of $Q, U$ the unipotent radical of $B, N$ the normalizer of $T$ in $Q$ and $W=N / T$ the Weyl group. Let

$$
\pi: X \rightarrow S
$$

denote the $S$-scheme $Q / B$, which is projective smooth with geometrically connected fibres over $S$.

Suppose that

$$
\omega: T \rightarrow \mathbf{G}_{m, S}
$$

is a weight of $Q$ with respect to $T$ that is dominant relative to the notion of positivity defined by $B$. Let

$$
\omega_{B}: B \rightarrow B / U=T \stackrel{\omega}{\longrightarrow} \mathbf{G}_{m, S}
$$

be the composition. This character $\omega_{B}$, when twisted by the $B_{X}$-torsor

$$
Q \rightarrow Q / B=X
$$

provides a $\mathbf{G}_{m, X}$-torsor

$$
Q \stackrel{B_{X}}{\wedge} \mathbf{G}_{m, X}
$$

and an invertible $\mathcal{O}_{X}$-module

$$
L_{\omega}=Q \stackrel{B_{X}}{\wedge} \mathbf{G}_{m, X} \stackrel{\mathbf{G}_{m, X}}{\wedge} \mathcal{O}_{X}
$$

Recall that $E_{\omega}=\pi_{*} L_{\omega}$ is a representation of $Q$ on a locally free $\mathcal{O}_{S^{-}}$ module of finite rank whose formation is compatible with every base change $S^{\prime} \rightarrow S$. And when $S$ is the spectrum of an algebraically closed field of characteristic zero, $E_{\omega}$ is irreducible with highest weight $\omega$. 
In particular, to each section $\alpha \in D(S)$ of the $S$-Dynkin diagram $D$, there corresponds a fundamental representation $E_{\alpha}$ of $Q$ of fundamental weight $\omega_{\alpha}$.

We say that a section $\alpha \in D(S)$ is minuscule if the Weyl orbit

$$
W \omega_{\alpha} \subset \underline{\operatorname{Hom}}_{S}\left(T, \mathbf{G}_{m, S}\right)
$$

is the sheaf of weights of $E_{\alpha}$ relative to $T$.

More generally, $\alpha=\sum_{i=1}^{r} \alpha_{i}, \alpha_{i} \in D(S)$, is said to be minuscule if each $\alpha_{i}$ is minuscule and if, for every geometric point $s$ of $S, \alpha_{i, s}$ lie in distinct components of $D_{s}$. Let $W \omega_{\alpha}:=W \omega_{\alpha_{1}} \times_{S} \cdots \times_{S} W \omega_{\alpha_{r}}$.

Definition 2.2. Suppose that $S$ is connected and that $\alpha=\sum_{i=1}^{r} \alpha_{i}$ is minuscule. The pair $(D, \alpha)$ is said to be an elliptic minuscule pair or simply elliptic if there exists a $W$-torsor $x$ on $S$ such that

$$
x \stackrel{W}{\wedge} W \omega_{\alpha}
$$

is a connected object in the Galois category of locally constant constructible sheaves on $S$, that is, if at some geometric point $s$ of $S$ the image of the monodromy representation

$$
\rho_{x, s}: \pi_{1}(S, s) \rightarrow \operatorname{Aut}\left(\left(x \stackrel{W}{\wedge} W \omega_{\alpha}\right)_{s}\right)
$$

acts transitively on the fibre $\left(x \wedge \text { } \wedge W \omega_{\alpha}\right)_{s}$. Every such $W$-torsor $x$ is said to be elliptic for $(D, \alpha)$.

One has the following result :

Theorem 2.3. Let $\eta$ be the spectrum of a complete discretely valued field of characteristic zero with finite residue field. Let $G$ be a semi-simple algebraic group over $\eta$ with Dynkin diagram $D$ and let $\rho_{V}: G \rightarrow \mathrm{GL}(V)$ be an absolutely irreducible $\eta$-linear algebraic representation with finite kernel.

Then there exists a maximal torus of $G$ acting irreducibly on $V$ if and only if $V$ is minuscule and $(D, \alpha)$ is elliptic, $\alpha$ being the minuscule section corresponding to $V$.

For its proof, we may and do assume $G$ to be simply connected.

Observe that if $G$ admits a maximal torus $\mathfrak{T}$ which acts irreducibly on $V$, then the weights of $V_{\bar{\eta}}$ relative to $\mathfrak{T}_{\bar{\eta}}$ are permuted transitively by $\pi_{1}(\eta, \bar{\eta})$. A priori, all these weights have the same length, and so $V$ is minuscule ([3], Chapitre VIII, §7, Proposition 6, p. 127).

In the following we suppose that $V$ is minuscule. Let $\alpha=\sum \alpha_{i}$ denote its corresponding minuscule section of $D$. 
Lemma 2.4. For each anisotropic maximal torus $\mathfrak{T}$ of $G$, if $\mathfrak{T}^{\mathrm{ad}}$ denotes its image in the adjoint group $G^{\text {ad }}$, the canonical map

$$
H^{1}\left(\eta, \mathfrak{T}^{\mathrm{ad}}\right) \rightarrow H^{1}\left(\eta, G^{\mathrm{ad}}\right)
$$

is surjective and $H^{2}(\eta, \mathfrak{T})=0$.

Proof. Notice that $H^{1}(\eta, G)=0$, as $G$ is by assumption simply connected (Kneser). Let $Z$ be the center of $G$. The central extension

$$
1 \rightarrow Z \rightarrow G \rightarrow G^{\text {ad }} \rightarrow 1
$$

induces the cohomology sequence

$$
H^{1}(\eta, G) \rightarrow H^{1}\left(\eta, G^{\mathrm{ad}}\right) \stackrel{\partial}{\longrightarrow} H^{2}(\eta, Z)
$$

from which it follows that

$$
\partial: H^{1}\left(\eta, G^{\text {ad }}\right) \rightarrow H^{2}(\eta, Z)
$$

is injective. To show that

$$
H^{1}\left(\eta, \mathfrak{T}^{\mathrm{ad}}\right) \rightarrow H^{1}\left(\eta, G^{\mathrm{ad}}\right)
$$

is surjective, it suffices to show that the composition

$$
\delta: H^{1}\left(\eta, \mathfrak{T}^{\mathrm{ad}}\right) \rightarrow H^{1}\left(\eta, G^{\mathrm{ad}}\right) \stackrel{\partial}{\hookrightarrow} H^{2}(\eta, Z)
$$

is surjective. The map

$$
\delta: H^{1}\left(\eta, \mathfrak{T}^{\mathrm{ad}}\right) \rightarrow H^{2}(\eta, Z)
$$

is a coboundary map induced by the central extension

$$
1 \rightarrow Z \rightarrow \mathfrak{T} \rightarrow \mathfrak{T}^{\text {ad }} \rightarrow 1
$$

and the cohomology sequence

$$
H^{1}\left(\eta, \mathfrak{T}^{\mathrm{ad}}\right) \stackrel{\delta}{\longrightarrow} H^{2}(\eta, Z) \rightarrow H^{2}(\eta, \mathfrak{T})
$$

implies that

$$
\delta: H^{1}\left(\eta, \mathfrak{T}^{\mathrm{ad}}\right) \rightarrow H^{2}(\eta, Z)
$$

is surjective if $H^{2}(\eta, \mathfrak{T})=0$.

So it remains to show that $H^{2}(\eta, \mathfrak{T})=0$. Since the Yoneda pairing

$$
\operatorname{Hom}_{\eta}\left(\mathfrak{T}, \mathbf{G}_{m}\right) \times H^{2}(\eta, \mathfrak{T}) \rightarrow H^{2}\left(\eta, \mathbf{G}_{m}\right)=\operatorname{Br}(\eta) \stackrel{\Im}{\mathbf{Q}} / \mathbf{Z}
$$

is non-degenerate (Nakayama-Tate), it suffices to show that

$$
\operatorname{Hom}_{\eta}\left(\mathfrak{T}, \mathbf{G}_{m}\right)=0 .
$$

But this latter is precisely the condition that $\mathfrak{T}$ is anisotropic. 
Let the quasi-épinglé semi-simple simply connected $\eta$-group scheme $Q$, the "isomorphisme extérieur" $u \in \operatorname{Isom}_{\operatorname{ext}}(Q, G)$ and the bitorsor $\underline{\text { Isom.int }}_{\eta}(Q, G)$ be as in (2.1).

Let $T \subset B$ be the canonical maximal torus and Borel subgroup of $Q, N$ the normalizer of $T$ in $Q, W=N / T, C$ the center of $Q$ and $T^{\text {ad }}$ (resp. $N^{\text {ad }}$ ) the image of $T$ (resp. $N$ ) in the adjoint group $Q^{\text {ad }}$.

Let $E_{\alpha}=\otimes E_{\alpha_{i}}$ be the minuscule representation of $Q$ of fundamental weight $\omega_{\alpha}$.

Lemma 2.5. 1) The $Q^{\mathrm{ad}}(\eta)$-conjugacy classes of maximal tori of $Q$ are in bijective correspondence with the elements of $H^{1}(\eta, N)$.

2) The map $H^{1}(\eta, N) \rightarrow H^{1}(\eta, W)$ is injective whose image contains those isomorphism classes of $W$-torsors $x$ on $\eta$ such that $x \stackrel{W}{\wedge} T$ is anisotropic.

Proof. 1) The set $(Q / N)(\eta)$ classifies the maximal tori of $Q$ because locally on $\eta$ for the étale topology they are all conjugate to $T$ by sections of $Q$.

The exact sequence of pointed sets

$$
Q^{\mathrm{ad}}(\eta) \rightarrow(Q / N)(\eta) \rightarrow H^{1}\left(\eta, N^{\mathrm{ad}}\right) \rightarrow H^{1}\left(\eta, Q^{\mathrm{ad}}\right)
$$

shows that the $Q^{\text {ad }}(\eta)$-orbits in $(Q / N)(\eta)$ are in one-to-one correspondence with the elements of the kernel of the map

$$
H^{1}\left(\eta, N^{\mathrm{ad}}\right) \rightarrow H^{1}\left(\eta, Q^{\mathrm{ad}}\right) .
$$

Observe that in the cohomology sequence

$$
H^{1}(\eta, Q) \rightarrow H^{1}\left(\eta, Q^{\mathrm{ad}}\right) \stackrel{\partial}{\longrightarrow} H^{2}(\eta, C)
$$

induced by the central extension

$$
1 \rightarrow C \rightarrow Q \rightarrow Q^{\mathrm{ad}} \rightarrow 1
$$

the map

$$
\partial: H^{1}\left(\eta, Q^{\mathrm{ad}}\right) \rightarrow H^{2}(\eta, C)
$$

is injective since

$$
H^{1}(\eta, Q)=0
$$

$Q$ being simply connected.

Hence, the kernel of the map

$$
H^{1}\left(\eta, N^{\mathrm{ad}}\right) \rightarrow H^{1}\left(\eta, Q^{\mathrm{ad}}\right)
$$

is equal to the kernel of the composition

$$
\delta: H^{1}\left(\eta, N^{\mathrm{ad}}\right) \rightarrow H^{1}\left(\eta, Q^{\mathrm{ad}}\right) \stackrel{\partial}{\hookrightarrow} H^{2}(\eta, C) .
$$


This

$$
\delta: H^{1}\left(\eta, N^{\mathrm{ad}}\right) \rightarrow H^{2}(\eta, C)
$$

is a coboundary map induced by the central extension

$$
1 \rightarrow C \rightarrow N \rightarrow N^{\text {ad }} \rightarrow 1
$$

From the exact sequence

$$
H^{1}(\eta, C) \rightarrow H^{1}(\eta, N) \rightarrow H^{1}\left(\eta, N^{\mathrm{ad}}\right) \stackrel{\delta}{\longrightarrow} H^{2}(\eta, C),
$$

one finds that $H^{1}(\eta, N)$ is mapped onto $\operatorname{Ker}(\delta)$ by

$$
H^{1}(\eta, N) \rightarrow H^{1}\left(\eta, N^{\mathrm{ad}}\right) .
$$

To conclude that $H^{1}(\eta, N)$ is isomorphic to this image, it suffices to show that the map

$$
H^{1}(\eta, C) \rightarrow H^{1}(\eta, N)
$$

is 0 or, by the factorization

$$
H^{1}(\eta, C) \rightarrow H^{1}(\eta, T) \rightarrow H^{1}(\eta, N),
$$

that

$$
H^{1}(\eta, T)=0
$$

This latter vanishing follows from the identity

$$
H^{1}(\eta, T)=H^{1}\left(D, \mathbf{G}_{m}\right)
$$

(SGA 3, Éxposé XXIV, Corollaire 3.14) and by Satz 90 :

$$
H^{1}\left(D, \mathbf{G}_{m}\right)=0
$$

the Dynkin diagram $D$ being representable by a finite étale $\eta$-scheme.

2) That

$$
H^{1}(\eta, N) \rightarrow H^{1}(\eta, W)
$$

is injective results from the cohomology sequence

$$
H^{1}(\eta, T) \rightarrow H^{1}(\eta, N) \rightarrow H^{1}(\eta, W)
$$

and by $H^{1}(\eta, T)=0$.

The class of a $W$-torsor $x$ on $\eta$ lies in the image of the map

$$
H^{1}(\eta, N) \rightarrow H^{1}(\eta, W)
$$

if and only if an obstruction

$$
o(x) \in H^{2}(\eta, x \stackrel{W}{\wedge} T)
$$

vanishes.

When $x \wedge T$ is anisotropic, one has in fact $H^{2}(\eta, x \wedge T)=0(2.4)$. 
Lemma 2.6. If a torus of $G$ acts irreducibly on $V$, it is anisotropic.

Proof. A torus is anisotropic if and only if it has no diagonalizable sub-torus other than 1.

Recall that the kernel of the representation

$$
\rho_{V}: G \rightarrow \mathrm{GL}(V)
$$

is finite. And $\operatorname{det}\left(\rho_{V}\right)=1$, as $G$ is semi-simple.

Suppose that a certain torus of $G$ acts irreducibly on $V$. If a $\mathbf{G}_{m}$ were in this torus, it would act on $V$ by a character $z \mapsto z^{n}$ for some integer $n$ and thus on $\operatorname{det}(V)$ by the character $z \mapsto z^{n d}$, where $d=\operatorname{dim}(V)$. So $n d=0$, i.e., $n=0$ and $\mathbf{G}_{m}$ was contained in $\operatorname{Ker}\left(\rho_{V}\right)$.

Lemma 2.7. The group $G$ has a maximal torus acting irreducibly on $V$ if and only if the group $Q$ has a maximal torus acting irreducibly on $E_{\alpha}$.

Proof. Suppose that a maximal torus $\mathfrak{T}$ of $G$ acts irreducibly on $V$. By (2.6), $\mathfrak{T}$ is anisotropic. And by (2.4), the map

$$
H^{1}\left(\eta, \mathfrak{T}^{\mathrm{ad}}\right) \rightarrow H^{1}\left(\eta, G^{\mathrm{ad}}\right)
$$

is surjective. The $G^{\text {ad }}$-torsor

$$
\underline{\text { Isom.int }}_{\eta}(Q, G)
$$

is in particular the image of a $\mathfrak{T}^{\text {ad }}$-torsor, which means (SGA 3, Éxposé XXIV, Proposition 2.11) that $\mathfrak{T}$ imbeds into $Q$ as a maximal torus and the scheme

$$
\mathfrak{I}=\underline{\text { Isom.int }}_{\eta}(Q, G ; \operatorname{Id} \text { on } \mathfrak{T})
$$

of "isomorphismes intérieurs" from $Q$ to $G$ that induce the identity automorphism on $\mathfrak{T}$ is nonempty.

Let $\bar{\eta}$ be a geometric point of $\eta$. The choice of a section $\iota \in \mathfrak{I}(\bar{\eta})$ identifies the sheaves of weights of $V$ and of $E_{\alpha}$ relative to $\mathfrak{T}$. So $E_{\alpha}$ is isomorphic to $V$ as a $\mathfrak{T}$-module. So $\mathfrak{T}$ acts irreducibly on $E_{\alpha}$.

The other direction is proven similarly.

\subsection{Proof of Theorem 2.3.}

By (2.7) it suffices to show that $(D, \alpha)$ is elliptic if and only if $Q$ has some maximal torus acting irreducibly on $E_{\alpha}$.

Suppose first that $Q$ admits a maximal torus acting irreducibly on $E_{\alpha}$. 
This torus has then the form $z \stackrel{N}{\wedge} T$ for an $N$-torsor $z$ (2.5). Relative to this torus the sheaf of weights of $E_{\alpha}$ is

$$
z \stackrel{N}{\wedge} W \omega_{\alpha} \subset z \stackrel{N}{\wedge} \underline{\operatorname{Hom}}_{\eta}\left(T, \mathbf{G}_{m}\right)
$$

The condition that $z \stackrel{N}{\wedge} T$ acts irreducibly on $E_{\alpha}$ is equivalent to the condition that $z \stackrel{N}{\wedge} W \omega_{\alpha}$ is a connected object in the Galois category of locally constant constructible sheaves on $\eta$. So $z \stackrel{N}{\wedge} W$ is a $W$-torsor elliptic for $(D, \alpha)$.

Suppose next that $(D, \alpha)$ is elliptic and that $x$ is a $W$-torsor elliptic for $(D, \alpha)$.

Let $\rho: Q \rightarrow \mathrm{GL}\left(E_{\alpha}\right)$ denote the minuscule representation corresponding to $\alpha$ and let $\rho_{T}$ be its restriction to $T$.

One has that $\operatorname{Ker}\left(\rho_{T}\right)$ is finite and that $\operatorname{det}\left(\rho_{T}\right)=1$. The torsor $x$ twists $\rho_{T}$ to a representation of $x \stackrel{W}{\wedge} T$,

$$
\rho_{x, T}: x \stackrel{W}{\wedge} T \rightarrow \operatorname{GL}\left(E_{\alpha}\right),
$$

which has $x \stackrel{W}{\wedge} W \omega_{\alpha}$ as its sheaf of weights. In particular, $\rho_{x, T}$ is irreducible. Moreover, being a twist of $\rho_{T}, \rho_{x, T}$ has finite kernel and determinant 1. As in (2.6), $x \stackrel{W}{\wedge} T$ is anisotropic. Thus it can be imbedded into $Q$ (2.5). So $x \stackrel{W}{\wedge} T$ is a sought-after maximal torus of $Q$ acting irreducibly on $E_{\alpha}$.

\section{Simple Elliptic PAIRS}

Let $S$ be a scheme. Recall that an $S$-Dynkin diagram $D$ is also a $\pi_{0}(D)$-Dynkin diagram, where $\pi_{0}(D)$ is the finite étale $S$-scheme, the quotient of $D$ by the $S$-equivalence relation generated by the $S$-binary relation $L$ ("liaisons") (2.1). The fiber $D \times_{\pi_{0}(D)} z$ is a connected Dynkin diagram for every geometric point $z$ of $\pi_{0}(D)$.

Suppose that $S$ is connected. Let $(D, \alpha)$ be as in (2.2). Suppose that $\pi_{0}(D)=S$. Then in the notations of Bourbaki-Tits ([3], Chapitre VI, Planches I-IX, p. 250-275, and [14], p. 54-61), if $D$ is non-constant, $(D, \alpha)$ can only be $\left({ }^{2} A_{n}, \alpha_{\frac{n+1}{2}}\right), n$ odd $\geq 3$, or $\left({ }^{2} D_{n}, \alpha_{1}\right), n \geq 5$, or $\left({ }^{2} D_{4}, \alpha_{i}\right), i=1,3,4$.

Let $s$ be a geometric point of $S$. We write down the condition that $(D, \alpha)$ be elliptic. 
Lemma 3.1. 1) $\left(A_{n}, \alpha_{r}\right), r \in[1, n]$, is elliptic if and only if there is a monodromy representation in the symmetric group of $n+1$ letters

$$
\rho: \pi_{1}(S, s) \rightarrow \mathfrak{S}_{n+1}
$$

whose image permutes transitively the subsets of $\{1, \cdots, n+1\}$ of cardinality $r$.

2) $\left(B_{n}, \alpha_{n}\right)$ is elliptic if and only if there is a representation

$$
\rho: \pi_{1}(S, s) \rightarrow \mathrm{GL}_{n}(\mathbf{Z})
$$

whose image lies in the group generated by the diagonal matrices and monomial matrices and acts transitively on the set

$$
\left\{ \pm e_{1} \pm \cdots \pm e_{n}\right\}
$$

where $e_{1}, \cdots, e_{n}$ denote the standard basis of $\mathbf{Z}^{n}$.

3) $\left(C_{n}, \alpha_{1}\right)$ is elliptic if and only if there is a representation

$$
\rho: \pi_{1}(S, s) \rightarrow \mathrm{GL}_{n}(\mathbf{Z})
$$

whose image lies in the group generated by the diagonal matrices and monomial matrices and acts transitively on the set

$$
\left\{e_{1}, \cdots, e_{n},-e_{1}, \cdots,-e_{n}\right\},
$$

where $e_{1}, \cdots, e_{n}$ denote the standard basis of $\mathbf{Z}^{n}$.

4) $\left(D_{n}, \alpha_{1}\right)$ is elliptic if and only if there is a representation

$$
\rho: \pi_{1}(S, s) \rightarrow \mathrm{GL}_{n}(\mathbf{Z})
$$

whose image lies in the group generated by the diagonal matrices of determinant 1 and monomial matrices and acts transitively on the set

$$
\left\{e_{1}, \cdots, e_{n},-e_{1}, \cdots,-e_{n}\right\},
$$

where $e_{1}, \cdots, e_{n}$ denote the standard basis of $\mathbf{Z}^{n}$.

5) $\left(D_{n}, \alpha_{n-1}\right)$ (resp. $\left.\left(D_{n}, \alpha_{n}\right)\right)$ is elliptic if and only if there is a representation

$$
\rho: \pi_{1}(S, s) \rightarrow \mathrm{GL}_{n}(\mathbf{Z})
$$

whose image lies in the group generated by the diagonal matrices of determinant 1 and monomial matrices and permutes transitively the vectors

$$
s_{1} e_{1}+\cdots+s_{n} e_{n}
$$

where $s_{i} \in\{1,-1\}, s_{1} \cdots s_{n}=-1$ (resp. $s_{1} \cdots s_{n}=1$ ) and $e_{1}, \cdots, e_{n}$ denote the standard basis of $\mathbf{Z}^{n}$.

6) $\left(E_{6}, \alpha_{i}\right), i=1,6$, are elliptic if and only if there is a representation

$$
\rho: \pi_{1}(S, s) \rightarrow \mathrm{O}\left(\mathbf{F}_{2}^{6}, q\right)
$$


whose image permutes transitively the nonzero q-singular vectors in $\mathbf{F}_{2}^{6}$, where $q$ is the quadratic form such that

$$
q\left(e_{i}\right)=q\left(f_{j}\right)=1, q\left(e_{i}+e_{j}\right)=q\left(f_{i}+f_{j}\right)=0, q\left(e_{i}+f_{j}\right)=\delta_{i j},
$$

where $e_{i}, f_{j}, 1 \leq i, j \leq 3$, are a basis of $\mathbf{F}_{2}^{6}$ and where $\delta_{i j}=1$, if $i=j$, and $\delta_{i j}=0$, if $i \neq j$.

7) $\left(E_{7}, \alpha_{7}\right)$ is elliptic if and only if there is a representation

$$
\rho: \pi_{1}(S, s) \rightarrow\{1,-1\} \times \operatorname{Sp}_{6}\left(\mathbf{F}_{2}\right)
$$

whose image acts transitively on $\{1,-1\} \times\left(\operatorname{Sp}_{6}\left(\mathbf{F}_{2}\right) / \mathrm{O}(q)\right)$, q being the quadratic form on $\mathbf{F}_{2}^{6}$ such that

$$
q\left(e_{i}\right)=q\left(f_{j}\right)=1, q\left(e_{i}+e_{j}\right)=q\left(f_{i}+f_{j}\right)=0, q\left(e_{i}+f_{j}\right)=\delta_{i j},
$$

where $e_{i}, f_{j}$ are the standard symplectic base of $\mathbf{F}_{2}^{6}$ and where $\delta_{i j}=1$, if $i=j$, and $\delta_{i j}=0$, if $i \neq j$.

8) $\left({ }^{2} A_{n}, \alpha_{\frac{n+1}{2}}\right), n$ odd $\geq 3$, is elliptic if and only if there is a representation

$$
\rho=\left(\rho_{1}, \rho_{2}\right): \pi_{1}(S, s) \rightarrow\{1,-1\} \times \mathfrak{S}_{n+1}
$$

whose image permutes transitively the subsets of $\{1, \cdots, n+1\}$ of cardinality $(n+1) / 2$ and whose component $\rho_{1}$ is the index of ${ }^{2} A_{n}$. Here $-1: Y \mapsto\{1, \cdots, n+1\} \backslash Y$, for any $Y \subset\{1, \cdots, n+1\}$ of cardinality $(n+1) / 2$.

9) $\left({ }^{2} D_{n}, \alpha_{1}\right), n \geq 5$, or $\left({ }^{2} D_{n}, \alpha_{i}\right), n=4, i=1,3,4$, are elliptic if and only if there is a representation

$$
\rho: \pi_{1}(S, s) \rightarrow \mathrm{GL}_{n}(\mathbf{Z})
$$

whose image lies in the group $\mathfrak{W}$ generated by the diagonal matrices and monomial matrices and acts transitively on the set $\left\{ \pm e_{1}, \cdots, \pm e_{n}\right\}$ and which when composed with the projection $\mathfrak{W} \rightarrow \mathfrak{W} / \mathfrak{W}_{1}=\{1,-1\}$ induces the index of ${ }^{2} D_{n}$ :

$$
\rho^{2} D_{n}: \pi_{1}(S, s) \stackrel{\rho}{\longrightarrow} \mathfrak{W} \rightarrow \mathfrak{W} / \mathfrak{W}_{1}=\{1,-1\},
$$

where $\mathfrak{W}_{1}$ is the subgroup of $\mathfrak{W}$ generated by the diagonal matrices of determinant 1 and monomial matrices and where $e_{1}, \cdots, e_{n}$ denote the standard basis of $\mathbf{Z}^{n}$.

Proof. Let $Q$ be a quasi-épinglé semi-simple simply connected $S$-group scheme which has $D$ as its $S$-Dynkin diagram (2.1). Let $T$ be the canonical maximal torus of $Q$. Let $R$ (resp. $W$ ) be the root system (resp. Weyl group) of $Q$ relative to $T$. One has the following canonical exact sequence of sheaves of $S$-groups for the étale topology :

$$
1 \rightarrow W \rightarrow \text { Aut }_{S}(R) \rightarrow \text { Aut }_{S}(D) \rightarrow 1 .
$$


This exact sequence induces the cohomology sequence :

$$
H^{1}(S, W) \rightarrow H^{1}\left(S, \underline{\operatorname{Aut}}_{S}(R)\right) \rightarrow H^{1}\left(S, \underline{\operatorname{Aut}}_{S}(D)\right),
$$

by which one concludes that

An $S$-form of $R, R_{1}$, is isomorphic to $x \stackrel{W}{\wedge} R$ for some $W$-torsor $x$ if and only if $R_{1}$ has its Dynkin diagram isomorphic to $D$.

When a geometric point $s$ of the connected scheme $S$ is given, the following two conditions are equivalent :

- $R_{1}$ has $D$ as its $S$-Dynkin diagram.

- the composition

$$
\pi_{1}(S, s) \stackrel{\rho_{R_{1}, s}}{\longrightarrow} \operatorname{Aut}\left(R_{s}\right) \rightarrow \operatorname{Aut}\left(D_{s}\right)
$$

is the index of $D$ at $s$, where $\rho_{R_{1}, s}$ denotes the monodromy representation associated with $R_{1}$ at $s$.

Let $x$ be a $W$-torsor and $R^{x}:=x \wedge$ $R$. Observe that the monodromy $\operatorname{Im}\left(\rho_{R^{x}, s}\right)$ at $s$ associated with every such form $R^{x}$ normalizes the weights $W_{s} \omega_{\alpha}$. The following two conditions are equivalent :

- $x \stackrel{W}{\wedge} W \omega_{\alpha}$ is a connected object in the Galois category of locally constant constructible sheaves on $S$.

- the monodromy $\operatorname{Im}\left(\rho_{R^{x}, s}\right)$ acts transitively on the weights $W_{s} \omega_{\alpha}$.

In brief, $(D, \alpha)$ is elliptic if and only if

There is a representation

$$
\rho: \pi_{1}(S, s) \rightarrow \operatorname{Aut}\left(R_{s}\right)
$$

which satisfies the following two properties :

- When composed with the projection $\operatorname{Aut}\left(R_{s}\right) \rightarrow \operatorname{Aut}\left(D_{s}\right)$ it induces the index of $D$ at $s$ :

$$
\rho_{D}: \pi_{1}(S, s) \stackrel{\rho}{\longrightarrow} \operatorname{Aut}\left(R_{s}\right) \rightarrow \operatorname{Aut}\left(D_{s}\right) .
$$

- The image of $\rho$ acts transitively on $W_{s} \omega_{\alpha}$.

If $D$ is constant, then $W$ and $R$ are constant and the class of a $W$ torsor "is" a $W$-conjugacy class of monodromy representations in $W$. This criterion simplifies then to

There is a representation

$$
\rho: \pi_{1}(S, s) \rightarrow W
$$

whose image acts transitively on the weights $W \omega_{\alpha}$.

For type $\left(A_{n}, \alpha_{r}\right)$, this says that 
There is a representation

$$
\rho: \pi_{1}(S, s) \rightarrow \mathfrak{S}_{n+1}
$$

whose image permutes transitively the subsets of $\{1, \cdots, n+1\}$ of cardinality $r$.

Indeed, in this case,

- the Weyl group "is" the symmetric group $\mathfrak{S}_{n+1}$ of $n+1$ letters.

- the Weyl orbit $W \omega_{r}$ of the minuscule weight $\omega_{r}$ "is" the collection of subsets of $\{1, \cdots, n+1\}$ of cardinality $r$ equipped with its canonical permutation action by $\mathfrak{S}_{n+1}$.

One proceeds similarly for other types provided given a description of $\operatorname{Aut}(R)$, of the Weyl group $W$, of the minuscule vertex $\alpha$ and of the weights $W \omega_{\alpha}$.

These for $\left(B_{n}, \alpha_{n}\right),\left(C_{n}, \alpha_{1}\right),\left(D_{n}, \alpha_{i}\right), i=1, n-1, n,\left(E_{6}, \alpha_{i}\right), i=1,6$, $\left({ }^{2} A_{n}, \alpha_{\frac{n+1}{2}}\right),\left({ }^{2} D_{n}, \alpha_{1}\right),\left({ }^{2} D_{4}, \alpha_{i}\right), i=1,3,4$ follow from Bourbaki [3], Chapitre VI, Planches and Chapitre VI, $n^{\circ} 4$, Exercice 2.

For $\left(E_{7}, \alpha_{7}\right)$, one can almost quote Bourbaki [3], Chapitre VI, $n^{o} 4$, Exercices $3+2$ :

Let $Q\left(E_{7}\right)$ be the root lattice and $P\left(E_{7}\right)$ the weight lattice of a root system of type $E_{7}$. Then $2 P\left(E_{7}\right) \subset Q\left(E_{7}\right)$ and the quotient $E=$ $Q\left(E_{7}\right) / 2 P\left(E_{7}\right)$ is a 6 -dimensional $\mathbf{F}_{2}$-vector space on which the Killing form (, ) induces a symplectic form. The Weyl group $W\left(E_{7}\right)$ acts on $E$ preserving $($,$) and it maps onto \operatorname{Sp}(E)$ with kernel $\{1,-1\}$ of order 2 , loc.cit. The central extension

$$
1 \rightarrow\{1,-1\} \rightarrow W\left(E_{7}\right) \rightarrow \operatorname{Sp}(E) \rightarrow 1
$$

splits. Let $\left\{\alpha_{1}, \cdots, \alpha_{7}\right\}$ be a base of $E_{7}$ so that $\left\{\alpha_{1}, \cdots, \alpha_{6}\right\}$ generates a root system of type $E_{6}$. Observe that the roots of this sub-system

$$
\begin{gathered}
e_{1}=\alpha_{1}+\alpha_{2}+2 \alpha_{3}+2 \alpha_{4}+\alpha_{5}+\alpha_{6}, \\
e_{2}=\alpha_{1}+\alpha_{2}+\alpha_{3}+\alpha_{4}+\alpha_{5}, \\
e_{3}=\alpha_{2}+\alpha_{4}, \\
f_{1}=\alpha_{1}+\alpha_{3}+\alpha_{4}, \\
f_{2}=\alpha_{4}+\alpha_{5}+\alpha_{6}, \\
f_{3}=\alpha_{3}+\alpha_{4}+\alpha_{5}
\end{gathered}
$$

satisfy the orthogonality relations

$$
\left(e_{i}, e_{j}\right)=2 \delta_{i j}, \quad\left(f_{i}, f_{j}\right)=2 \delta_{i j}, \quad\left(e_{i}, f_{j}\right)=\delta_{i j}
$$

and that their images in $E$ form a symplectic base. In particular,

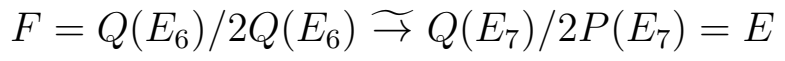


is a bijection, where $Q\left(E_{6}\right)$ denotes the root lattice of $E_{6}$.

When $F$ is equipped with the quadratic form $q=\frac{1}{2}(),, W\left(E_{6}\right)$ is identified with $\mathrm{O}(q)$ (loc.cit.). Hence,

$$
W\left(E_{7}\right) \omega_{7}=W\left(E_{7}\right) / W\left(E_{6}\right)=\{1,-1\} \times(\operatorname{Sp}(E) / \mathrm{O}(q)) .
$$

It is evident that ellipticity is a nonempty condition only when the base scheme has a rather "small" fundamental group.

Theorem 3.2. Let $S$ be the spectra of a complete discrete valuation ring, $\eta$ (resp. s) its generic (resp. closed) point and $\bar{\eta}$ a geometric generic point. Suppose that $k(\eta)$ is of characteristic zero and that $k(s)$ is finite of characteristic $\ell$.

Then the elliptic minuscule pairs $(D, \alpha)$ over $\eta$ such that $D_{\bar{\eta}}$ is a connected Dynkin diagram are enumerated in the following list :

A) $\left(A_{n}, \alpha_{1}\right),\left(A_{n}, \alpha_{n}\right), n \geq 1$, every prime $\ell$,

$\left(A_{\ell^{d}-1}, \alpha_{2}\right),\left(A_{\ell^{d}-1}, \alpha_{\ell^{d}-2}\right)$, d an integer $\geq 1$, every prime $\ell$,

$\left(A_{p-1}, \alpha_{2}\right),\left(A_{p-1}, \alpha_{p-2}\right), p$ prime, $p \equiv 1 \bmod 4, \operatorname{Card}(k(s)) \bmod p$ generates $\mathbf{F}_{p}^{\times}$,

$\left(A_{p-1}, \alpha_{2}\right),\left(A_{p-1}, \alpha_{p-2}\right), p$ prime, $p \equiv 3 \bmod 4, \operatorname{Card}(k(s)) \bmod p$ generates a subgroup of $\mathbf{F}_{p}^{\times}$of index $\leq 2$,

$\left(A_{7}, \alpha_{3}\right),\left(A_{7}, \alpha_{5}\right), \ell=2$,

$\left(A_{31}, \alpha_{3}\right),\left(A_{31}, \alpha_{29}\right), \ell=2,5 \nmid\left[s: \mathbf{F}_{2}\right] ;$

${ }^{2}$ A) $\left({ }^{2} A_{3}, \alpha_{2}\right)$, every prime $\ell$,

$\left({ }^{2} A_{5}, \alpha_{3}\right), \ell=5$,

$\left({ }^{2} A_{5}, \alpha_{3}\right),{ }^{2} A_{5}$ ramified over $S$, $\operatorname{Card}(k(s)) \bmod 5$ generates $\mathbf{F}_{5}^{\times}$;

B) $\left(B_{3}, \alpha_{3}\right),\left(B_{4}, \alpha_{4}\right)$, every prime $\ell$,

$\left(B_{n}, \alpha_{n}\right), n \geq 5, \ell=2$

C) $\left(C_{n}, \alpha_{1}\right), n \geq 2$, every prime $\ell$;

D) $\left(D_{n}, \alpha_{1}\right), n$ odd $\geq 5, \ell=2$,

$\left(D_{n}, \alpha_{1}\right), n$ even $\geq 4$, every prime $\ell$,

$\left(D_{5}, \alpha_{4}\right),\left(D_{5}, \alpha_{5}\right)$, every prime $\ell$,

$\left(D_{n}, \alpha_{n-1}\right),\left(D_{n}, \alpha_{n}\right), n \geq 6, \ell=2$;

$\left.{ }^{2} D\right)\left({ }^{2} D_{n}, \alpha_{1}\right), n \geq 5$, every prime $\ell$;

$\left.E_{6}\right)\left(E_{6}, \alpha_{1}\right),\left(E_{6}, \alpha_{6}\right), \ell=3$,

$\left(E_{6}, \alpha_{1}\right),\left(E_{6}, \alpha_{6}\right), \operatorname{Card}(k(s)) \equiv \pm 2, \pm 4 \bmod 9 ;$ 
$\left.E_{7}\right)\left(E_{7}, \alpha_{7}\right), \ell=2$.

This list is justified in the remaining sections.

\section{TWO LEMMAS}

Let $S$ be the spectra of a complete discrete valuation ring and $\eta$ (resp. $s)$ its generic (resp. closed) point. Suppose that $k(\eta)$ is of characteristic zero and that $k(s)$ is finite of characteristic $\ell$. Let $\bar{\eta}$ (resp. $\bar{s}$ ) be the spectrum of an algebraic closure of $k(\eta)$ (resp. $k(s))$.

As $S$ is complete along $s$, the inclusion $s \hookrightarrow S$ induces a bijection

$$
\pi_{1}(s, \bar{s}) \widetilde{\rightrightarrows} \pi_{1}(S, \bar{s}) .
$$

The group $\pi_{1}(s, \bar{s})$ is isomorphic to $\widehat{\mathbf{Z}}$ with the Frobenius $F_{s}$ as its canonical generator. For each integer $N \geq 1$ there is thus up to isomorphisms a unique spectra $S_{N}$ of a discrete valuation ring such that $S_{N}$ is finite étale Galois over $S$ with cyclic Galois group of order $N$.

Let $S_{(\bar{s})}$ be the strict localization of $S$ at $\bar{s}$ and $\eta^{\text {hs }}$ the generic point of $S_{(\bar{s})}$. The open immersion $\eta \hookrightarrow S$ induces a surjection

$$
\pi_{1}(\eta, \bar{\eta}) \rightarrow \pi_{1}(S, \bar{\eta}) \simeq \pi_{1}(S, \bar{s}),
$$

whose kernel, the inertia subgroup of $\pi_{1}(\eta, \bar{\eta})$, is isomorphic to $\pi_{1}\left(\eta^{h s}, \bar{\eta}\right)$. This inertia subgroup admits a canonical surjection

$$
\pi_{1}\left(\eta^{h s}, \bar{\eta}\right) \rightarrow \prod_{p \neq \ell} \mathbf{Z}_{p}(1)
$$

which corresponds by Galois theory to the subextension of $k(\bar{\eta}) / k\left(\eta^{h s}\right)$ obtained by joining to $k\left(\eta^{h s}\right)$ all $N$-th roots of a uniformizer of $S_{(\bar{s})}$ for all integers $N$ prime to $\ell$. The kernel of this surjection, the wild inertia subgroup of $\pi_{1}(\eta, \bar{\eta})$, is a pro- $\ell$-group and normal in $\pi_{1}(\eta, \bar{\eta})$.

In particular, the group $\pi_{1}(\eta, \bar{\eta})$ is pro-solvable.

The quotient of $\pi_{1}(\eta, \bar{\eta})$ by its wild inertia subgroup is denoted by $\pi_{1}^{t}(\eta, \bar{\eta})$, which as a profinite group admits 2 generators $F, T$ and 1 single relation :

$$
F T F^{-1}=T^{q}
$$

where $q=\operatorname{Card}(k(s))$.

A monodromy representation $\pi_{1}(\eta, \bar{\eta}) \rightarrow \mathfrak{G}$ is said to be unramified (resp. tamely ramified) over $S$ if its kernel contains the inertia (resp. wild inertia) subgroup. A quotient $\mathfrak{G}$ of $\pi_{1}(\eta, \bar{\eta})$ is said to be unramified (resp. tamely ramified) over $S$ if the quotient homomorphism $\pi_{1}(\eta, \bar{\eta}) \rightarrow \mathfrak{G}$ is. 
We will apply the following two simple lemmas a few times.

Lemma 4.1. Let $N$ be an integer $\geq 1$. Let $\zeta \in \mathrm{GL}_{N}\left(\mathbf{F}_{\ell}\right)$ be such that

$$
\zeta: e_{1} \mapsto e_{2}, e_{2} \mapsto e_{3}, \cdots, e_{N} \mapsto e_{1},
$$

where $e_{1}, \cdots, e_{N}$ denote the standard basis of $\mathbf{F}_{\ell}^{N}$.

Then the semi-direct product $\langle\zeta\rangle \mathbf{F}_{\ell}^{N}$ is a quotient of $\pi_{1}(\eta, \bar{\eta})$. If $(\ell, N)=1$ and if $V$ is an irreducible $\mathbf{F}_{\ell}$-linear representation of $\langle\zeta\rangle$, then $\langle\zeta\rangle V$ is a quotient of $\pi_{1}(\eta, \bar{\eta})$.

Proof. Let $\pi \in \Gamma\left(S, \mathcal{O}_{S}\right)$ be a uniformizer. Let $S^{\prime}$ be the spectra of a discrete valuation ring such that $S^{\prime}$ is finite étale Galois over $S$ with cyclic Galois group of order $N$. Let $\eta^{\prime}$ (resp. $s^{\prime}$ ) be the generic (resp. closed) point of $S^{\prime}, \zeta$ a generator of $\operatorname{Gal}\left(S^{\prime} / S\right)$ and let $u^{\prime} \in \Gamma\left(S^{\prime}, \mathcal{O}_{S^{\prime}}\right)^{\times}$ be a unit such that the images of $u^{\prime}, \zeta\left(u^{\prime}\right), \cdots, \zeta^{N-1}\left(u^{\prime}\right)$ in $k\left(s^{\prime}\right)$ form a normal base over $k(s)$. Then

$$
\eta^{\prime}\left[x_{1}, \cdots, x_{N}\right] /\left(x_{1}^{\ell}-x_{1}-\zeta\left(u^{\prime}\right) \pi^{-1}, \cdots, x_{N}^{\ell}-x_{N}-\zeta^{N}\left(u^{\prime}\right) \pi^{-1}\right)
$$

is connected and Galois over $\eta$ with Galois group $\langle\zeta\rangle \mathbf{F}_{\ell}^{N}$. If $(\ell, N)=1$, $\langle\zeta\rangle V$ is a quotient of $\langle\zeta\rangle \mathbf{F}_{\ell}^{N}$ and hence is a quotient of $\pi_{1}(\eta, \bar{\eta})$.

Lemma 4.2. Let $p$ be a prime number different from $\ell$.

1) If the underlying group of an $\mathbf{F}_{p}$-vector space $V$ is a normal subgroup of a finite quotient $\mathfrak{G}$ of $\pi_{1}(\eta, \bar{\eta})$ such that $\mathfrak{G}$ acts irreducibly on $V$ by conjugation, then $\operatorname{dim} V=1$.

2) There is a unique group of affine linear transformations of $\mathbf{F}_{p}$ which contains all translations and which is a quotient of $\pi_{1}(\eta, \bar{\eta}) \mathrm{ram}$ ified over $S$. This group has cardinality $p N$, where $N$ is the order of the element $\operatorname{Card}(k(s)) \bmod p$ in $\mathbf{F}_{p}^{\times}$.

Proof. 1) Let $I$ (resp. $P$ ) be the image in $\mathfrak{G}$ of the inertia (resp. wild inertia) subgroup of $\pi_{1}(\eta, \bar{\eta})$. Notice that $V \cap P=1$. The intersection $V \cap I$ being normal in $\mathfrak{G}$ is a sub-Ǵ-module of $V$. As $V$ is by assumption an irreducible $\mathfrak{G}$-module, one has $V \cap I=1$ or $V$.

If $V \cap I=1$, then $V$ is isomorphic to a subgroup of $\mathfrak{G} / I$ and thus is cyclic.

If $V \cap I=V$, then $V$ is isomorphic to a subgroup of $I / P$ and thus is again cyclic.

2) Let $q=\operatorname{Card}(k(s))$. Let $t: x \mapsto x+1, \forall x \in \mathbf{F}_{p}$. For every $a \in \mathbf{F}_{p}^{\times}$, let $l_{a}: x \mapsto a x, \forall x \in \mathbf{F}_{p}$. The following relation holds :

$$
l_{a} t l_{a}^{-1}=t^{a}: x \mapsto x+a, \forall x \in \mathbf{F}_{p} .
$$


In particular, writing $N$ for the order of $q \bmod p$ as an element of $\mathbf{F}_{p}^{\times}$, the group generated by $\left\{l_{q}, t\right\}$ has order $p N$ and it is a quotient of $\pi_{1}(\eta, \bar{\eta})$ tamely ramified over $S$ :

$$
\pi_{1}^{t}(\eta, \bar{\eta}) \rightarrow\left\langle l_{q}, t\right\rangle, F \mapsto l_{q}, T \mapsto t .
$$

Suppose that another representation of $\pi_{1}(\eta, \bar{\eta})$ in the group of affine linear transformations of $\mathbf{F}_{p}$ is ramified over $S$ and has $t$ in its image $\mathfrak{G}$. Let $I$ (resp. $P$ ) be the image in $\mathfrak{G}$ of the inertia (resp. wild inertia) subgroup of $\pi_{1}(\eta, \bar{\eta})$.

In the group of affine linear transformations of $\mathbf{F}_{p}$, the subgroup of translations is its own centralizer and it intersects $P$ in 1 . So $P=1$. So $I=I / P$ is cyclic and $\neq 1$. Either $I$ contains $t$ or it intersects the group of translations in 1 . In both cases, $t$ commutes with all elements of $I$. Hence $I$ is the group of all translations.

In brief, the quotient homomorphism $\pi_{1}(\eta, \bar{\eta}) \rightarrow \mathfrak{G}$ factors through $\pi_{1}^{t}(\eta, \bar{\eta})=\langle F, T\rangle$ and it maps $T$ to a non-zero translation.

Let the image of $F(\operatorname{resp} . T)$ in $\mathfrak{G}$ be $l_{a} t^{b}\left(\operatorname{resp} . t^{c}\right)$, where $a, c \in$ $\mathbf{F}_{p}^{\times}, b \in \mathbf{F}_{p}$. The identity

$$
\left(l_{a} t^{b}\right) t^{c}\left(l_{a} t^{b}\right)^{-1}=\left(t^{c}\right)^{q}
$$

says that $a c=q c$, namely, that $a=q \bmod p$. So $\mathfrak{G}=\left\langle l_{q} t^{b}, t^{c}\right\rangle=\left\langle l_{q}, t\right\rangle$.

\section{TYPE $A$}

Let $(S, \eta, s)$, $\operatorname{char}(s)=\ell$, be as in $\S 4$.

Proposition 5.1. For every integer $n \geq 1,\left(A_{n}, \alpha_{1}\right)$ and $\left(A_{n}, \alpha_{n}\right)$ are elliptic over $\eta$.

Proof. The subgroup of $\mathfrak{S}_{n+1}$ generated by the cycle $(12 \cdots n+1)$ acts transitively on $\{1, \cdots, n+1\}$ and permutes transitively the subsets of $\{1, \cdots, n+1\}$ of cardinality $n$. As $\langle(12 \cdots n+1)\rangle=\mathbf{Z} /(n+1) \mathbf{Z}$ is a quotient of $\pi_{1}(\eta, \bar{\eta})(\S 4)$, both $\left(A_{n}, \alpha_{1}\right)$ and $\left(A_{n}, \alpha_{n}\right)$ are elliptic over $\eta$ $(3.1), 1)$.

Lemma 5.2. Let $X$ be a finite set of cardinality $q \geq 4$. Let $r$ be an integer such that $2 \leq r \leq q / 2$. Suppose that the subsets of $X$ of cardinality $r$ are permuted transitively by a solvable subgroup $\mathfrak{G}$ of $\operatorname{Aut}(X)$. Then $r<4$. Moreover,

1) If $r=2, \mathfrak{G}$ acts 2-transitively on $X$ unless : 
$-X=\mathbf{F}_{q}, q \equiv 3 \bmod 4$ and, for some subfield $k$ of $\mathbf{F}_{q}, \mathfrak{G}$ consists of all transformations of the form :

$$
x \mapsto a^{2} \varphi(x)+b, \forall x \in \mathbf{F}_{q}
$$

where $a \in \mathbf{F}_{q}^{\times}, b \in \mathbf{F}_{q}, \varphi \in \operatorname{Gal}\left(\mathbf{F}_{q} / k\right)$.

2) If $r=3$, then $X=\mathbf{F}_{32}$ or $\mathbf{F}_{8}$. When $X=\mathbf{F}_{32}, \mathfrak{G}$ consists of all affine semi-linear transformations of $X$. When $X=\mathbf{F}_{8}, \mathfrak{G}$ consists of either all affine semi-linear transformations or only of the affine linear transformations of $X$.

Proof. That $r<4$ as well as 2) is extracted from [7], p. 402-403.

Suppose that $r=2$ and that $\mathfrak{G}$ does not act 2-transitively on $X$. By loc.cit., then $X=\mathbf{F}_{p^{d}}, p$ prime $\equiv 3 \bmod 4, d$ is odd and $\mathfrak{G}=\mathfrak{L} \mathfrak{T}$, where $\mathfrak{L} \leq \mathrm{GL}_{d}\left(\mathbf{F}_{p}\right)$ has odd order and where $\mathfrak{T}$ is the group of all translations of $X$. Observe that -1 then normalizes $\mathfrak{G}$ and that $\{1,-1\} \mathfrak{G}$ acts 2 transitively on $X$, where $-1: x \mapsto-x, \forall x \in X$. Now 1) follows by the classification of 2 -transitive solvable permutation groups.

Corollary 5.3. If $4 \leq r \leq(n+1) / 2$, then $\left(A_{n}, \alpha_{r}\right)$ and $\left(A_{n}, \alpha_{n+1-r}\right)$ are not elliptic over $\eta$. The pairs $\left(A_{n}, \alpha_{3}\right)$ and $\left(A_{n}, \alpha_{n-2}\right)$ are elliptic over $\eta$ only if $n=7$ or 31 . The pairs $\left(A_{n}, \alpha_{2}\right)$ and $\left(A_{n}, \alpha_{n-1}\right)$ are elliptic over $\eta$ only if $n=p^{d}-1, p$ prime, $d \geq 1$.

Proof. This is immediate from $(5.2)+(3.1), 1)$. Recall that the group $\pi_{1}(\eta, \bar{\eta})$ is pro-solvable $(\S 4)$.

Proposition 5.4. Let $p$ be a prime number and $d$ an integer $\geq 1$. The pairs $\left(A_{p^{d}-1}, \alpha_{2}\right)$ and $\left(A_{p^{d}-1}, \alpha_{p^{d}-2}\right)$ are elliptic over $\eta$ if $p=\ell$ and only if $p=\ell$ when $d \geq 2$.

Proof. If a solvable subgroup of $\mathfrak{S}_{p^{d}}$ permutes transitively the 2-point subsets of $\mathbf{F}_{p}^{d}=V$, then it is of the form $\mathfrak{G}=\mathfrak{L} \mathfrak{T}$, where $\mathfrak{L}$ is a certain subgroup of GL( $V)$ acting irreducibly on $V$ and where $\mathfrak{T}$ is the group of all translations of $V(5.2), 1)$.

If $p \neq \ell$ and if $d \geq 2, \pi_{1}(\eta, \bar{\eta})$ has no such quotient as $\left.\mathfrak{G}(4.2), 1\right)$ and hence $\left(A_{p^{d}-1}, \alpha_{2}\right)$ and $\left(A_{p^{d}-1}, \alpha_{p^{d}-2}\right)$ are not elliptic over $\left.\eta(3.1), 1\right)$.

Suppose that $p=\ell$. On $\mathbf{F}_{\ell^{d}}$ the group $\mathfrak{G}$ of all affine linear transformations acts 2-transitively. And by $(4.1) \mathfrak{G}$ is a quotient of $\pi_{1}(\eta, \bar{\eta})$. So $\left(A_{\ell^{d}-1}, \alpha_{2}\right)$ and $\left(A_{\ell^{d}-1}, \alpha_{\ell^{d}-2}\right)$ are elliptic over $\left.\eta(3.1), 1\right)$. 
Proposition 5.5. Let $p$ be an odd prime different from $\ell$.

- Case $p \equiv 1 \bmod 4:$ Then $\left(A_{p-1}, \alpha_{2}\right)$ and $\left(A_{p-1}, \alpha_{p-2}\right)$ are elliptic over $\eta$ if and only if $\operatorname{Card}(k(s)) \bmod p$ generates $\mathbf{F}_{p}^{\times}$.

- Case $p \equiv 3 \bmod 4:$ Then $\left(A_{p-1}, \alpha_{2}\right)$ and $\left(A_{p-1}, \alpha_{p-2}\right)$ are elliptic over $\eta$ if and only if $\operatorname{Card}(k(s))$ mod $p$ generates a subgroup of $\mathbf{F}_{p}^{\times}$of index $\leq 2$.

Proof. By $(3.1), 1)$ the pairs $\left(A_{p-1}, \alpha_{2}\right)$ and $\left(A_{p-1}, \alpha_{p-2}\right)$ are elliptic over $\eta$ if and only if there is a representation $\pi_{1}(\eta, \bar{\eta}) \rightarrow \mathfrak{S}_{p}$ whose image $\mathfrak{G}$ permutes transitively the 2-point subsets of $\mathbf{F}_{p}$.

By $(5.2), 1)$ and by the classification of 2-transitive solvable permutation groups of degree $p$, such $\mathfrak{G}$ can only be

- (Case $p \equiv 1 \bmod 4)$ the group of all affine linear transformations of $\mathbf{F}_{p}$.

- (Case $p \equiv 3 \bmod 4)$ either the group of all affine linear transformations of $\mathbf{F}_{p}$ or the subgroup consisting of all transformations of the form $x \mapsto a^{2} x+b, \forall x \in \mathbf{F}_{p}$, where $a \in \mathbf{F}_{p}^{\times}, b \in \mathbf{F}_{p}$.

Now by (4.2), 2) the lemma follows.

Proposition 5.6. The pairs $\left(A_{7}, \alpha_{3}\right)$ and $\left(A_{7}, \alpha_{5}\right)$ are elliptic over $\eta$ if and only if $k(s)$ is of characteristic 2.

Proof. In (5.2), 2) either of the two solvable subgroups of $\mathfrak{S}_{8}$ that permute transitively the 3-point subsets of $\mathbf{F}_{8}$ contains all translations of $\mathbf{F}_{8}$. So $\left(A_{7}, \alpha_{3}\right)$ and $\left(A_{7}, \alpha_{5}\right)$ are elliptic over $\eta$ only if $k(s)$ is of characteristic $\ell=2(3.1), 1)+(4.2), 1)$.

If $\ell=2$, the group of all affine linear transformations of $\mathbf{F}_{8}$ is a quotient of $\pi_{1}(\eta, \bar{\eta})(4.1)$ and hence $\left(A_{7}, \alpha_{3}\right)$ and $\left(A_{7}, \alpha_{5}\right)$ are elliptic over $\eta(3.1), 1)+(5.2), 2)$.

Proposition 5.7. The pairs $\left(A_{31}, \alpha_{3}\right)$ and $\left(A_{31}, \alpha_{29}\right)$ are elliptic over $\eta$ if and only if $\ell=2,5 \nmid\left[s: \mathbf{F}_{2}\right]$.

Proof. The pairs $\left(A_{31}, \alpha_{3}\right)$ and $\left(A_{31}, \alpha_{29}\right)$ are elliptic over $\eta$ if and only if $\pi_{1}(\eta, \bar{\eta})$ has as quotient the group $\mathfrak{G}$ of all affine semi-linear transformations of $\left.\mathbf{F}_{32}(3.1), 1\right)$, (5.2), 2).

By $(4.2), 1) \mathfrak{G}$ is a quotient of $\pi_{1}(\eta, \bar{\eta})$ only if $k(s)$ is of characteristic $\ell=2$.

Suppose that $\ell=2$.

Suppose that $\pi_{1}(\eta, \bar{\eta})$ has $\mathfrak{G}$ as a quotient. Then $5 \nmid\left[s: \mathbf{F}_{2}\right]$. 
Let $I$ (resp. $P$ ) be the image in $\mathfrak{G}$ of the inertia (resp. wild inertia) subgroup of $\pi_{1}(\eta, \bar{\eta})$. It is immediate that $P$ (resp. $I$ ) must consist of all translations (resp. all affine linear transformations) of $\mathbf{F}_{32}$. The subgroup of $\mathfrak{G}$ generated by the Frobenius $F: x \mapsto x^{2}$ and the scalar multiplications $l_{a}: x \mapsto a x$ is isomorphic to $\mathfrak{G} / P$. By $\left.(4.2), 2\right)$ one concludes that the element $\operatorname{Card}(k(s)) \bmod 31$ must be of order 5 in $\mathbf{F}_{31}^{\times}$. That is, $5 \nmid\left[s: \mathbf{F}_{2}\right]$, since $2 \bmod 31$ is of order 5 in $\mathbf{F}_{31}^{\times}$.

Suppose that $5 \nmid\left[s: \mathbf{F}_{2}\right]$. Then $\mathfrak{G}$ is a quotient of $\pi_{1}(\eta, \bar{\eta})$.

Let $S^{\prime}$ be the spectra of a discrete valuation ring such that $S^{\prime}$ is finite étale Galois over $S$ with cyclic Galois group of order $5(\S 4)$. Let $\eta^{\prime}$ (resp. $\left.s^{\prime}\right)$ be the generic (resp. closed) point of $S^{\prime}, \zeta \in \operatorname{Gal}\left(S^{\prime} / S\right)$ a generator, $\pi \in \Gamma\left(S, \mathcal{O}_{S}\right)$ a uniformizer and let $u^{\prime} \in \Gamma\left(S^{\prime}, \mathcal{O}_{S^{\prime}}\right)^{\times}$be a unit such that the images of $u^{\prime}, \zeta\left(u^{\prime}\right), \cdots, \zeta^{4}\left(u^{\prime}\right)$ in $k\left(s^{\prime}\right)$ form a normal base over $k(s)$. Then

$$
\eta^{\prime}\left[z, x_{1}, \cdots, x_{5}\right] /\left(z^{31}-\pi, x_{1}^{2}-1-z \zeta\left(u^{\prime}\right), \cdots, x_{5}^{2}-1-z \zeta^{5}\left(u^{\prime}\right)\right)
$$

is connected and Galois over $\eta$ with Galois group $\mathfrak{G}$.

\section{6. $\mathrm{TYPE}^{2} A$}

Proposition 6.1. Let $X$ be a finite set of even cardinality $2 d$. Let $\mathfrak{G}$ be a solvable subgroup of $\operatorname{Aut}(X)$ which permutes the subsets of $X$ of cardinality d in 2 orbits.

The following list enumerates such $(X, \mathfrak{G})$ up to equivalence :

1) $X=\{o, 1\}, \mathfrak{G}=1$.

2) $X=\{o, 1,2,3\}$, $\mathfrak{G}$ fixes $o$ and on $\{1,2,3\}$ it is either $\mathfrak{S}_{3}$ or $\mathfrak{A}_{3}$.

3) $X=\{o\} \cup \mathbf{F}_{5}, \mathfrak{G}$ fixes $o$ and on $\mathbf{F}_{5}$ it is the group of all affine linear transformations.

4) $X=\mathbf{Z} / 4 \mathbf{Z}, \mathfrak{G}$ consists of either all transformations

$$
x \mapsto a x+b, \forall x \in \mathbf{Z} / 4 \mathbf{Z}
$$

where $a \in(\mathbf{Z} / 4 \mathbf{Z})^{\times}, b \in \mathbf{Z} / 4 \mathbf{Z}$ or only of the translations

$$
x \mapsto x+b, \forall x \in \mathbf{Z} / 4 \mathbf{Z}
$$

where $b \in \mathbf{Z} / 4 \mathbf{Z}$.

5) $X=\{1, \cdots, 6\}$, either $\mathfrak{G}$ is the normalizer $\mathfrak{N}$ in $\operatorname{Aut}(X)$ of a partition $X=\{a, b, c\} \cup\left\{a^{\prime}, b^{\prime}, c^{\prime}\right\}$ or it is the subgroup of $\mathfrak{N}$ generated by $\mathfrak{A} \mathfrak{t}(\{a, b, c\}) \mathfrak{A} \mathfrak{l} \mathfrak{t}\left(\left\{a^{\prime}, b^{\prime}, c^{\prime}\right\}\right)$ and one of the following subgroups :

$-\left\langle\left(a a^{\prime}\right)\left(b b^{\prime}\right)\left(c c^{\prime}\right)\right\rangle$ 
$-\left\langle\left(a a^{\prime} b b^{\prime}\right)\left(c c^{\prime}\right)\right\rangle$

$-\left\langle\left(a a^{\prime}\right)\left(b b^{\prime}\right)\left(c c^{\prime}\right),(a b)\left(a^{\prime} b^{\prime}\right)\right\rangle$

6) $X=\{1, \cdots, 6\}$, either $\mathfrak{G}$ is the normalizer $\mathfrak{N}$ in $\operatorname{Aut}(X)$ of a partition $X=\left\{a, a^{\prime}\right\} \cup\left\{b, b^{\prime}\right\} \cup\left\{c, c^{\prime}\right\}$ or it is the subgroup of $\mathfrak{N}$ generated by $\left\{\left(a a^{\prime}\right),\left(b b^{\prime}\right),\left(c c^{\prime}\right),(a b c)\left(a^{\prime} b^{\prime} c^{\prime}\right)\right\}$.

7) $X=\mathbf{F}_{8}, \mathfrak{G}$ consists of either all affine semi-linear transformations

$$
x \mapsto a x^{2^{c}}+b, \forall x \in \mathbf{F}_{8}
$$

where $a \in \mathbf{F}_{8}^{\times}, b \in \mathbf{F}_{8}, c \in \mathbf{Z} / 3 \mathbf{Z}$ or only of the affine linear transformations

$$
x \mapsto a x+b, \forall x \in \mathbf{F}_{8}
$$

where $a \in \mathbf{F}_{8}^{\times}, b \in \mathbf{F}_{8}$.

The proof is divided into several parts : (6.2), (6.4), (6.5), (6.6).

Lemma 6.2. With the notations and assumptions of (6.1), suppose furthermore that $\mathfrak{G}$ does not act transitively on $X$.

The following list enumerates all such $(X, \mathfrak{G})$ up to equivalence:

1) $X=\{o, 1\}, \mathfrak{G}=1$.

2) $X=\{o, 1,2,3\}$, $\mathfrak{G}$ fixes $o$ and on $\{1,2,3\}$ it is either $\mathfrak{S}_{3}$ or $\mathfrak{A}_{3}$.

3) $X=\{o\} \cup \mathbf{F}_{5}, \mathfrak{G}$ fixes o and on $\mathbf{F}_{5}$ it is the group of affine linear transformations.

Proof. Choose $o \in X$ such that $O=\mathfrak{G} o$ has cardinality $\leq d=$ $\operatorname{Card}(X) / 2$. Such a point exists since by assumption $\mathfrak{G}$ does not act transitively on $X$.

Choose a subset $Y$ (resp. $Z$ ) of $X$ with $d$ elements such that $Y$ (resp. Z) contains (resp. is disjoint with) $O$. One has $g Y \supset O$ and $g Z \cap O=\emptyset, \forall g \in \mathfrak{G}$. So $\mathfrak{G} Y$ and $\mathfrak{G} Z$ are these two $\mathfrak{G}$-orbits in the collection of $d$-point subsets of $X$.

Choose a point $z \in Z$. The set $\{o\} \cup Z \backslash\{z\}$ has $d$ elements and it intersects $O$ in $\{o\}$. So $O=\{o\}$.

Now $X \backslash\{o\}$ has $2 d-1$ elements and its subsets of cardinality $d$ form a single $\mathfrak{G}$-orbit $\mathfrak{G} Z$. The following lemma applies.

Lemma 6.3. Let $X$ be a finite set of odd cardinality $2 d-1$. Let $\mathfrak{G}$ be a solvable subgroup of $\operatorname{Aut}(X)$ which permutes transitively the subsets of $X$ of cardinality $d$.

The following list enumerates such $(X, \mathfrak{G})$ up to equivalence :

1) $X=1, \mathfrak{G}=1$. 
2) $X=\{1,2,3\}, \mathfrak{G}=\mathfrak{S}_{3}$ or $\mathfrak{A}_{3}$.

3) $X=\mathbf{F}_{5}, \mathfrak{G}$ consists of all affine linear transformations

$$
x \mapsto a x+b, \forall x \in \mathbf{F}_{5}
$$

where $a \in \mathbf{F}_{5}^{\times}, b \in \mathbf{F}_{5}$.

Proof. If $d=1$, then $X=1$ and $\mathfrak{G}=1$, hence 1 ).

Suppose $d>1$. Notice that

- The group $\mathfrak{G}$ acts transitively on $X$ :

Otherwise, some $\mathfrak{G}$-orbit in $X$, say $O$, has $<d$ elements. Choose a subset $Y$ of $X$ with $d$ elements so that $Y$ contains $O$. For all $g \in \mathfrak{G}$, $O \subset g Y$. Namely, $O$ is contained in every subset of $X$ of cardinality $d$. The complement of $O$ in $X$ has $>(2 d-1)-d=d-1$ elements. Hence $X \backslash O$ contains at least one set of cardinality $d$. A contradiction.

Fix a point $o \in X$. Then

- The stabilizer $\mathfrak{G}_{\text {o }}$ of o in $\mathfrak{G}$ is a maximal subgroup of $\mathfrak{G}$ :

Assume $\mathfrak{G}_{o}<\mathfrak{H}<\mathfrak{G}$ for a group $\mathfrak{H}$. Then $1<(\mathfrak{G}: \mathfrak{H}),\left(\mathfrak{H}: \mathfrak{G}_{o}\right)<d$, because

$$
(\mathfrak{G}: \mathfrak{H})\left(\mathfrak{H}: \mathfrak{G}_{o}\right)=\left(\mathfrak{G}: \mathfrak{G}_{o}\right)=\operatorname{Card}(\mathfrak{G} . o)=\operatorname{Card}(X)=2 d-1 .
$$

As $\mathfrak{H} . o \simeq \mathfrak{H} / \mathfrak{G}_{o}, X \backslash(\mathfrak{H} . o)$ has cardinality $>(2 d-1)-d=d-1$. Pick a set $Y \subset X \backslash(\mathfrak{H} . o)$ with $d$ elements. Then $g Y \cap g \mathfrak{H} . o=\emptyset, \forall g \in \mathfrak{G}$. Therefore, each subset of $X$ of cardinality $d$ is disjoint with at least one translate $g \mathfrak{H} . o$ of $\mathfrak{H}$.o. Let $\mathfrak{R}$ be a set of representatives for $\mathfrak{G} / \mathfrak{H}$, which has cardinality $(\mathfrak{G}: \mathfrak{H})<d$. So $\mathfrak{R} . o$ is contained in some set of cardinality $d$ in $X$, say $Z$. But $Z$ intersects every $g \mathfrak{H} . o, \forall g \in \mathfrak{G}$. A contradiction.

- The group $\mathfrak{G}_{o}$ contains no normal subgroups of $\mathfrak{G}$ other than 1 :

Let $\mathfrak{N}$ be a subgroup of $\mathfrak{G}_{o}$ such that $\mathfrak{N}$ is normal in $\mathfrak{G}$. Then $\mathfrak{N} g . o=g \mathfrak{N} . o=g . o, \forall g \in \mathfrak{G}$. That is, $\mathfrak{N}$ fixes every element of $\mathfrak{G} . o=X$. So $\mathfrak{N}=1$.

Let $\mathfrak{U}$ be the last term $>1$ in the derived series of $\mathfrak{G}$. Then $[\mathfrak{U}, \mathfrak{U}]=1$, as $\mathfrak{G}$ is solvable. So $\mathfrak{U}$ is abelian on which $\mathfrak{G}$ acts by conjugation. Let $V \subset \mathfrak{U}$ be a simple sub- $\mathfrak{G}$-module ; it is an $\mathbf{F}_{p}$-vector space for some prime number $p$. Let $f=\operatorname{dim} V$.

- One has $V \mathfrak{G}_{o}=\mathfrak{G}$ and $V \cap \mathfrak{G}_{o}=1$ :

The maximal subgroup $\mathfrak{G}_{o}$ does not contain $V$, as $V$ is normal in $\mathfrak{G}$. So $V \mathfrak{G}_{o}$ contains $\mathfrak{G}_{o}$ properly and so $V \mathfrak{G}_{o}=\mathfrak{G}$. The intersection $V \cap \mathfrak{G}_{o}$ is normalized by $\mathfrak{G}_{o}$ and by $V, V$ being abelian, and thus by 
$V \mathfrak{G}_{o}=\mathfrak{G}$. So $V \cap \mathfrak{G}_{o}$ is a sub- $\mathfrak{G}$-module of $V$ distinct from $V$. So $V \cap \mathfrak{G}_{o}=1$.

- The map $V \rightarrow X, v \mapsto v . o$, is a bijection :

It is surjective because $X=\mathfrak{G} . o=V \mathfrak{G}_{o} . o=V . o$. It is injective because if $v . o=v^{\prime} . o$, then $v^{-1} v^{\prime} \in V \cap \mathfrak{G}_{o}=1$ and $v=v^{\prime}$.

Now $p^{f}=\operatorname{Card}(V)=\operatorname{Card}(X)=2 d-1$. So $p>2$.

- The representation $\mathfrak{G}_{o} \rightarrow \mathrm{GL}(V), g \mapsto \operatorname{Int}(g)$, is faithful :

Let $g \in \mathfrak{G}_{o}$ be such that $\operatorname{Int}(g)=1$ on $V$. Then $g v \cdot o=g v g^{-1} . o=$ $\operatorname{Int}(g)(v) . o=v . o, \forall v \in V$. So $g$ fixes each point of $V . o=X$.

Pick a prime $p^{\prime}$ such that $d<p^{\prime}<2 d$ (Bertrand's postulate).

- Then $p^{\prime}=p$ :

Suppose $p^{\prime} \neq p$. By its choice, $p^{\prime}$ divides $N:=\left(\begin{array}{c}2 d-1 \\ d\end{array}\right)$. Notice that $X$ has $N$ subsets of cardinality $d$. These $N$ subsets are permuted transitively by $\mathfrak{G}$. And $\mathfrak{G}=\mathfrak{G}_{o} V$ imbeds into $\mathrm{GL}(V) V$ by the faithful representation Int : $\mathfrak{G}_{o} \hookrightarrow \operatorname{GL}(V)$. So $p^{\prime}$ divides the order of $\mathrm{GL}(V) V$. So $p^{\prime}$ divides $p^{i}-1$ for some $i \in\{1, \cdots, f\}$, as $p^{\prime} \neq p$. But this is absurd. For, $p^{\prime}$ is odd, $p^{i}-1$ is even and $p^{i}-1 \leq p^{f}-1=2 d-2<2 p^{\prime}-2$.

- Then $f=1$ :

For, $p^{f}=2 d-1<2 p^{\prime}-1=2 p-1$.

- One has $d \leq 3$ :

This is immediate from the division :

$$
\begin{aligned}
& \left(\begin{array}{c}
2 d-1 \\
d
\end{array}\right) \mid \operatorname{Card}(\mathrm{GL}(V) V)=p(p-1)=(2 d-1)(2 d-2) . \\
& \text { — Case } d=2 \text {. Then } \mathfrak{G}=\mathfrak{S}_{3} \text { or } \mathfrak{A}_{3} \text { on } X=\{1,2,3\}:
\end{aligned}
$$

The set $X$ has $2 d-1=3$ elements. The transitivity of the $\mathfrak{G}$ action on the 2-point subsets of $X$ is equivalent to the transitivity of the $\mathfrak{G}$-action on $X$. So $\mathfrak{G}$ is either $\mathfrak{S}_{3}$ or $\mathfrak{A}_{3}$.

- Case $d=3$. Then $\mathfrak{G}$ consists of all affine linear transformations of $\mathbf{F}_{5}=X$ :

The set $X$ as well as $V$ has $2 d-1=5$ elements. So $V=\mathbf{F}_{5}$. And $\mathrm{GL}(V) V$ is the group of all affine linear transformations of $\mathbf{F}_{5}$ which acts 2 -transitively on $\mathbf{F}_{5}$. Indeed, if $a, b$ are two distinct points of $\mathbf{F}_{5}$, the affine linear transformation $x \mapsto(a-b) x+b$ maps 0 to $b$ and maps 1 to $a$. In particular, $\mathrm{GL}(V) V$ permutes transitively the 2-point subsets, or what amounts to the same, the 3 -point subsets, of $\mathbf{F}_{5}$. 
The unique index 2 subgroup $H$ of $\mathrm{GL}(V) V$ consists of all transformations of the form :

$$
x \mapsto a^{2} x+b, \forall x \in \mathbf{F}_{5}
$$

where $a \in \mathbf{F}_{5}^{\times}, b \in \mathbf{F}_{5}$. The 2-point subsets $\{u, v\}$ of $\mathbf{F}_{5}$ are divided into $2 H$-orbits according to whether or not $u-v$ is a square in $\mathbf{F}_{5}^{\times}$. Notice that -1 is a square in $\mathbf{F}_{5}^{\times}$. The assertion evidently follows.

Lemma 6.4. With the notations and assumptions of (6.1), let $o \in X$ be a point and $\mathfrak{G}_{o}$ its stabilizer in $\mathfrak{G}$. Suppose furthermore that $\mathfrak{G}$ acts transitively on $X$ and that the following condition holds :

- There is a subgroup $\mathfrak{H}$ of even index in $\mathfrak{G}$ such that $\mathfrak{H}$ contains $\mathfrak{G}_{\text {o }}$ properly.

Then the following list enumerates such $(X, \mathfrak{G})$ up to equivalence:

1) $X=\mathbf{Z} / 4 \mathbf{Z}, \mathfrak{G}$ consists of either all transformations

$$
x \mapsto a x+b, \forall x \in \mathbf{Z} / 4 \mathbf{Z}
$$

where $a \in(\mathbf{Z} / 4 \mathbf{Z})^{\times}, b \in \mathbf{Z} / 4 \mathbf{Z}$ or only of the translations

$$
x \mapsto x+b, \forall x \in \mathbf{Z} / 4 \mathbf{Z}
$$

where $b \in \mathbf{Z} / 4 \mathbf{Z}$.

2) $X=\{1, \cdots, 6\}, \mathfrak{G}$ is either the normalizer $\mathfrak{N}$ in $\operatorname{Aut}(X)$ of a partition $X=\{a, b, c\} \cup\left\{a^{\prime}, b^{\prime}, c^{\prime}\right\}$ or it is the subgroup of $\mathfrak{N}$ generated by $\mathfrak{A} \mathfrak{t}(\{a, b, c\}) \mathfrak{A} \mathfrak{t}\left(\left\{a^{\prime}, b^{\prime}, c^{\prime}\right\}\right)$ and one of the following subgroups :

$-\left\langle\left(a a^{\prime}\right)\left(b b^{\prime}\right)\left(c c^{\prime}\right)\right\rangle$

$-\left\langle\left(a a^{\prime} b b^{\prime}\right)\left(c c^{\prime}\right)\right\rangle$

$-\left\langle\left(a a^{\prime}\right)\left(b b^{\prime}\right)\left(c c^{\prime}\right),(a b)\left(a^{\prime} b^{\prime}\right)\right\rangle$

Proof. Let $(\mathfrak{G}: \mathfrak{H})=2 r$ and let $\mathfrak{R}=\left\{g_{1}, \cdots, g_{2 r}\right\}$ be a set of representatives for $\mathfrak{G} / \mathfrak{H}$. Notice that

$$
d=\frac{\operatorname{Card}(X)}{2}=\frac{(\mathfrak{G}: \mathfrak{H})}{2}\left(\mathfrak{H}: \mathfrak{G}_{o}\right)=r \operatorname{Card}(\mathfrak{H} . o) \geq 2 r .
$$

In particular, if $\mathfrak{I}$ is a subset of $\mathfrak{R}$ of cardinality $r$, then

$$
Z=\mathfrak{I} \mathfrak{H} . o
$$

has $d$ elements.

As $\operatorname{Card}(\mathfrak{R} . o) \leq \operatorname{Card}(\mathfrak{R})=2 r \leq d$, there is some set $Z^{\prime}$ in $X$ with $d$ elements which contains $\mathfrak{R}$.o. By its choice $Z^{\prime}$ intersects every $g \mathfrak{H} . o$, $\forall g \in \mathfrak{G}$.

Since $\mathfrak{G}$ permutes the $d$-point subsets of $X$ in 2 orbits, each of these sets satisfies one or the other of the following conditions : 
i) It is equal to $\mathfrak{I} \mathfrak{H} . o$ for a subset $\mathfrak{I}$ of $\mathfrak{R}$, where $\mathfrak{I}$ has $r$ elements.

ii) It intersects every translate $g \mathfrak{H} . o, \forall g \in \mathfrak{G}$.

- Then $r=1$ :

Assume $r>1$. Then the set

$$
E:=\left\{g_{1}, \cdots, g_{r}\right\} \mathfrak{H} . o \cup\left\{g_{r+1} . o\right\} \backslash\left\{g_{1} . o\right\}
$$

has $d$ elements and is disjoint with $g_{2 r} \mathfrak{H} . o$. But $E$ is not of the form $\mathfrak{I} \mathfrak{H}$. o for any subset $\mathfrak{I}$ of $\mathfrak{R}$.

So $\mathfrak{R}=\left\{g_{1}, g_{2}\right\}, \operatorname{Card}(\mathfrak{H} . o)=d, X=\mathfrak{H} . o \cup \tau \mathfrak{H} . o$, where $\tau:=g_{1}^{-1} g_{2}$, and the $d$-point subsets of $X$ distinct from $\mathfrak{H} . o$ and $\tau \mathfrak{H} . o$ are permuted transitively by $\mathfrak{G}$.

- Then $d \leq 3$ :

Suppose $d>3$. Choose a point $o^{\prime} \in \mathfrak{H} . o \backslash\{o\}$. Both sets

$$
Y=\{o\} \cup \tau \mathfrak{H} . o \backslash\{\tau . o\}, Y^{\prime}=\left\{o, o^{\prime}\right\} \cup \tau \mathfrak{H} . o \backslash\left\{\tau . o, \tau . o^{\prime}\right\}
$$

have $d$ elements. Both are distinct from $\mathfrak{H} . o$ and $\tau \mathfrak{H}$.o. But $Y \neq g Y^{\prime}$, $\forall g \in \mathfrak{G}$. For, $Y \cap \mathfrak{H} . o$ consists of 1 element, while $g Y^{\prime} \cap \mathfrak{H} . o=$ $g\left(Y^{\prime} \cap g^{-1} \mathfrak{H} . o\right)$ consists of either 2 or $d-2$ elements, $\forall g \in \mathfrak{G}$.

- Case $d=2$ :

The set $X$ has $2 d=4$ elements. Both $\mathfrak{H} . o$ and $\tau \mathfrak{H} . o$ have 2 elements. As $\mathfrak{H}$ is a subgroup of $\operatorname{Aut}(\mathfrak{H} . o) \times \operatorname{Aut}(\tau \mathfrak{H} . o)$, it has 2 or 4 elements.

Suppose first that $\mathfrak{H}$ has 2 elements. Then $\mathfrak{G}_{o}=1,|\mathfrak{G}|=4$ and $\mathfrak{G}$ acts simply transitively on $X$.

Notice that the translation action on itself of $\mathbf{Z} / 4 \mathbf{Z}$ permutes the 2-point subsets $\{u, v\}$ of $\mathbf{Z} / 4 \mathbf{Z}$ in 2 orbits according to whether or not $u-v$ belongs to the subgroup $\mathfrak{H}=2 \mathbf{Z} / 4 \mathbf{Z}$. And, the translation action on itself of $\mathbf{Z} / 2 \mathbf{Z} \times \mathbf{Z} / 2 \mathbf{Z}$ permutes its 2 -point subsets $\{u, v\}$ in 3 orbits according to which subgroup $u-v$ generates.

Suppose next that $\mathfrak{H}$ has 4 elements. Then $\mathfrak{G}$ is a 2-Sylow subgroup of $\operatorname{Aut}(X)$. It is isomorphic to the group of all transformations :

$$
x \mapsto a x+b, \forall x \in \mathbf{Z} / 4 \mathbf{Z}
$$

where $a \in(\mathbf{Z} / 4 \mathbf{Z})^{\times}, b \in \mathbf{Z} / 4 \mathbf{Z}$. The group $\mathfrak{G}$ permutes the 2-point subsets $\{u, v\}$ of $\mathbf{Z} / 4 \mathbf{Z}$ in 2 orbits according to whether or not $u-v$ lies in $2 \mathbf{Z} / 4 \mathbf{Z}$.

- Case $d=3$ : 
The set $X$ has $2 d=6$ elements. Both $\mathfrak{H} . o$ and $\tau \mathfrak{H} . o$ have 3 elements. Let $\mathfrak{N}$ denote the normalizer in $\operatorname{Aut}(X)$ of the partition

$$
X=\mathfrak{H} . o \cup \tau \mathfrak{H} . o .
$$

The group $\mathfrak{N}$ has 72 elements. Besides $\mathfrak{H} . o$ and $\tau \mathfrak{H} . o$, there are 18 subsets in $X$ of cardinality 3 . These 18 sets are permuted transitively by $\mathfrak{G}$. So $\mathfrak{G}$ is of index 1,2 or 4 in $\mathfrak{N}$.

We write $\mathfrak{H} . o=\{1,2,3\}$ and $\tau \mathfrak{H} . o=\{4,5,6\}$.

Let $\mathfrak{P}:=\mathfrak{A} \mathfrak{t}(\{1,2,3\}) \times \mathfrak{A} \mathfrak{t}(\{4,5,6\})$. It is the unique 3-Sylow subgroup of $\mathfrak{N}$ and of $\mathfrak{G}$. Let $\mathfrak{Q}$ be a 2-Sylow subgroup of $\mathfrak{G}$. Thus $\mathfrak{G}=\mathfrak{P Q}$ and $\mathfrak{Q}$ is of order 2,4 or 8 .

i) Case $\operatorname{Card}(\mathfrak{Q})=2$ :

Let $\mathfrak{Q}=\{1, \alpha\}$, where $\alpha$ transforms $\{1,2,3\}$ to $\{4,5,6\}$. If say

$$
\alpha: 1 \mapsto 4,2 \mapsto 5,3 \mapsto 6
$$

then $\alpha=(14)(25)(36)$.

ii) Case $\operatorname{Card}(\mathfrak{Q})=4, \mathfrak{Q}$ cyclic:

Let $\alpha$ be a generator of $\mathfrak{Q}$ which then transforms $\{1,2,3\}$ to $\{4,5,6\}$. So $\alpha^{2}$ normalizes $\{1,2,3\}$ and, being of order $2, \alpha^{2}$ fixes a point, say 3 , in $\{1,2,3\}$. If say

$$
\alpha: 1 \mapsto 4,2 \mapsto 5,3 \mapsto 6
$$

then $\alpha=(1425)(36)$.

iii) Case $\operatorname{Card}(\mathfrak{Q})=4, \mathfrak{Q}$ non cyclic :

Let $\mathfrak{Q}=\{1, \alpha, \beta, \gamma\}$. Suppose that $\alpha$ and $\beta$ (resp. $\gamma$ ) transform $\{1,2,3\}$ to $\{4,5,6\}$ (resp. normalizes $\{1,2,3\}$ ). Then $\gamma$ fixes a point, say 3 , in $\{1,2,3\}$. If say

$$
\alpha: 1 \mapsto 4,2 \mapsto 5,3 \mapsto 6
$$

then $\alpha=(14)(25)(36), \gamma=(12)(45), \beta=(15)(24)(36)$.

iv) Case $\operatorname{Card}(\mathfrak{Q})=8$ :

Then $\mathfrak{G}=\mathfrak{N}$.

It remains to verify that in all these cases $\mathfrak{G}$ permutes transitively the 3-point subsets $Y$ of $X$ other than $\{1,2,3\}$ and $\{4,5,6\}$.

Given such a subset $Y$ of $X$, notice that there is an element $p \in \mathfrak{P}$ such that $p Y$ is either $\{1,2,6\}$ or $\{3,4,5\}$. Then, in the notations of i)-iii), $\alpha$ transforms $\{1,2,6\}$ to $\{3,4,5\}$. 
Lemma 6.5. With the notations and assumptions of (6.1), let $o \in X$ be a point and $\mathfrak{G}_{o}$ its stabilizer in $\mathfrak{G}$. Suppose furthermore that $\mathfrak{G}_{o}$ acts transitively on $X$ and that the following condition holds :

- There is a subgroup $\mathfrak{H}$ of odd index $>1$ in $\mathfrak{G}$ such that $\mathfrak{H}$ contains $\mathfrak{G}_{\text {o }}$ properly.

Then $X=\{1, \cdots, 6\}, \mathfrak{G}$ is either the normalizer $\mathfrak{N}$ in $\operatorname{Aut}(X)$ of a partition $X=\left\{a, a^{\prime}\right\} \cup\left\{b, b^{\prime}\right\} \cup\left\{c, c^{\prime}\right\}$ or it is the subgroup of $\mathfrak{N}$ generated by $\left\{\left(a a^{\prime}\right),\left(b b^{\prime}\right),\left(c c^{\prime}\right),(a b c)\left(a^{\prime} b^{\prime} c^{\prime}\right)\right\}$.

Proof. Let $r$ be an integer $\geq 1$ such that $(\mathfrak{G}: \mathfrak{H})=2 r+1$. Let $\mathfrak{R}=\left\{g_{1}, \cdots, g_{2 r+1}\right\}$ be a set of representatives for $\mathfrak{G} / \mathfrak{H}$.

The identity

$$
d=\frac{\operatorname{Card}(X)}{2}=\frac{(\mathfrak{G}: \mathfrak{H})}{2}\left(\mathfrak{H}: \mathfrak{G}_{o}\right)=\left(r+\frac{1}{2}\right) \operatorname{Card}(\mathfrak{H} . o),
$$

implies in particular that $\mathfrak{H}$.o has even, say $2 f$, elements.

Choose a subset $B \subset g_{r+1} \mathfrak{H} . o \backslash\left\{g_{r+1} . o\right\}$ of cardinality $f$. Then

$$
Y=\left\{g_{1}, \cdots, g_{r}\right\} \mathfrak{H} . o \cup B
$$

has $d$ elements.

As $\operatorname{Card}(\mathfrak{R}) \leq d, \mathfrak{R} . o$ is contained in some set $Y^{\prime}$ with $d$ elements. This $Y^{\prime}$ intersects every $g \mathfrak{H} . o, \forall g \in \mathfrak{G}$.

By assumption $\mathfrak{G}$ has 2 orbits in the collection of $d$-point subsets of $X$. Each of these sets satisfies thus one or the other of the following two conditions :

- It is equal to $\mathfrak{I} \mathfrak{H} . o \cup B^{\prime}$ for some subset $\mathfrak{I}$ of $\mathfrak{R}$ of cardinality $r$ and some subset $B^{\prime}$ of $z \mathfrak{H}$.o of cardinality $f$, where $z$ is an element of $\mathfrak{R} \backslash \mathfrak{I}$.

— It intersects every $g \mathfrak{H} . o, \forall g \in \mathfrak{G}$.

Notice that every such set $\mathfrak{I} \mathfrak{H} . o \cup B^{\prime}$ intersects precisely $r+1$ members among

$$
g_{1} \mathfrak{H} . o, \cdots, g_{2 r+1} \mathfrak{H} . \text { o. }
$$

- Then $f=1$ :

Assume $f>1$. Then the set

$$
E:=\left\{g_{1}, \cdots, g_{r-1}\right\} \mathfrak{H} . o \cup\left(g_{r} \mathfrak{H} . o \backslash\left\{g_{r} . o\right\}\right) \cup\left(B \cup\left\{g_{r+1} . o\right\}\right)
$$

has $d$ elements and is disjoint with $g_{2 r+1} \mathfrak{H} . o$. But $E$ is not of the form $\mathfrak{I} \mathfrak{H} . o \cup B^{\prime}$ for any $\mathfrak{I} \subset \mathfrak{R}$ of cardinality $r$, any $z \in \mathfrak{R} \backslash \mathfrak{I}$ and any $B^{\prime} \subset z \mathfrak{H} . o$ of cardinality $f$.

It follows that $B$ consists of $f=1$ element and that $d=2 r+1$. 
- Then $r=1$ :

Suppose $r>1$. Then the set

$$
F:=\left\{g_{1}, \cdots, g_{r-1}\right\} \mathfrak{H} . o \cup\left(g_{r} \mathfrak{H} . o \backslash\left\{g_{r} . o\right\}\right) \cup\left\{g_{r+1} . o\right\} \cup\left\{g_{2 r+1} . o\right\}
$$

has $d$ elements and is disjoint with $g_{r+2} \mathfrak{H}$.o. But $F$ intersects $r+2$, rather than $r+1$, members among

$$
g_{1} \mathfrak{H} . o, \cdots, g_{2 r+1} \mathfrak{H} . \text { o. }
$$

Hence, $d=2 r+1=3, \mathfrak{R}=\left\{g_{1}, g_{2}, g_{3}\right\}$, the set $\mathfrak{H} . o$ has 2 elements and the set $X$ has 6 elements.

In $X$ there are 20 subsets of cardinality 3. Among these, 8 members intersect all three cosets $g \mathfrak{H}, \forall g \in\left\{g_{1}, g_{2}, g_{3}\right\}$. So $|\mathfrak{G}|$ is divisible by 8 and by $20-8=12$. That is, $|\mathfrak{G}|$ is a multiple of 24 .

So $\mathfrak{G}$ is either the normalizer $\mathfrak{N}$ in $\operatorname{Aut}(X)$ of the partition

$$
X=g_{1} \mathfrak{H} . o \cup g_{2} \mathfrak{H} . o \cup g_{3} \mathfrak{H} . o=\left\{a, a^{\prime}\right\} \cup\left\{b, b^{\prime}\right\} \cup\left\{c, c^{\prime}\right\}
$$

or it is the index 2 subgroup $\mathfrak{M}$ of $\mathfrak{N}$ generated by

$$
\left(a a^{\prime}\right),\left(b b^{\prime}\right),\left(c c^{\prime}\right),(a b c)\left(a^{\prime} b^{\prime} c^{\prime}\right) .
$$

It remains to verify that $\mathfrak{M}$ as well as $\mathfrak{N}$ has 2 orbits in the collection of 3-point subsets of $X$ :

Let $Z$ be a subset of $X$ of cardinality 3. Then

i) either $Z$ intersects all three : $\left\{a, a^{\prime}\right\},\left\{b, b^{\prime}\right\},\left\{c, c^{\prime}\right\}$

ii) or $Z$ is disjoint with exactly one among $\left\{a, a^{\prime}\right\},\left\{b, b^{\prime}\right\},\left\{c, c^{\prime}\right\}$.

In the first case, there is an element $g \in\left\langle\left(a a^{\prime}\right),\left(b b^{\prime}\right),\left(c c^{\prime}\right)\right\rangle$ such that $g Z=\{a, b, c\}$. In the latter, there is an element $g \in\left\langle(a b c)\left(a^{\prime} b^{\prime} c^{\prime}\right)\right\rangle$ such that $g Z$ is either $\left\{a, a^{\prime}, b\right\}$ or $\left\{a, a^{\prime}, b^{\prime}\right\}$. Then note that the cycle $\left(b b^{\prime}\right)$ transforms $\left\{a, a^{\prime}, b\right\}$ to $\left\{a, a^{\prime}, b^{\prime}\right\}$.

Lemma 6.6. With the notations and assumptions of (6.1), let $o \in X$ be a point and $\mathfrak{G}_{o}$ its stabilizer in $\mathfrak{G}$. Suppose furthermore that $\mathfrak{G}$ acts transitively on $X$ and that $\mathfrak{G}_{o}$ is a maximal subgroup of $\mathfrak{G}$.

Then $X=\mathbf{F}_{8}, \mathfrak{G}$ consists of either all affine semi-linear transformations

$$
x \mapsto a x^{2^{c}}+b, \forall x \in \mathbf{F}_{8}
$$

where $a \in \mathbf{F}_{8}^{\times}, b \in \mathbf{F}_{8}, c \in \mathbf{Z} / 3 \mathbf{Z}$ or only of the affine linear transformations

$$
x \mapsto a x+b, \forall x \in \mathbf{F}_{8}
$$

where $a \in \mathbf{F}_{8}^{\times}, b \in \mathbf{F}_{8}$. 
Proof. As in (6.3) one argues that there exists a normal subgroup $V$ of $\mathfrak{G}$ which has the following properties:

$-\mathfrak{G}=V \mathfrak{G}_{o}, V \cap \mathfrak{G}_{o}=1$.

- $V$ acts simply transitively on $X$.

- $V$ is an $\mathbf{F}_{p}$-vector space for some prime $p$, and $\mathfrak{G}_{o}$ acts faithfully and irreducibly on $V$.

We identify $V$ with $X$ by the bijection $v \mapsto v . o$.

Let $f=\operatorname{dim} V$. Then $p^{f}=\operatorname{Card}(V)=\operatorname{Card}(X)=2 d$. So $p=2$ and $d=2^{f-1}$. Clearly, $f>1$.

- Then $f>2$ :

Suppose $f=2$. As $\mathfrak{G}_{o}$ acts irreducibly on $V$, it cannot be a 2group. So $\left|\mathfrak{G}_{o}\right|$ is divisible by 3 . So $\mathfrak{G}$ is $\operatorname{Aut}(X)=\mathfrak{S}_{4}$ or $\mathfrak{A}_{4}$. But both permute transitively the 2 -point subsets of $X$ rather than have 2 orbits.

One has now $d=2^{f-1} \geq 4$.

Notice that every hyperplane of $V$ has $2^{f-1}=d$ elements. If $H_{1}, H_{2}$ are two distinct hyperplanes, the intersection $H_{1} \cap H_{2}$ has dimension $f-2$ and cardinality $2^{f-2}=d / 2$. And $H_{2} \backslash H_{1}$ has $d / 2$ elements. Given every $g \in \mathfrak{G}$, either $g H$ or $V \backslash g H$ is a hyperplane. Hence $g H \backslash H$ has $0, d$ or $d / 2$ elements.

Fix a point $v \in V \backslash H$. The set

$$
Y=\{v\} \cup H \backslash\{0\}
$$

has $d$ elements. As $Y \backslash H$ consists only of one point, neither $Y$ nor its complement is a hyperplane.

Therefore, $\mathfrak{G} H$ and $\mathfrak{G} Y$ are these 2 orbits of $\mathfrak{G}$ in the collection of $d$-point subsets of $X$.

- Then $f=3$ :

Assume $f>3$. Choose a point $u \in H \backslash\{0\}$. The set

$$
Z=\{v, u+v\} \cup H \backslash\{0, u\}
$$

has $d$ elements. But $Z$ is not a member of $\mathfrak{G} H$ or $\mathfrak{G} Y$. For, if $g$ is an element of $\mathfrak{G}$, then

— the set $g H \backslash H$ has $0, d$ or $d / 2$ elements,

— the set $g Y \backslash H=g\left(Y \backslash g^{-1} H\right)$ has $1, d-1, d / 2,(d / 2)+1$ or $(d / 2)-1$ elements,

- while the set $Z \backslash H$ has 2 elements.

Note that $2 \notin\{0,1, d, d-1, d / 2,(d / 2)+1,(d / 2)-1\}$, as $d \geq 8$. 
So $f=3$, and $\mathbf{P}(V)=\mathbf{P}^{2}$ is a projective plane over $\mathbf{F}_{2}$ which has 7 $\mathbf{F}_{2}$-rational points. That is, $V$ has 7 hyperplanes. These hyperplanes are permuted transitively by $\mathfrak{G}$. So 7 divides $|\mathfrak{G}|$ and $\left|\mathfrak{G}_{o}\right|$.

If one identifies $V$ with the underlying group of a finite field $\mathbf{F}_{8}$, a 7-Sylow subgroup of $\mathfrak{G}_{o}$ consists of all scalar multiplications

$$
l_{a}: x \mapsto a x, \forall x \in \mathbf{F}_{8}
$$

where $a \in \mathbf{F}_{8}^{\times}$.

- The normalizer $\mathfrak{N}$ of the group $\left\{l_{a}, a \in \mathbf{F}_{8}^{\times}\right\}$in $\operatorname{GL}(V)$ consists of all transformations of the form :

$$
x \mapsto a F^{c}(x), \forall x \in \mathbf{F}_{8}^{\times}
$$

where $a \in \mathbf{F}_{8}^{\times}, c \in \mathbf{Z} / 3 \mathbf{Z}$ and $F: x \mapsto x^{2}, \forall x \in \mathbf{F}_{8}$, is the Frobenius.

Suppose that an element $g \in \operatorname{GL}(V)$ normalizes $\left\{l_{a}\right\}$. The characteristic polynomial of $g l_{a} g^{-1}$ on $V$ factors as :

$$
\operatorname{det}\left(T-g l_{a} g^{-1}, V\right)=\operatorname{det}\left(T-l_{a}, V\right)=(T-a)\left(T-a^{2}\right)\left(T-a^{4}\right) .
$$

There is thus an element $c \in \mathbf{Z} / 3 \mathbf{Z}$ such that

$$
g l_{a} g^{-1}=l_{F^{c}(a)}=F^{c} l_{a} F^{-c} .
$$

So $F^{-c} g$ commutes with all elements of the cyclic group $\left\{l_{a}\right\}$. So $F^{-c} g$ belongs to $\left\{l_{a}\right\}$. That is to say, $g$ is of the form

$$
x \mapsto a F^{c}(x), \forall x \in \mathbf{F}_{8}
$$

for some $a \in \mathbf{F}_{8}^{\times}$and $c \in \mathbf{Z} / 3 \mathbf{Z}$. In particular, $\mathfrak{N}$ has 21 elements.

- The group $\mathfrak{G}_{o}$ is of odd order :

As $\mathfrak{G}_{o}$ is solvable, it has a Hall subgroup $\mathfrak{H}$ which is generated by $\left\{l_{a}\right\}$ and a 2-Sylow subgroup $\mathfrak{Q}$ of $\mathfrak{G}_{o}$. Assume that $\mathfrak{H}$ is not of odd order. Thus $\mathfrak{H}$ is not a subgroup of $\mathfrak{N}$. That is, $\left\{l_{a}\right\}$ is not normal in $\mathfrak{H}$. So $\mathfrak{Q}$ is not of order 2 or 4 . As $\operatorname{GL}(V)$ is of order $2^{3} .3 .7, \mathfrak{Q}$ is of order 8 and thus is normal in $\mathfrak{H}$. As $\mathfrak{Q}$ is 2 -Sylow in $\operatorname{GL}(V)$, the center $\mathfrak{Z}$ of $\mathfrak{Q}$ is of order 2 , which is normalized by $\left\{l_{a}\right\}$ and thus is centralized by $\left\{l_{a}\right\}$ and thus is contained in $\mathfrak{N}$. This is absurd.

Now $\left|\mathfrak{G}_{o}\right|=7$ or 21. In particular, $\left\{l_{a}\right\}$ is normal in $\mathfrak{G}_{o}$. Hence, $\mathfrak{G}_{o}$ is contained in $\mathfrak{N}$. So $\mathfrak{G}_{o}$ is either $\mathfrak{N}$ or $\left\{l_{a}\right\}$. So $\mathfrak{G}$ is either $V \mathfrak{N}$, the group of all affine semi-linear transformations

$$
x \mapsto a x^{2^{c}}+b, \forall x \in \mathbf{F}_{8}
$$

where $a \in \mathbf{F}_{8}^{\times}, b \in \mathbf{F}_{8}, c \in \mathbf{Z} / 3 \mathbf{Z}$ or it is $V\left\{l_{a}\right\}$ which consists of all affine linear transformations

$$
x \mapsto a x+b, \forall x \in \mathbf{F}_{8}
$$


where $a \in \mathbf{F}_{8}^{\times}, b \in \mathbf{F}_{8}$.

It remains to verify that $V\left\{l_{a}\right\}$ as well as $V \mathfrak{N}$ has 2 orbits in the collection of 4-point subsets of $\mathbf{F}_{8}$ :

There are 70 these subsets. Among them, the 7 hyperplanes and their complements, 14 in number, form 1 orbit under $V\left\{l_{a}\right\}$. For, if $E$ is one such set, then by a translation if necessary, one can transform $E$ to a hyperplane $H$. Now all 7 hyperplanes are of the form $a H$, for $a \in \mathbf{F}_{8}^{\times}$.

Let $Y$ be a member in the rest $70-14=56$ sets of cardinality 4 . Let $\mathfrak{S}$ denotes the normalizer of $Y$ in $V\left\{l_{a}\right\}$. Thus $\mathfrak{S}$ is also a subgroup of $\operatorname{Aut}(Y) \times \operatorname{Aut}(X \backslash Y)=\mathfrak{S}_{4} \times \mathfrak{S}_{4}$, whose order is not a multiple of 7. So $\mathfrak{S}$ consists only of translations. So $\mathfrak{S}=1$ by the choice of $Y$. Therefore, these 56 subsets are permuted simply transitively by $V\left\{l_{a}\right\}$.

Lemma 6.7. Let $X$ a finite nonempty set of even cardinality $2 d$. Let $\mathfrak{G}$ be a solvable subgroup of $\operatorname{Aut}(X)$ which permutes transitively the subsets of $X$ of cardinality $d$.

Then up to equivalence $(X, \mathfrak{G})$ is one of the following :

1) $X=\{1,2\}, \mathfrak{G}=\mathfrak{S}_{2}$.

2) $X=\{1,2,3,4\}, \mathfrak{G}=\mathfrak{S}_{4}$ or $\mathfrak{A}_{4}$.

Proof. Let $o \in X$ be a point and $\mathfrak{G}_{o}$ its stabilizer in $\mathfrak{G}$. As in (6.3) there exists a normal subgroup $V$ of $\mathfrak{G}$ which has the following properties :

$-\mathfrak{G}=V \mathfrak{G}_{o}, V \cap \mathfrak{G}_{o}=1$.

- $V$ acts simply transitively on $X$.

- $V$ is an $\mathbf{F}_{p}$-vector space for some prime $p$, and $\mathfrak{G}_{o}$ acts faithfully and irreducibly on $V$.

We identify $V$ with $X$ by the bijection $v \mapsto v . o$. Let $f=\operatorname{dim} V$.

Then $p^{f}=\operatorname{Card}(V)=\operatorname{Card}(X)=2 d$. So $p=2$ and $d=2^{f-1}$.

- Then $f \leq 2$ :

Fix a hyperplane $H$ in $V$ and let $v$ be a vector in the complement of $H$. By assumption each subset of $V$ of cardinality $d$ is a transform of $H$ by an element of $\mathfrak{G}$. These subsets are thus hyperplanes or complements of hyperplanes. But if $f>2$, the set

$$
Y=\{v\} \cup H \backslash\{0\}
$$

is neither a hyperplane nor the complement of a hyperplane.

- Case $f=1$ : 
The set $X$ has $2^{f}=2$ elements. And $\mathfrak{G}$ permutes the subsets of $X$ of cardinality $d=1$ transitively. Hence, $\mathfrak{G}=\operatorname{Aut}(X)$.

- Case $f=2$ :

The set $X$ as well as $V$ has $2^{f}=4$ elements. On $V$ the group $\mathfrak{G}_{o}$ acts irreducibly. So $\mathfrak{G}_{o}$ cannot be a 2-group. So $\mathfrak{G}=V \mathfrak{G}_{o}$ is either $\operatorname{Aut}(X)$ or $\mathfrak{A} \mathfrak{t}(X)$. Both do permute transitively the 2-point subsets of $X$.

Proposition 6.8. Let $X$ a finite set of even cardinality $2 d \geq 4$. Let $\mathfrak{G}$ be a solvable subgroup of $\{1,-1\} \times \operatorname{Aut}(X)$ which permutes transitively the subsets of $X$ of cardinality $d$, where -1 transforms every subset $Y$ of $X$ of cardinalityd to $X \backslash Y$. Suppose furthermore that $\mathfrak{G}$ is not a subgroup of $\operatorname{Aut}(X)$.

The following list enumerates such $(X, \mathfrak{G})$ up to equivalence :

1) $X=\{1,2,3,4\}, \mathfrak{G}=\{1,-1\} \times \mathfrak{S}_{4}$.

2) $X=\{1,2,3,4\}, \mathfrak{G}=\{1,-1\} \times \mathfrak{A}_{4}$.

3) $X=\{1,2,3,4\}, \mathfrak{G}$ consists of $\mathfrak{A}_{4}$ and of all elements of the form $-1 . \alpha$, where $\alpha$ is an odd permutation of $X$.

4) $X=\{o, 1,2,3\}, \mathfrak{G}=\{1,-1\} \times \operatorname{Aut}(\{1,2,3\})$.

5) $X=\{o, 1,2,3\}, \mathfrak{G}=\{1,-1\} \times \mathfrak{A} \mathfrak{t}(\{1,2,3\})$.

6) $X=\{o, 1,2,3\}, \mathfrak{G}$ consists of $\mathfrak{A} \mathfrak{t}(\{1,2,3\})$ and of all elements of the form -1. $\alpha$ where $\alpha$ is an odd permutation of $\{1,2,3\}$.

7) $X=\{o\} \cup \mathbf{F}_{5}, \mathfrak{G}=\{1,-1\} \times \mathfrak{H}$ where $\mathfrak{H}$ is the group of affine linear transformations of $\mathbf{F}_{5}$.

Proof. Let $\mathfrak{H}=\mathfrak{G} \cap \operatorname{Aut}(X)$, which is of index 2 in $\mathfrak{G}$, as by assumption $\mathfrak{G}$ is not contained in $\operatorname{Aut}(X)$. It follows that

The collection of d-point subsets of $X$ are permuted by $\mathfrak{H}$ either transitively or in 2 orbits of the same cardinality.

- Case where $\mathfrak{H}$ permutes transitively :

By (6.7) the set $X$ has 4 elements and $\mathfrak{H}=\operatorname{Aut}(X)$ or $\mathfrak{A l t}(X)$.

i) If $\mathfrak{H}=\operatorname{Aut}(X)$, then $\mathfrak{G}=\{1,-1\} \times \operatorname{Aut}(X)$.

ii) If $\mathfrak{H}=\mathfrak{A} \mathfrak{l}(X)$, then either $\mathfrak{G}$ is $\{1,-1\} \times \mathfrak{A} \mathfrak{l}(X)$ or it consists of $\mathfrak{A} \mathfrak{t}(X)$ and of all elements of the form -1. $\alpha$, where $\alpha$ is an odd permutation of $X$.

- Case where $\mathfrak{H}$ permutes with 2 orbits of the same cardinality:

By the proof of (6.1) precisely the following two occur : 
- $X=\{o, 1,2,3\}, \mathfrak{H}$ fixes o and on $\{1,2,3\}$ it is $\mathfrak{S}_{3}$ or $\mathfrak{A}_{3}$.

$-X=\{o\} \cup \mathbf{F}_{5}, \mathfrak{H}$ fixes $o$ and on $\mathbf{F}_{5}$ it is the group of affine linear transformations.

Let $\mathfrak{N}$ denote the normalizer of $\mathfrak{H}$ in $\{1,-1\} \times \operatorname{Aut}(X)$.

Suppose first that $X=\{o, 1,2,3\}$.

Then $\mathfrak{N}=\{1,-1\} \times \operatorname{Aut}(\{1,2,3\})$ for both groups $\operatorname{Aut}(\{1,2,3\})$ and $\mathfrak{A} \mathfrak{t}(\{1,2,3\})$. For, in $\operatorname{Aut}(X)$, the subgroup $\operatorname{Aut}(\{1,2,3\})$ is maximal and not normal.

Suppose next that $X=\{o\} \cup \mathbf{F}_{5}$.

Then $\mathfrak{N}=\{1,-1\} \times \mathfrak{H}$. Indeed, if $g \in \mathfrak{N} \cap \operatorname{Aut}(X)$, then $\mathfrak{H} g . o=$ $g \mathfrak{H} . o=$ g.o. So $g . o=o$ and $g$ normalizes the subset $\mathbf{F}_{5}$. As $\mathfrak{H}$ acts 2 transitively on $\mathbf{F}_{5}$, there is an element $h \in \mathfrak{H}$ such that $h g$ fixes at least 2 points of $\mathbf{F}_{5}$. In particular, $h g$ is of order 1,2 or 3 . In $\mathfrak{H}$ the subgroup $\mathfrak{T}$ consisting of all translations is the unique 5-Sylow subgroup. So $\mathfrak{T}$ is normalized and thus is centralized by $h g$. It follows that $h g$ fixes all points of $\mathbf{F}_{5}$. So $h g=1$ and $g=h^{-1} \in \mathfrak{H}$.

The pair $(X, \mathfrak{G})$ appears hence in the following list :

iii) $X=\{o, 1,2,3\}, \mathfrak{G}=\{1,-1\} \times \operatorname{Aut}(\{1,2,3\})$.

iv) $X=\{o, 1,2,3\}, \mathfrak{G}=\{1,-1\} \times \mathfrak{A} \mathfrak{t}(\{1,2,3\})$.

v) $X=\{o, 1,2,3\}, \mathfrak{G}$ consists of $\mathfrak{A} \mathfrak{t}(\{1,2,3\})$ and of all elements of the form $-1 . \alpha$, where $\alpha$ is an odd permutation of $\{1,2,3\}$.

vi) $X=\{o\} \cup \mathbf{F}_{5}, \mathfrak{G}=\{1,-1\} \times \mathfrak{H}$ where $\mathfrak{H}$ is the group of affine linear transformations of $\mathbf{F}_{5}$.

One inspects in each of the cases iii)-vi) that $\mathfrak{G}$ permutes the $d$-point subsets of $X$ transitively.

Proposition 6.9. Let $(S, \eta, s)$ be as in $\S 4$. Then every $\left({ }^{2} A_{3}, \alpha_{2}\right)$ over $\eta$ is elliptic. If $n>5$, then $\left({ }^{2} A_{n}, \alpha_{\frac{n+1}{2}}\right)$ is not elliptic over $\eta$.

Proof. By $(3.1), 8)$ and $(6.8),\left({ }^{2} A_{n}, \alpha_{\frac{n+1}{2}}\right)$ is not elliptic over $\eta$ if $n>5$. Suppose $n=3$ and suppose given a $\left({ }^{2} A_{3}, \alpha_{2}\right)$ over $\eta$. Let

$$
\rho_{1}: \pi_{1}(\eta, \bar{\eta}) \rightarrow\{1,-1\}
$$

denote the index of ${ }^{2} A_{3}$. Let

$$
\rho_{2}: \pi_{1}(\eta, \bar{\eta}) \rightarrow \pi_{1}(S, \bar{\eta}) \rightarrow \mathfrak{A l l t}(\{1,2,3\})
$$

be a surjective homomorphism $(\S 4)$. Then

$$
\rho=\left(\rho_{1}, \rho_{2}\right): \pi_{1}(\eta, \bar{\eta}) \rightarrow\{1,-1\} \times \mathfrak{A} \mathfrak{H} \mathfrak{t}(\{1,2,3\})
$$


is surjective. Thus, by $(6.8), 5)+(3.1), 8)$, it follows that $\left({ }^{2} A_{3}, \alpha_{2}\right)$ is elliptic over $\eta$.

Proposition 6.10. Let $(S, \eta, s), \operatorname{char}(s)=\ell$, be as in $\S 4$. If $\ell=5$, then every $\left({ }^{2} A_{5}, \alpha_{3}\right)$ over $\eta$ is elliptic. When $(\ell, 5)=1, a\left({ }^{2} A_{5}, \alpha_{3}\right)$ over $\eta$ is elliptic if and only if ${ }^{2} A_{5}$ is ramified over $S$ and $\operatorname{Card}(k(s))$ $\bmod 5$ generates $\mathbf{F}_{5}^{\times}$.

Proof. Suppose given a $\left({ }^{2} A_{5}, \alpha_{3}\right)$ over $\eta$. Let

$$
\rho_{1}: \pi_{1}(\eta, \bar{\eta}) \rightarrow\{1,-1\}
$$

denote its index. By $(3.1), 8)+(6.8), 2)$ this $\left({ }^{2} A_{5}, \alpha_{3}\right)$ is elliptic if and only if there is a surjective homomorphism :

$$
\rho=\left(\rho_{1}, \rho_{2}\right): \pi_{1}(\eta, \bar{\eta}) \rightarrow\{1,-1\} \times \mathfrak{H}=: \mathfrak{G}
$$

where $\mathfrak{H}$ consists of all affine linear transformations of $\mathbf{F}_{5}$.

Notice that $\mathfrak{H}$ is a quotient of $\pi_{1}(\eta, \bar{\eta})$ if and only if one of the following two holds :

$-\ell=5(4.1)$.

$-(\ell, 5)=1$ and $\operatorname{Card}(k(s)) \bmod 5$ generates $\left.\mathbf{F}_{5}^{\times}(4.2), 2\right)$.

Note also that

If $(\ell, 5)=1$ and if $\rho_{1}$ is unramified over $S$, then $\left({ }^{2} A_{5}, \alpha_{3}\right)$ is not elliptic over $\eta$.

Otherwise, the image of the inertia subgroup of $\pi_{1}(\eta, \bar{\eta})$ in $\mathfrak{G}$ would be the subgroup $\mathfrak{T}$ of all translations of $\mathbf{F}_{5}$. But $\mathfrak{G} / \mathfrak{T}$ is not cyclic.

- Case $\ell=5, \rho_{1}$ unramified over $S$ :

Let $\pi \in \Gamma\left(S, \mathcal{O}_{S}\right)$ be a uniformizer. Then

$$
\eta[z, x] /\left(z^{4}-\pi, x^{5}-x-z^{-1}\right)
$$

is connected, totally ramified over $S$ and Galois over $\eta$ with Galois group $\mathfrak{H}$. If its corresponding monodromy representation is

$$
\rho_{2}: \pi_{1}(\eta, \bar{\eta}) \rightarrow \mathfrak{H}
$$

then

$$
\rho=\left(\rho_{1}, \rho_{2}\right): \pi_{1}(\eta, \bar{\eta}) \rightarrow \mathfrak{G}
$$

is surjective.

- Case $\ell=5, \rho_{1}$ ramified over $S$ :

Let $\pi \in \Gamma\left(S, \mathcal{O}_{S}\right)$ be a uniformizer. Let $S^{\prime}$ be the spectra of a discrete valuation ring such that $S^{\prime}$ is finite étale Galois over $S$ with cyclic Galois group of order $4, \eta^{\prime}$ (resp. $s^{\prime}$ ) the generic (resp. closed) 
point of $S^{\prime}, \zeta \in \operatorname{Gal}\left(S^{\prime} / S\right)$ a generator and let $u^{\prime} \in \Gamma\left(S^{\prime}, \mathcal{O}_{S^{\prime}}\right)^{\times}$a unit such that the images of $u^{\prime}, \cdots, \zeta^{3}\left(u^{\prime}\right)$ in $k\left(s^{\prime}\right)$ form a normal base over $k(s)$. Then

$$
\eta^{\prime}\left[x_{1}, \cdots, x_{4}\right] /\left(x_{1}^{5}-x_{1}-\zeta\left(u^{\prime}\right) \pi^{-1}, \cdots, x_{4}^{5}-x_{4}-\zeta^{4}\left(u^{\prime}\right) \pi^{-1}\right)
$$

is connected and Galois over $\eta$ with Galois group $\mathfrak{H}$. If its corresponding monodromy representation is

$$
\rho_{2}: \pi_{1}(\eta, \bar{\eta}) \rightarrow \mathfrak{H}
$$

then

$$
\rho=\left(\rho_{1}, \rho_{2}\right): \pi_{1}(\eta, \bar{\eta}) \rightarrow \mathfrak{G}
$$

is surjective.

- Case $(\ell, 5)=1, \operatorname{Card}(k(s)) \bmod 5$ generates $\mathbf{F}_{5}^{\times}, \rho_{1}$ ramified over $S:$

By $(4.2), 2) \mathfrak{H}$ is realizable as a tame quotient of $\pi_{1}(\eta, \bar{\eta})$, say

$$
\rho_{2}: \pi_{1}(\eta, \bar{\eta}) \rightarrow \mathfrak{H}
$$

Now

$$
\rho=\left(\rho_{1}, \rho_{2}\right): \pi_{1}(\eta, \bar{\eta}) \rightarrow \mathfrak{G}
$$

is surjective.

\section{Type $B$}

Let $S, \eta, s, \operatorname{char}(s)=\ell$, be as in $\S 4$.

Let $n$ be an integer $\geq 3$. Let $e_{1}, \cdots, e_{n}$ be the standard basis of $\mathbf{Z}^{n}$. We denote the group of diagonal (resp. monomial) matrices in $\mathrm{GL}_{n}(\mathbf{Z})$ by $\mathfrak{D}($ resp. $\mathfrak{M})$. Let $\mathfrak{W}=\mathfrak{D} \mathfrak{M}$.

Proposition 7.1. Suppose that $k(s)$ is of characteristic $\ell=2$. Then $\left(B_{n}, \alpha_{n}\right)$ is elliptic over $\eta$.

Proof. Let $\zeta \in \mathrm{GL}_{n}(\mathbf{Z})$ be such that

$$
\zeta: e_{1} \mapsto e_{2}, e_{2} \mapsto e_{3}, \cdots, e_{n} \mapsto e_{1}
$$

Let $\mathfrak{G}$ be the subgroup of $\mathfrak{W}$ generated by $\zeta$ and all diagonal matrices. The group $\mathfrak{G}$ permutes the vectors

$$
\pm e_{1} \pm \cdots \pm e_{n}
$$

transitively. Moreover, $\mathfrak{G}$ is a quotient of $\pi_{1}(\eta, \bar{\eta})(4.1)$. So $\left(B_{n}, \alpha_{n}\right)$ is elliptic over $\eta(3.1), 2)$. 
Proposition 7.2. The pair $\left(B_{3}, \alpha_{3}\right)$ is elliptic over $\eta$.

Proof. The following two elements of $\mathrm{GL}_{3}(\mathbf{Z})$

$$
\begin{aligned}
& a: e_{1} \mapsto e_{1}, e_{2} \mapsto e_{3}, e_{3} \mapsto-e_{2} \\
& b: e_{1} \mapsto-e_{1}, e_{2} \mapsto e_{2}, e_{3} \mapsto e_{3}
\end{aligned}
$$

satisfy the relations

$$
a^{4}=b^{2}=1, a b=b a .
$$

The group $\mathfrak{G}$ they generate is isomorphic to $\mathbf{Z} / 4 \mathbf{Z} \times \mathbf{Z} / 2 \mathbf{Z}$. One verifies that $\mathfrak{G}$ permutes the vectors

$$
\pm e_{1} \pm e_{2} \pm e_{3}
$$

simply transitively. Moreover, $\mathfrak{G}$ is a quotient of $\pi_{1}(\eta, \bar{\eta})$. Indeed, let

$$
\rho_{1}: \pi_{1}(\eta, \bar{\eta}) \rightarrow \pi_{1}(S, \bar{\eta}) \rightarrow \mathbf{Z} / 4 \mathbf{Z}
$$

be a surjective homomorphism $(\S 4)$ and let

$$
\rho_{2}: \pi_{1}(\eta, \bar{\eta}) \rightarrow \mathbf{Z} / 2 \mathbf{Z}
$$

be the monodromy representation corresponding to the quadratic extension

$$
k(\eta)[x] /\left(x^{2}-\pi\right)
$$

of $k(\eta)$, where $\pi \in \Gamma\left(S, \mathcal{O}_{S}\right)$ is a uniformizer. Then

$$
\rho=\left(\rho_{1}, \rho_{2}\right): \pi_{1}(\eta, \bar{\eta}) \rightarrow \mathbf{Z} / 4 \mathbf{Z} \times \mathbf{Z} / 2 \mathbf{Z}
$$

is surjective. By $(3.1), 2)\left(B_{3}, \alpha_{3}\right)$ is thus elliptic over $\eta$.

Proposition 7.3. The pair $\left(B_{4}, \alpha_{4}\right)$ is elliptic over $\eta$.

Proof. By (7.1) one can suppose $\ell>2$.

The following elements of $\mathrm{GL}_{4}(\mathbf{Z})$

$$
\begin{gathered}
a: e_{1} \mapsto e_{2}, e_{2} \mapsto-e_{1}, e_{3} \mapsto e_{3}, e_{4} \mapsto e_{4} \\
b: e_{1} \mapsto e_{1}, e_{2} \mapsto e_{2}, e_{3} \mapsto e_{4}, e_{4} \mapsto-e_{3} \\
c: e_{1} \mapsto e_{2}, e_{2} \mapsto e_{3}, e_{3} \mapsto e_{4}, e_{4} \mapsto-e_{1} \\
d: e_{1} \mapsto e_{3}, e_{2} \mapsto-e_{4}, e_{3} \mapsto-e_{1}, e_{4} \mapsto e_{2}
\end{gathered}
$$

satisfy the relations

$$
\begin{gathered}
a^{4}=b^{4}=1, a b=b a . \\
c^{8}=d^{4}=1, c d c^{-1}=d^{-1} .
\end{gathered}
$$

The group $\mathfrak{G}_{1}$ generated by $\{a, b\}$ is isomorphic to $\mathbf{Z} / 4 \mathbf{Z} \times \mathbf{Z} / 4 \mathbf{Z}$. The group $\mathfrak{G}_{2}$ generated by $\{c, d\}$ is quaternion of order 16 . Both permute simply transitively the vectors

$$
\pm e_{1} \pm e_{2} \pm e_{3} \pm e_{4}
$$


If $\operatorname{Card}(k(s)) \equiv 1 \bmod 4(\operatorname{resp} . \operatorname{Card}(k(s)) \equiv-1 \bmod 4)$, then $\mathfrak{G}_{1}$ (resp. $\left.\mathfrak{G}_{2}\right)$ is a quotient of $\pi_{1}^{t}(\eta, \bar{\eta})(\S 4)$. So $\left(B_{4}, \alpha_{4}\right)$ is elliptic over $\eta$ $(3.1), 2)$.

Proposition 7.4. Suppose $\ell>2, n>4$. Then $\left(B_{n}, \alpha_{n}\right)$ is not elliptic over $\eta$.

Proof. By $(3.1), 2)\left(B_{n}, \alpha_{n}\right)$ is elliptic if and only if there is a representation

$$
\rho: \pi_{1}(\eta, \bar{\eta}) \rightarrow \mathfrak{W}
$$

whose image permutes transitively the vectors

$$
\pm e_{1} \pm \cdots \pm e_{n} .
$$

Suppose that such a representation exists. Let $\mathfrak{G}$ be its image and $I$ (resp. $P$ ) the image of the inertia (resp. wild inertia) subgroup. Let

$$
X=\left\{ \pm e_{1} \pm \cdots \pm e_{n}\right\} .
$$

Observe that if an element $g$ of $\mathfrak{W}$ fixes all points of $X$, then $g=1$.

- One has $P=1$ :

For, $P$ being normal in $\mathfrak{G}$, the $P$-orbits in $X$ all have the same cardinality, say $r$, which divides both $2^{n}$ and $\operatorname{Card}(P)$. So $r=1$. So $P=1$.

Thus $I=I / P$ is cyclic.

- The cyclic group $I$ is a 2-group :

The maximal odd order subgroup of $I, I^{\prime}$, is normal in $\mathfrak{G}$. Thus the $I^{\prime}$-orbits in $X$ all have the same cardinality, say $r^{\prime}$, which divides both $2^{n}$ and $\operatorname{Card}\left(I^{\prime}\right)$. That is, $r^{\prime}=1$. So $I^{\prime}=1$.

Now, as $\mathfrak{G} / I$ is cyclic, $\mathfrak{G}$ has a unique 2-Sylow subgroup.

- The unique 2-Sylow subgroup $\mathfrak{H}$ of $\mathfrak{G}$ acts transitively on $X$ :

As $\mathfrak{H}$ is normal in $\mathfrak{G}$, the $\mathfrak{H}$-orbits in $X$ all have the same cardinality. These orbits, which form a set of cardinality dividing $2^{n}$, are permuted transitively by the quotient $\mathfrak{G} / \mathfrak{H}$. So there is only one orbit.

To $\mathfrak{H}$ there corresponds a subextension $k\left(\eta^{\prime}\right) / k(\eta)$ of $k(\bar{\eta}) / k(\eta)$ so that $\mathfrak{H}$ is the image of the composition

$$
\pi_{1}\left(\eta^{\prime}, \bar{\eta}\right) \rightarrow \pi_{1}(\eta, \bar{\eta}) \stackrel{\rho}{\longrightarrow} \mathfrak{G} .
$$

Replacing $\eta$ by $\eta^{\prime}$ if necessary, one can assume that $\mathfrak{G}=\mathfrak{H}$ is a 2-group. 
Consider the exact sequence

$$
1 \rightarrow I \cap \mathfrak{D} \rightarrow \mathfrak{G} \cap \mathfrak{D} \rightarrow \mathfrak{G} / I .
$$

Notice that

- the group $I \cap \mathfrak{D}$ has $\leq 2$ elements. For, $I \cap \mathfrak{D}$ is both cyclic and an elementary 2-group.

— the image $\mathfrak{Q}$ of $\mathfrak{G} \cap \mathfrak{D}$ in $\mathfrak{G} / I$ has $\leq 2$ elements. For, being a subgroup of $\mathfrak{G} / I, \mathfrak{Q}$ is cyclic. And being a quotient of $\mathfrak{G} \cap \mathfrak{D}, \mathfrak{Q}$ is an elementary 2-group.

Therefore, $\mathfrak{G} \cap \mathfrak{D}$ is of order 1,2 or 4 .

The quotient $\mathfrak{G} /(\mathfrak{G} \cap \mathfrak{D})$ is isomorphic to a group of monomial matrices. Thus this 2-group is of order $2^{e}$ for an integer $e \leq \operatorname{ord}_{2}(n !)$. One has $\operatorname{ord}_{2}(n !) \leq n-1$, where the equality holds if and only if $n$ is a power of 2 .

As $\mathfrak{G}$ acts transitively on $X$, one of the following three holds :

1) $\mathfrak{G} \cap \mathfrak{D}$ has 4 elements, $\mathfrak{G} /(\mathfrak{G} \cap \mathfrak{D})$ has $2^{n-2}$ elements and is 2-Sylow in $\mathfrak{M}, n$ is not a power of 2 .

2) $\mathfrak{G} \cap \mathfrak{D}$ has 2 elements, $\mathfrak{G} /(\mathfrak{G} \cap \mathfrak{D})$ has $2^{n-1}$ elements and is 2-Sylow in $\mathfrak{M}, n$ is a power of 2 .

3) $\mathfrak{G} \cap \mathfrak{D}$ has 4 elements, $\mathfrak{G} /(\mathfrak{G} \cap \mathfrak{D})$ has $2^{n-2}$ or $2^{n-1}$ elements and is of index $\leq 2$ in a 2-Sylow subgroup of $\mathfrak{M}, n$ is a power of 2 .

Next, from the exact sequence

$$
1 \rightarrow I /(I \cap \mathfrak{D}) \rightarrow \mathfrak{G} /(\mathfrak{G} \cap \mathfrak{D}) \rightarrow \mathfrak{G} / I(\mathfrak{G} \cap \mathfrak{D}) \rightarrow 1
$$

one deduces that $\mathfrak{G} /(\mathfrak{G} \cap \mathfrak{D})$ does not have elementary 2-subgroups of 2 -rank $\geq 3$. So

$-n \leq 5$ in case 1$)$

$-n \leq 4$ in case 2 )

$-n \leq 4$ in case 3 )

It remains to consider the case $n=5$ :

By 1) then $|\mathfrak{G} \cap \mathfrak{D}|=4,|I \cap \mathfrak{D}|=2,|\mathfrak{G}|=32$.

The group $\mathfrak{G} /(\mathfrak{G} \cap \mathfrak{D})$ has 8 elements and is 2-Sylow in $\mathfrak{M}=\mathfrak{S}_{5}$ and is an extension of the cyclic group $\mathfrak{G} / I(\mathfrak{G} \cap \mathfrak{D})$ by the cyclic group $I /(I \cap \mathfrak{D})$. So $I /(I \cap \mathfrak{D})$ has 4 elements. So $I$ has 8 elements and $\mathfrak{G} / I$ has 4 elements.

Let $t$ be a generator of $I$. Choose an element $f$ of $\mathfrak{G}$ such that its image in $\mathfrak{G} / I$ is a generator. Then $f t f^{-1}=t^{q}$ for an odd integer $q$. So $f^{2}$ commutes with $t$, for $f^{2} t f^{-2}=t^{q^{2}}=t$, as $q^{2} \equiv 1 \bmod 8$. 
Observe that $\mathfrak{G}$ normalizes the set

$$
Y=\left\{e_{1}, \cdots, e_{5},-e_{1}, \cdots,-e_{5}\right\}
$$

in which one and only one $I$-orbit $O=-O$ has 8 elements. Let $O^{\prime}=$ $Y \backslash O$, which consists of two eigenvectors of $t$.

Now $f$ normalizes $O$ as well as $O^{\prime}$. On $O^{\prime}, f^{2}$ acts as the identity. On $O, f^{2}$ acts as $t^{r}$ for an even integer $r$ since it commutes with $t$. So $f^{2}=t^{r} \in I$. So $\mathfrak{G} / I$ has $\leq 2$ elements. A contradiction.

\section{Type $C$}

Let $(S, \eta, s)$ be as in $\S 4$.

Proposition 8.1. For every integer $n \geq 1,\left(C_{n}, \alpha_{1}\right)$ is elliptic over $\eta$.

Proof. Let $\zeta, \tau \in \mathrm{GL}_{n}(\mathbf{Z})$ be such that

$$
\begin{gathered}
\zeta: e_{1} \mapsto e_{2}, e_{2} \mapsto e_{3}, \cdots, e_{n} \mapsto e_{1} \\
\tau: e_{1} \mapsto-e_{1}, e_{i} \mapsto e_{i}, \forall i>1
\end{gathered}
$$

where $e_{1}, \cdots, e_{n}$ denote the standard basis of $\mathbf{Z}^{n}$.

The cyclic group $\langle\tau \zeta\rangle$ generated by $\tau \zeta$ permutes the vectors

$$
e_{1}, \cdots, e_{n},-e_{1}, \cdots,-e_{n}
$$

simply transitively. And, $\langle\tau \zeta\rangle$ is a quotient of $\pi_{1}(\eta, \bar{\eta})(\S 4)$. So $\left(C_{n}, \alpha_{1}\right)$ is elliptic over $\eta(3.1), 3)$.

\section{TYPE $D$}

Let $S, \eta, s, \operatorname{char}(s)=\ell$, be as in $\S 4$.

Let $n$ be an integer $\geq 4$. Let $e_{1}, \cdots, e_{n}$ be the standard basis of $\mathbf{Z}^{n}$. We denote the group of diagonal (resp. monomial) matrices in $\mathrm{GL}_{n}(\mathbf{Z})$ by $\mathfrak{D}$ (resp. $\mathfrak{M})$. Let $\mathfrak{D}_{1}$ be the subgroup of $\mathfrak{D}$ consisting of all diagonal matrices of determinant 1 .

Let $\mathfrak{W}=\mathfrak{D M}$ and $\mathfrak{W}_{1}=\mathfrak{D}_{1} \mathfrak{M}$.

Proposition 9.1. Suppose that $n$ is even. Then $\left(D_{n}, \alpha_{1}\right)$ is elliptic over $\eta$. 
Proof. As $n$ is even, the diagonal matrix $-1 \in \mathrm{GL}_{n}(\mathbf{Z})$ has determinant 1. Let $\zeta \in \mathrm{GL}_{n}(\mathbf{Z})$ be such that

$$
\zeta: e_{1} \mapsto e_{2}, \cdots, e_{n} \mapsto e_{1} .
$$

The subgroup $\mathfrak{G}$ of $\mathfrak{W}_{1}$ generated by $\{\zeta,-1\}$ permutes the vectors

$$
e_{1}, \cdots, e_{n},-e_{1}, \cdots,-e_{n}
$$

simply transitively. And $\mathfrak{G}$, which is isomorphic to $\mathbf{Z} / n \mathbf{Z} \times \mathbf{Z} / 2 \mathbf{Z}$, is a quotient of $\pi_{1}(\eta, \bar{\eta})$. Indeed, let

$$
\rho_{1}: \pi_{1}(\eta, \bar{\eta}) \rightarrow \pi_{1}(S, \bar{\eta}) \rightarrow \mathbf{Z} / n \mathbf{Z}
$$

be a surjective homomorphism $(\S 4)$ and let

$$
\rho_{2}: \pi_{1}(\eta, \bar{\eta}) \rightarrow \mathbf{Z} / 2 \mathbf{Z}
$$

be the monodromy representation corresponding to the quadratic extension

$$
k(\eta)[x] /\left(x^{2}-\pi\right)
$$

of $k(\eta)$, where $\pi \in \Gamma\left(S, \mathcal{O}_{S}\right)$ is a uniformizer. Then

$$
\rho=\left(\rho_{1}, \rho_{2}\right): \pi_{1}(\eta, \bar{\eta}) \rightarrow \mathbf{Z} / n \mathbf{Z} \times \mathbf{Z} / 2 \mathbf{Z}
$$

is surjective. So $\left(D_{n}, \alpha_{1}\right)$ is elliptic over $\left.\eta(3.1), 4\right)$.

Proposition 9.2. Suppose $\ell=2$. Then $\left(D_{n}, \alpha_{1}\right)$ is elliptic over $\eta$.

Proof. Let $\zeta \in \mathrm{GL}_{n}(\mathbf{Z})$ be such that

$$
\zeta: e_{1} \mapsto e_{2}, \cdots, e_{n} \mapsto e_{1} .
$$

The subgroup $\mathfrak{G}$ of $\mathfrak{W}_{1}$ generated by $\zeta$ and all diagonal matrices of determinant 1 permutes transitively the vectors

$$
e_{1}, \cdots, e_{n},-e_{1}, \cdots,-e_{n} .
$$

Moreover, $\mathfrak{G}$ is a quotient of $\pi_{1}(\eta, \bar{\eta})(4.1)$. So $\left(D_{n}, \alpha_{1}\right)$ is elliptic over $\eta(3.1), 4)$.

Lemma 9.3. Every odd order subgroup of $\mathfrak{W}_{1}$ is conjugate to a subgroup of $\mathfrak{M}$.

Proof. Let $\mathfrak{H}$ be an odd order subgroup of $\mathfrak{W}_{1}$. Consider the split exact sequence

$$
1 \rightarrow \mathfrak{D}_{1} \rightarrow \mathfrak{W}_{1} \rightarrow \mathfrak{M} \rightarrow 1
$$

and let $\mathfrak{H}^{\prime}$ be the image of $\mathfrak{H}$ in $\mathfrak{M}$. Then $\mathfrak{D}_{1} \mathfrak{H}=\mathfrak{D}_{1} \mathfrak{H}^{\prime}=$ : $\mathfrak{G}$. As $H^{1}\left(\mathfrak{H}^{\prime}, \mathfrak{D}_{1}\right)=0$, every two splittings of the exact sequence

$$
1 \rightarrow \mathfrak{D}_{1} \rightarrow \mathfrak{G} \rightarrow \mathfrak{H}^{\prime} \rightarrow 1
$$


especially the ones corresponding to $\mathfrak{H}$ and to $\mathfrak{H}^{\prime}$, are conjugate to each other by an element of $\mathfrak{G}$.

Proposition 9.4. Suppose $\ell>2$ and that $n$ is odd $\geq 3$. Then $\left(D_{n}, \alpha_{1}\right)$ is not elliptic over $\eta$.

Proof. This conclusion holds when $n=3$ by $(5.3)+(5.4)$, as $\left(D_{3}, \alpha_{1}\right)=$ $\left(A_{3}, \alpha_{2}\right)$.

Suppose $n \geq 5$.

By $(3.1), 4)\left(D_{n}, \alpha_{1}\right)$ is elliptic if and only if there is a representation

$$
\rho: \pi_{1}(\eta, \bar{\eta}) \rightarrow \mathfrak{W}_{1}
$$

whose image acts transitively on the set

$$
X=\left\{e_{1}, \cdots, e_{n},-e_{1}, \cdots,-e_{n}\right\} .
$$

Suppose that $n$ is the smallest odd integer $\geq 5$ for a representation $\rho$ as such exists. Let $\mathfrak{G}$ be its image and $I$ (resp. $P$ ) the image of the inertia (resp. wild inertia) subgroup of $\pi_{1}(\eta, \bar{\eta})$. By (9.3) one may assume $P$ to be a subgroup of monomial matrices. Thus, $P$ normalizes

$$
X_{+}=\left\{e_{1}, \cdots, e_{n}\right\} .
$$

Notice that, $P$ being normal in $\mathfrak{G}$, the $P$-orbits in $X$ all have the same cardinality, say $r$, which divides both $\operatorname{Card}(P)$ and $n$. Let $d=$ $n / r$. Let $E_{1}, \cdots, E_{d}$ be the $P$-orbits in $X_{+}$; the other $P$-orbits are $-E_{1}, \cdots,-E_{d}$. These $P$-orbits are permuted transitively by $\mathfrak{G}$.

Let $g=\delta p$ be an element of $\mathfrak{G}$, where $\delta \in \mathfrak{D}_{1}, p \in \mathfrak{M}$. Let $E$ be a $P_{-}$ orbit in $X_{+}$. Suppose that $g(E)=\chi E^{\prime}$, where $\chi \in\{1,-1\}, E^{\prime} \subset X_{+}$. Then $p(E)=\chi \delta\left(E^{\prime}\right)$. Namely, $p(E)=E^{\prime}, \delta \mid E^{\prime}=\chi$.

It follows that when $E_{1}, \cdots, E_{d}$ is considered as a base of a free $\mathbf{Z}$-module $\mathbf{Z}^{d}$ the permutation action of $\mathfrak{G}$ on

$$
\left\{E_{1}, \cdots, E_{d},-E_{1}, \cdots,-E_{d}\right\}
$$

induces a representation of $\mathfrak{G}$ in $\mathrm{GL}_{d}(\mathbf{Z})$ whose image lies in the group generated by the diagonal matrices of determinant 1 and monomial matrices.

Thus, in view of the choice of $n$, one has $d=n$. So $r=1, P=1$.

So $I=I / P$ is cyclic. The maximal odd order subgroup of $I$, which is normal in $\mathfrak{G}$, is 1 by the same argument as for $P$. That is, $I$ is a cyclic 2-group.

As $I$ is normal in $\mathfrak{G}$ and is commutative, the $I$-orbits in $X$ all have the same cardinality $|I|$, which divides $2 n$. So $|I|=1$ or 2 . So $\mathfrak{G}$ is 
commutative of order $2 n$. The unique index 2 subgroup of $\mathfrak{G}$ is again 1 by the same argument as for $P$. So $n=1$. A contradiction.

Proposition 9.5. The pairs $\left(D_{n}, \alpha_{n-1}\right)$ and $\left(D_{n}, \alpha_{n}\right)$ are elliptic over $\eta$ if $n=4$ or 5 .

Proof. By comparing $(3.1), 5)$ with $(3.1), 2)$, it is evident that $\left(D_{n}, \alpha_{n-1}\right)$ and $\left(D_{n}, \alpha_{n}\right)$ are elliptic if $\left(B_{n-1}, \alpha_{n-1}\right)$ is elliptic. One now applies $(7.2)+(7.3)$.

Proposition 9.6. Suppose $\ell=2$. Then $\left(D_{n}, \alpha_{n-1}\right)$ and $\left(D_{n}, \alpha_{n}\right)$ are elliptic over $\eta$.

Proof. Let $\zeta \in \mathrm{GL}_{n}(\mathbf{Z})$ be such that

$$
\zeta: e_{1} \mapsto e_{2}, \cdots, e_{n} \mapsto e_{1} .
$$

Let $\mathfrak{G}$ be the subgroup of $\mathfrak{W}_{1}$ generated by $\zeta$ and $\mathfrak{D}_{1}$. The group $\mathfrak{G}$ acts transitively on

$$
\left\{s_{1} e_{1}+\cdots+s_{n} e_{n}, s_{i} \in\{1,-1\}, s_{1} \cdots s_{n}=-1\right\}
$$

and on

$$
\left\{s_{1} e_{1}+\cdots+s_{n} e_{n}, s_{i} \in\{1,-1\}, s_{1} \cdots s_{n}=1\right\} .
$$

Moreover, $\mathfrak{G}$ is a quotient of $\pi_{1}(\eta, \bar{\eta})$. Indeed, let $S^{\prime}$ be the spectra of a discrete valuation ring such that $S^{\prime}$ is finite étale Galois over $S$ with cyclic Galois group of order $n(\S 4), \eta^{\prime}$ (resp. $s^{\prime}$ ) the generic (resp. closed) point of $S^{\prime}, \pi \in \Gamma\left(S, \mathcal{O}_{S}\right)$ a uniformizer and let $u^{\prime} \in \Gamma\left(S^{\prime}, \mathcal{O}_{S^{\prime}}\right)^{\times}$ be a unit such that the images of $u^{\prime}, \zeta\left(u^{\prime}\right), \cdots, \zeta^{n-1}\left(u^{\prime}\right)$ in $k\left(s^{\prime}\right)$ form a normal basis over $k(s)$. Put $b^{\prime}:=1+u^{\prime} \pi$. Then

$$
\eta^{\prime}\left[z_{1}, \cdots, z_{n}\right] /\left(z_{1}^{2}-\frac{\zeta\left(b^{\prime}\right)}{b^{\prime}}, \cdots, z_{n}^{2}-\frac{\zeta^{n}\left(b^{\prime}\right)}{\zeta^{n-1}\left(b^{\prime}\right)}, 1-z_{1} \cdots z_{n}\right)
$$

is connected and Galois over $\eta$ with Galois group $\mathfrak{G}$. So $\left(D_{n}, \alpha_{n-1}\right)$ and $\left(D_{n}, \alpha_{n}\right)$ are elliptic $\left.\left.(3.1), 4\right), 5\right)$.

Proposition 9.7. Suppose $\ell>2, n>5$. Then $\left(D_{n}, \alpha_{n-1}\right)$ and $\left(D_{n}, \alpha_{n}\right)$ are not elliptic over $\eta$.

Proof. It suffices to consider $\left(D_{n}, \alpha_{n}\right)$ only. The same argument applies for $\left(D_{n}, \alpha_{n-1}\right)$. By $\left.(3.1), 5\right)\left(D_{n}, \alpha_{n}\right)$ is elliptic if and only if there is a representation

$$
\rho: \pi_{1}(\eta, \bar{\eta}) \rightarrow \mathfrak{W}_{1}
$$


whose image acts transitively on the set

$$
X=\left\{s_{1} e_{1}+\cdots+s_{n} e_{n}, s_{i} \in\{1,-1\}, s_{1} \cdots s_{n}=1\right\} .
$$

Suppose that such a representation $\rho$ exists. Let $\mathfrak{G}$ be its image and $I$ (resp. $P$ ) the image in $\mathfrak{G}$ of the inertia (resp. wild inertia) subgroup of $\pi_{1}(\eta, \bar{\eta})$.

As in (7.4), extending if necessary $k(\eta)$ to a finite extension $k\left(\eta^{\prime}\right)$ which is unramified over $S$ and of odd degree over $k(\eta)$, one can assume that $\mathfrak{G}$ is a 2 -group. In particular, $P=1$ and $I$ is cyclic.

Consider the exact sequence

$$
1 \rightarrow I \cap \mathfrak{D}_{1} \rightarrow \mathfrak{G} \cap \mathfrak{D}_{1} \rightarrow \mathfrak{G} / I .
$$

As both $I \cap \mathfrak{D}_{1}$ and $\mathfrak{G} / I$ are cyclic, the elementary 2-group $\mathfrak{G} \cap \mathfrak{D}_{1}$ is of order 1,2 or 4 . The quotient $\mathfrak{G} /\left(\mathfrak{G} \cap \mathfrak{D}_{1}\right)$, which is isomorphic to a group of monomial matrices, is of order $2^{e}$ for an integer $e \leq \operatorname{ord}_{2}(n !)$. Notice that $\operatorname{ord}_{2}(n !) \leq n-1$, where the equality holds if and only if $n$ is a power of 2 .

As $\mathfrak{G}$ acts transitively on $X$, one of the following five holds :

1) $\left|\mathfrak{G} \cap \mathfrak{D}_{1}\right|=2$ or $4, \mathfrak{G} /\left(\mathfrak{G} \cap \mathfrak{D}_{1}\right)$ has $2^{n-2}$ elements and is 2-Sylow in $\mathfrak{M}, n$ is not a power of 2 .

2) $\left|\mathfrak{G} \cap \mathfrak{D}_{1}\right|=4, \mathfrak{G} /\left(\mathfrak{G} \cap \mathfrak{D}_{1}\right)$ has $2^{n-3}$ elements and is of index $\leq 2$ in a 2-Sylow subgroup of $\mathfrak{M}, n$ is not a power of 2 .

3) $\left|\mathfrak{G} \cap \mathfrak{D}_{1}\right|=1,2$ or $4, \mathfrak{G} /\left(\mathfrak{G} \cap \mathfrak{D}_{1}\right)$ has $2^{n-1}$ elements and is 2-Sylow in $\mathfrak{M}, n$ is a power of 2 .

4) $\left|\mathfrak{G} \cap \mathfrak{D}_{1}\right|=2$ or $4, \mathfrak{G} /\left(\mathfrak{G} \cap \mathfrak{D}_{1}\right)$ has $2^{n-2}$ elements and is of index $\leq 2$ in a 2-Sylow subgroup of $\mathfrak{M}, n$ is a power of 2 .

5) $\left|\mathfrak{G} \cap \mathfrak{D}_{1}\right|=4, \mathfrak{G} /\left(\mathfrak{G} \cap \mathfrak{D}_{1}\right)$ has $2^{n-3}$ elements and is of index 1,2 or 4 in a 2-Sylow subgroup of $\mathfrak{M}, n$ is a power of 2 .

Next, the exact sequence

$$
1 \rightarrow I /\left(I \cap \mathfrak{D}_{1}\right) \rightarrow \mathfrak{G} /\left(\mathfrak{G} \cap \mathfrak{D}_{1}\right) \rightarrow \mathfrak{G} / I\left(\mathfrak{G} \cap \mathfrak{D}_{1}\right) \rightarrow 1
$$

implies that $\mathfrak{G} /\left(\mathfrak{G} \cap \mathfrak{D}_{1}\right)$ does not contain elementary 2-groups of 2-rank $\geq 3$. So

$-n \leq 5$ in case 1 )

$-n \leq 6$ in case 2 )

$-n \leq 4$ in case 3 )

$-n \leq 4$ in case 4 )

$-n \leq 8$ in case 5 ) 
Now as $P=1, \mathfrak{G}$ is a quotient of $\pi_{1}^{t}(\eta, \bar{\eta})=\langle F, T\rangle$. Let $t \in I$ (resp. $f)$ be the image of $T$ (resp. $F$ ). Then $f t f^{-1}=t^{q}$ where $q=\operatorname{Card}(k(s))$ is a power of $\ell$.

Notice that $\mathfrak{W}_{1}$ does not have elements of order 16 for $n \leq 8$. So $t^{8}=f^{8}=1$ and $|\mathfrak{G}|$ divides 64 . This rules out the possibility $n=8$, as $64<2^{7}$.

It remains to consider the case $n=6$.

By 2), $\left|\mathfrak{G} \cap \mathfrak{D}_{1}\right|=4,\left|\mathfrak{G} /\left(\mathfrak{G} \cap \mathfrak{D}_{1}\right)\right|=8,|\mathfrak{G}|=32$. Note that $f^{2}$ commutes with $t$. For, $f^{2} t f^{-2}=t^{q^{2}}=t$, as $q^{2} \equiv 1 \bmod 8$.

Let

$$
Y=\left\{e_{1}, \cdots, e_{6},-e_{1}, \cdots,-e_{6}\right\}
$$

which is normalized by $\mathfrak{G}$.

- Then $|I| \neq 4$ :

Assume $|I|=4$. Then $f$ is of order 8 . Either $f$ commutes with $t$ or $f t f^{-1}=t^{-1}$. As $I$ is cyclic, one at least $I$-orbit in $Y$ has 4 elements.

i) Case where exactly 1 I-orbit in $Y$ has 4 elements :

This $I$-orbit, say $O$, is normalized by $f$, and $f$ acts simply transitively on $Y \backslash O=$ : $O^{\prime}$. On $O^{\prime}$, as $t$ and $t^{-1}$ coincide, $t$ commutes with $f$ and thus acts as $f^{4}$ or 1 . If say $O^{\prime}=\left\{e_{1}, \cdots, e_{4},-e_{1}, \cdots,-e_{4}\right\}$, then $\left\{ \pm e_{1} \pm \cdots \pm e_{4}\right\}$ is not acted transitively by $\langle f, t\rangle=\mathfrak{G}$ and thus

$$
X=\left\{s_{1} e_{1}+\cdots+s_{6} e_{6}, s_{i} \in\{1,-1\}, s_{1} \cdots s_{6}=1\right\}
$$

is not acted transitively by $\mathfrak{G}$ either.

ii) Case where exactly 2 I-orbits in $Y$ have 4 elements :

These two, say $O_{1}, O_{2}$, are exchanged by $f$. So $f^{2}$ normalizes and acts as $t$ or $t^{-1}$ on each, since $f^{2}$ commutes with $t$. Both $f^{4}$ and $t^{2}$ act as the identity on $Y \backslash\left(O_{1} \cup O_{2}\right)$. So $f^{4}=t^{2}$. But then $|\mathfrak{G}|$ divides 16 .

iii) Case where exactly 3 I-orbits in $Y$ have 4 elements :

This contradicts the assumption that $t \in \mathfrak{W}_{1}$.

So $|I|=8$. Let $O$ be the unique $I$-orbit of cardinality 8 in $Y$, say

$$
O=\left\{e_{1}, \cdots, e_{4},-e_{1}, \cdots,-e_{4}\right\} .
$$

Let $O^{\prime}:=Y \backslash O$. Then $f$ normalizes $O$ as well as $O^{\prime}$. As $\mathfrak{G}$ acts transitively on $X$, it acts transitively on $\left\{ \pm e_{1} \pm \cdots \pm e_{4}\right\}$. That is, for each choice of $s_{1}, \cdots, s_{4} \in\{1,-1\}$, there are integers $i, j$ such that

$$
s_{1} e_{1}+\cdots+s_{4} e_{4}=f^{i} t^{j}\left(1+t+t^{2}+t^{3}\right) e_{1} .
$$

In particular, there are $i, j \in \mathbf{Z}$ such that

$$
\left(1-t+t^{2}+t^{3}\right) e_{1}=f^{i} t^{j}\left(1+t+t^{2}+t^{3}\right) e_{1} .
$$


Write $f\left(e_{1}\right)=t^{\mu} e_{1}$ for an integer $\mu$. Then $f^{2}=t^{(q+1) \mu}$ on $O$.

- One has $q \not \equiv \pm 1 \bmod 8$ :

For, if $q \equiv 1 \bmod 8$, then

$$
f\left(1+t+t^{2}+t^{3}\right) e_{1}=\left(1+t+t^{2}+t^{3}\right) f\left(e_{1}\right)=t^{\mu}\left(1+t+t^{2}+t^{3}\right) e_{1} .
$$

If $q \equiv-1 \bmod 8$, then

$$
f\left(1+t+t^{2}+t^{3}\right) e_{1}=\left(1+t^{-1}+t^{-2}+t^{-3}\right) f\left(e_{1}\right)=t^{\mu-3}\left(1+t+t^{2}+t^{3}\right) e_{1} .
$$

- The group $I$ acts transitively on $O^{\prime}$ :

Assume that $O^{\prime}$ consists of at least $2 I$-orbits. Choose $x_{1}^{\prime}, x_{2}^{\prime} \in O^{\prime}$ such that $\left(1+t+t^{2}+t^{3}\right) e_{1}+x_{1}^{\prime}+x_{2}^{\prime} \in X$. Now, $t$ is not 1 or -1 on $O^{\prime}$, since

$$
t\left(1+t+t^{2}+t^{3}\right) e_{1}+t x_{1}^{\prime}+t x_{2}^{\prime} \in X .
$$

One may assume $t x_{1}^{\prime}=x_{1}^{\prime}, t x_{2}^{\prime}=-x_{2}^{\prime}$. Then $f$ normalizes $\left\{x_{1}^{\prime},-x_{1}^{\prime}\right\}$ as well as $\left\{x_{2}^{\prime},-x_{2}^{\prime}\right\}$. So $f^{2}$ is the identity on $O^{\prime}$. So $f^{2}=t^{(q+1) \mu}$. But then $|\mathfrak{G}|$ divides 16 .

As now $I$ acts transitively on $O^{\prime}$, there exists $x^{\prime} \in O^{\prime}$ such that $\left(1+t+t^{2}+t^{3}\right) e_{1}+(1+t) x^{\prime} \in X$. One has $f^{2}\left(x^{\prime}\right) \neq t^{(q+1) \mu} x^{\prime}$. For otherwise $f^{2}=t^{(q+1) \mu}$ and $|\mathfrak{G}|$ divides 16 .

- Then $q \not \equiv 3 \bmod 8$ :

Assume $q \equiv 3 \bmod 8$. As $f^{2}\left(x^{\prime}\right) \neq t^{(q+1) \mu} x^{\prime}=x^{\prime}, f$ is of order 4 on $O^{\prime}$. So $f=t$ or $t^{-1}$ on $O^{\prime}$. This contradicts the equation $f t f^{-1}=t^{q}=t^{3}$.

\section{— Then $q \not \equiv 5 \bmod 8$ :}

Assume $q \equiv 5 \bmod 8$. Then $f$ commutes with $t$ on $O^{\prime}$ and so $f=t^{\nu}$ on $O^{\prime}$ for an integer $\nu$. The condition $f^{2}\left(x^{\prime}\right) \neq t^{(q+1) \mu} x^{\prime}$ says that $\nu-\mu$ is an odd integer. But $\nu-\mu$ should also be an even integer. For, the condition that $f$ normalizes $X$ implies that

$$
t^{-\mu} f\left(\left(1+t+t^{2}+t^{3}\right) e_{1}+(1+t) x^{\prime}\right) \in X
$$

which is

$$
\left(1-t+t^{2}-t^{3}\right) e_{1}+(1+t) t^{\nu-\mu} x^{\prime} \in X
$$




\section{TYPE ${ }^{2} D$}

Let $(S, \eta, s)$, $\operatorname{char}(s)=\ell$, be as in $\S 4$.

Suppose given a $\left({ }^{2} D_{n}, \alpha_{1}\right)$ over $\eta$, where $n$ is an integer $\geq 4$. Let

$$
\rho^{2} D_{n}: \pi_{1}(\eta, \bar{\eta}) \rightarrow\{1,-1\}
$$

be the index of ${ }^{2} D_{n}$. One says that ${ }^{2} D_{n}$ is unramified (resp. ramified) over $S$ if its index is unramified (resp. ramified) over $S(\S 4)$.

Write $n=2^{g} r$, for an integer $g \geq 0$ and an odd integer $r \geq 1$.

Let $\mathbf{Z}^{n}$ be identified with $\mathbf{Z}^{2^{g}} \otimes_{\mathbf{Z}} \mathbf{Z}^{r}$ in such a way that the standard basis $e_{1}, \cdots, e_{n}$ of $\mathbf{Z}^{n}$ is identified with $e_{1}^{\prime} \otimes e_{1}^{\prime \prime}, \cdots, e_{2^{g}}^{\prime} \otimes e_{r}^{\prime \prime}$, where $e_{1}^{\prime}, \cdots, e_{2^{g}}^{\prime}$ (resp. $e_{1}^{\prime \prime}, \cdots, e_{r}^{\prime \prime}$ ) denote the standard basis of $\mathbf{Z}^{2^{g}}$ (resp. $\left.\mathbf{Z}^{r}\right)$.

We denote the group of diagonal (resp. monomial) matrices of $\mathrm{GL}_{n}(\mathbf{Z})$ by $\mathfrak{D}($ resp. $\mathfrak{M})$. Let $\mathfrak{D}_{1}$ be the subgroup of $\mathfrak{D}$ consisting of all diagonal matrices of determinant 1 .

Let $\mathfrak{W}=\mathfrak{D M}$ and $\mathfrak{W}_{1}=\mathfrak{D}_{1} \mathfrak{M}$.

Proposition 10.1. Suppose that ${ }^{2} D_{n}$ is unramified over $S$. Then $\left({ }^{2} D_{n}, \alpha_{1}\right)$ is elliptic.

Proof. Let $\zeta, \tau \in \mathfrak{W}$ be such that

$$
\begin{gathered}
\zeta: e_{1} \mapsto e_{2}, \cdots, e_{n} \mapsto e_{1} \\
\tau: e_{1} \mapsto-e_{1}, e_{i} \mapsto e_{i}, \forall i>1 .
\end{gathered}
$$

The cyclic group $\mathfrak{G}$ generated by $\tau \zeta$ acts simply transitively on

$$
\left\{e_{1}, \cdots, e_{n},-e_{1}, \cdots,-e_{n}\right\} .
$$

Choose a surjective homomorphism $(\S 4)$ :

$$
\rho: \pi_{1}(\eta, \bar{\eta}) \rightarrow \pi_{1}(S, \bar{\eta}) \rightarrow \mathbf{Z} / 2 n \mathbf{Z}=\mathfrak{G}
$$

The composition

$$
\pi_{1}(\eta, \bar{\eta}) \stackrel{\rho}{\longrightarrow} \mathfrak{G} \hookrightarrow \mathfrak{W} \rightarrow \mathfrak{W} / \mathfrak{W}_{1}=\{1,-1\}
$$

is the index of ${ }^{2} D_{n}$, as ${ }^{2} D_{n}$ is unramified over $S$. So $\left({ }^{2} D_{n}, \alpha_{1}\right)$ is elliptic $(3.1), 9)$.

Proposition 10.2. Suppose $\ell>2$ and that ${ }^{2} D_{n}$ is ramified over $S$. Then $\left({ }^{2} D_{n}, \alpha_{1}\right)$ is elliptic. 
Proof. As $\ell>2$, the index of ${ }^{2} D_{n}$ is tamely ramified over $S$ :

$$
\rho^{2} D_{n}: \pi_{1}(\eta, \bar{\eta}) \rightarrow \pi_{1}^{t}(\eta, \bar{\eta})=\langle F, T\rangle \stackrel{\bar{\rho}}{\longrightarrow}\{1,-1\}, \bar{\rho}: T \mapsto-1 .
$$

Let $q=\operatorname{Card}(k(s))$. Let $\tau^{\prime}, \sigma^{\prime} \in \mathrm{GL}_{2^{g}}(\mathbf{Z})$ be such that

$$
\begin{gathered}
\tau^{\prime}: e_{1}^{\prime} \mapsto e_{2}^{\prime}, \cdots, e_{2^{g}-1}^{\prime} \mapsto e_{2^{g}}^{\prime}, e_{2^{g}}^{\prime} \mapsto-e_{1}^{\prime}, \\
\sigma^{\prime} \tau^{\prime}=\tau^{q^{r}} \sigma^{\prime}, \quad \sigma^{\prime}: e_{1}^{\prime} \mapsto e_{1}^{\prime} .
\end{gathered}
$$

Let $\tau \in \mathrm{GL}_{n}(\mathbf{Z})$ be such that

$$
\tau: e_{i}^{\prime} \otimes e_{j}^{\prime \prime} \mapsto \tau^{\prime q^{j-1}}\left(e_{i}^{\prime}\right) \otimes e_{j}^{\prime \prime}, \forall j=1, \cdots, r, \forall i=1, \cdots, 2^{g} .
$$

And let $\sigma \in \mathrm{GL}_{n}(\mathbf{Z})$ be such that

$$
\sigma: e_{i}^{\prime} \otimes e_{1}^{\prime \prime} \mapsto e_{i}^{\prime} \otimes e_{2}^{\prime \prime}, \cdots, e_{i}^{\prime} \otimes e_{r-1}^{\prime \prime} \mapsto e_{i}^{\prime} \otimes e_{r}^{\prime \prime}, e_{i}^{\prime} \otimes e_{r}^{\prime \prime} \mapsto \sigma^{\prime}\left(e_{i}^{\prime}\right) \otimes e_{1}^{\prime \prime}
$$

$\forall i=1, \cdots, 2^{g}$. Then $\tau$ is of order $2^{g+1}, \sigma^{r}=\sigma^{\prime} \otimes 1$ and $\sigma \tau \sigma^{-1}=\tau^{q}$.

The subgroup $\mathfrak{G}$ of $\mathfrak{W}$ generated by $\{\sigma, \tau\}$ acts transitively on

$$
\left\{e_{1}, \cdots, e_{n},-e_{1}, \cdots,-e_{n}\right\} \text {. }
$$

Let

$$
\rho: \pi_{1}^{t}(\eta, \bar{\eta}) \rightarrow \mathfrak{G}, T \mapsto \tau
$$

which maps $F$ to :

$-\sigma$, if $\sigma \in \mathfrak{W}_{1}, \bar{\rho}: F \mapsto 1$.

$-\sigma \tau$, if $\sigma \in \mathfrak{W}_{1}, \bar{\rho}: F \mapsto-1$.

- $\sigma \tau$, if $\sigma \notin \mathfrak{W}_{1}, \bar{\rho}: F \mapsto 1$.

Then the composition

$$
\pi_{1}(\eta, \bar{\eta}) \stackrel{\rho}{\longrightarrow} \mathfrak{G} \hookrightarrow \mathfrak{W} \rightarrow \mathfrak{W} / \mathfrak{W}_{1}=\{1,-1\}
$$

is the index of ${ }^{2} D_{n}$. So $\left({ }^{2} D_{n}, \alpha_{1}\right)$ is elliptic $\left.(3.1), 9\right)$.

Let $d$ (resp. $f$ ) be an integer $\geq 1$ (resp. $>1$ ). Let pro-2-groups $F_{1}, F_{2}, F_{3}, F_{4}$ be defined by generators and relations as:

$$
\begin{gathered}
\left.F_{1}=\left\langle x_{1}, \cdots, x_{d+2}\right| x_{1}^{2^{f}}\left[x_{1}, x_{2}\right]\left[x_{3}, x_{4}\right] \cdots\left[x_{d+1}, x_{d+2}\right]=1, d \text { even }\right\rangle, \\
\left.F_{2}=\left\langle x_{1}, \cdots, x_{d+2}\right| x_{1}^{2} x_{2}^{4}\left[x_{2}, x_{3}\right] \cdots\left[x_{d+1}, x_{d+2}\right]=1, d \text { odd }\right\rangle, \\
\left.F_{3}=\left\langle x_{1}, \cdots, x_{d+2}\right| x_{1}^{2+2^{f}}\left[x_{1}, x_{2}\right]\left[x_{3}, x_{4}\right] \cdots\left[x_{d+1}, x_{d+2}\right]=1, d \text { even }\right\rangle, \\
\left.F_{4}=\left\langle x_{1}, \cdots, x_{d+2}\right| x_{1}^{2}\left[x_{1}, x_{2}\right] x_{3}^{2^{f}}\left[x_{3}, x_{4}\right] \cdots\left[x_{d+1}, x_{d+2}\right]=1, d \text { even }\right\rangle,
\end{gathered}
$$

where

$$
x, y \mapsto[x, y]=x^{-1} y^{-1} x y
$$

denotes the commutator. 
When $\ell=2, \pi_{1}(\eta, \bar{\eta})$ has one of the groups $F_{1}, F_{2}, F_{3}, F_{4}$ as the maximal pro-2-quotient, for $d=\left[\eta: \mathbf{Q}_{2}\right]$ and for a certain integer $f$ ([1], p. 107-108).

Proposition 10.3. Suppose $\ell=2$. Then $\left({ }^{2} D_{n}, \alpha_{1}\right)$ is elliptic.

Proof. Let $a^{\prime}, b^{\prime} \in \mathrm{GL}_{2^{g}}(\mathbf{Z})$ be such that

$$
\begin{gathered}
a^{\prime}: e_{1}^{\prime} \mapsto-e_{1}^{\prime}, e_{i}^{\prime} \mapsto e_{i}^{\prime}, \forall i>1, \\
b^{\prime}: e_{1}^{\prime} \mapsto e_{2}^{\prime}, \cdots, e_{2^{g}-1}^{\prime} \mapsto e_{2^{g}}^{\prime}, e_{2^{g}}^{\prime} \mapsto e_{1}^{\prime} .
\end{gathered}
$$

Let $c^{\prime \prime} \in \mathrm{GL}_{r}(\mathbf{Z})$ be such that

$$
c^{\prime \prime}: e_{1}^{\prime \prime} \mapsto e_{2}^{\prime \prime}, \cdots, e_{r-1}^{\prime \prime} \mapsto e_{r}^{\prime \prime}, e_{r}^{\prime \prime} \mapsto e_{1}^{\prime \prime} .
$$

Let $a=a^{\prime} \otimes 1, b=b^{\prime} \otimes 1, c=1 \otimes c^{\prime \prime} \in \mathfrak{W}$.

Notice that $a$ (resp. $b$, resp. $c$ ) has image -1 (resp. 1, resp. 1) by

$$
\mathfrak{W} \rightarrow \mathfrak{W} / \mathfrak{W}_{1}=\{1,-1\} .
$$

The group $\langle a b\rangle \times\langle c\rangle$ acts simply transitive on

$$
\left\{e_{1}, \cdots, e_{n},-e_{1}, \cdots,-e_{n}\right\} .
$$

By $(3.1), 9)$ it suffices to show that either $\langle a b\rangle \times\langle c\rangle$ or $\langle a, b\rangle \times\langle c\rangle$ is realizable as a quotient of $\pi_{1}(\eta, \bar{\eta})$ lifting the index of ${ }^{2} D_{n}$. Now, this index factors through the maximal pro-2-quotient $F$ of $\pi_{1}(\eta, \bar{\eta})$ :

$$
\pi_{1}(\eta, \bar{\eta}) \rightarrow F \stackrel{\chi}{\longrightarrow}\{1,-1\} .
$$

And, the odd order cyclic subgroup $\langle c\rangle$ of $\mathfrak{W}_{1}$ is realizable as an unramified quotient of $\pi_{1}(\eta, \bar{\eta})$. So it suffices to show that every surjective homomorphism

$$
\chi: F \rightarrow\{1,-1\}
$$

is a composition of the form

$$
F \stackrel{\rho}{\longrightarrow}\langle a, b\rangle \hookrightarrow \mathfrak{W} \rightarrow \mathfrak{W} / \mathfrak{W}_{1}=\{1,-1\}
$$

for some representation

$$
\rho: F \rightarrow\langle a, b\rangle
$$

whose image is $\langle a b\rangle$ or $\langle a, b\rangle$.

Given the explicit structure of $F$ as above, the verification is straightforward. Consider for example the case where

$$
F=\left\langle x, y, z \mid x^{2} y^{4}[y, z]=1\right\rangle
$$

and where $g \geq 2$. According to the values of $\chi$ on $(x, y, z)$, one defines $\rho: F \rightarrow\langle a, b\rangle$ as follows :

1) $(-1,1,1)$. Let $\rho:(x, y, z) \mapsto(a, 1, b)$.

2) $(1,-1,1)$. Let $\rho:(x, y, z) \mapsto\left((a b)^{-2}, a b, 1\right)$. 
3) $(1,1,-1)$. Let $\rho:(x, y, z) \mapsto(1,1, a b)$.

4) $(-1,1,-1)$. Let $\rho:(x, y, z) \mapsto(a, 1, a b)$.

5) $(1,-1,-1)$. Let $\rho:(x, y, z) \mapsto\left((a b)^{-2}, a b, a b\right)$.

$6)(-1,-1,1)$. Let $\rho:(x, y, z) \mapsto\left(a, a b, a b^{2} a b^{-2}\right)$, if $g=2$, and let $\rho:(x, y, z) \mapsto\left(a b^{2}, a b^{-1}, a b^{3} a b^{-3}\right)$, if $g>2$.

7) $(-1,-1,-1)$. Let $\rho:(x, y, z) \mapsto\left(a b^{2}, a b, a b^{-1}\right)$, if $g=2$, and let $\rho:(x, y, z) \mapsto\left(b^{-1} a b^{2} a b a, a b^{-1}, a b\right)$, if $g>2$.

\section{TyPE $E_{6}$}

Let $E$ be a 6 -dimensional $\mathbf{F}_{2}$-vector space. Let $e_{i}, f_{j}, 1 \leq i, j \leq 3$, be a basis of $E$ and let $q$ be the quadratic form on $E$ such that

$$
q\left(e_{i}\right)=q\left(f_{j}\right)=1, q\left(e_{i}+e_{j}\right)=q\left(f_{i}+f_{j}\right)=0, q\left(e_{i}+f_{j}\right)=\delta_{i j},
$$

where $\delta_{i j}=1$, if $i=j$, and $\delta_{i j}=0$, if $i \neq j, \forall i, j \in\{1,2,3\}$.

Let

$$
X=\{v \in E \backslash\{0\}, q(v)=0\}
$$

be the $q$-singular vectors of $E \backslash\{0\}$.

Let $V_{i}=\mathbf{F}_{2} e_{i}+\mathbf{F}_{2} f_{i}, i=1,2,3$. The elements of $X$ are of the form $v_{i}+v_{j}$, where $v_{i} \in V_{i}, v_{j} \in V_{j}, 1 \leq i, j \leq 3, i \neq j, v_{i}, v_{j} \neq 0$. The set $X$ consists of 27 vectors which are permuted transitively by the orthogonal group $\mathrm{O}(q)$. The group $\mathrm{O}(q)$ has $2^{7} .3^{4} .5$ elements.

Observe that an element of $\mathrm{GL}(E)$ belongs to $\mathrm{O}(q)$ if and only if it normalizes $X$.

Note that, for each $i \in\{1,2,3\}$, one has $\mathrm{O}\left(q \mid V_{i}\right)=\mathrm{GL}\left(V_{i}\right)$, because $q\left(e_{i}\right)=q\left(f_{i}\right)=q\left(e_{i}+f_{i}\right)=1$. The subgroup $\mathrm{GL}\left(V_{1}\right) \times \mathrm{GL}\left(V_{2}\right) \times \mathrm{GL}\left(V_{3}\right)$ of $\mathrm{O}(q)$ consists of all elements $g$ such that $g\left(V_{1}\right)=V_{1}, g\left(V_{2}\right)=V_{2}$, $g\left(V_{3}\right)=V_{3}$.

Let $\mathfrak{N}$ be the subgroup of $\mathrm{O}(q)$ consisting of all elements $g$ such that $g\left(V_{i}\right) \in\left\{V_{1}, V_{2}, V_{3}\right\}, \forall i=1,2,3$. One has a split exact sequence

$$
1 \rightarrow \prod_{1}^{3} \mathrm{GL}\left(V_{i}\right) \rightarrow \mathfrak{N} \rightarrow \operatorname{Aut}\left(\left\{V_{1}, V_{2}, V_{3}\right\}\right) \rightarrow 1
$$

The unique 3-Sylow subgroup $\mathfrak{M}$ of $\prod_{i} \mathrm{GL}\left(V_{i}\right)$ is the unique abelian subgroup of order 27 of $\mathfrak{N}$.

Lemma 11.1. Suppose that a solvable subgroup $\mathfrak{G}$ of $\mathrm{O}(q)$ acts transitively on $X$. Then $5 \nmid|\mathfrak{G}|$. 
Proof. Let $\mathfrak{H}$ be a Hall subgroup of $\mathfrak{G}$ which is a product of a 3-Sylow subgroup and a 5-Sylow subgroup of $\mathfrak{G}$. Then $\mathfrak{H}$ also acts transitively on $X$.

- Case $3^{4}$ does not divide $|\mathfrak{H}|$ :

Then $\mathfrak{H}$ has a unique 5-Sylow subgroup, say $\mathfrak{Q}$. The $\mathfrak{Q}$-orbits in $X$ all have the same cardinality, say $r$, which divides both 5 and $\operatorname{Card}(X)=$ 27. So $r=1$. So $\mathfrak{Q}=1$. For, if an element $g \in \mathrm{GL}(E)$ restricts to the identity on $X$, then $g=1$.

- Case $3^{4}$ divides $|\mathfrak{H}|$ :

Then $\mathfrak{H}$ has a unique 3-Sylow subgroup $\mathfrak{P}$ which one may, by conjugating $\mathfrak{H}$ in $\mathrm{O}(q)$, assume to be in $\mathfrak{N}$. In particular, $\mathfrak{P}$ contains $\mathfrak{M}$. Let $\mathfrak{Q}$ be a 5-Sylow subgroup of $\mathfrak{H}$. Then $\mathfrak{Q}$ normalizes and thus centralizes $\mathfrak{M}$. Notice that $\mathfrak{M}$ has 3 -orbits in $X$ each of which consists of 3 elements. The group $\mathfrak{Q}$ normalizes each of these 3 -orbits and so it fixes every point of $X$. So $\mathfrak{Q}=1$.

Lemma 11.2. Suppose that a solvable subgroup $\mathfrak{G}$ of $\mathrm{O}(q)$ acts transitively on $X$. Let $\mathfrak{A}$ be an abelian normal subgroup of $\mathfrak{G}$. Then $\mathfrak{A}$ is a 3-group.

Proof. Let $\mathfrak{Q}$ be the unique 2-Sylow subgroup of $\mathfrak{A}$. The group $\mathfrak{Q}$ is normal in $\mathfrak{G}$. So the $\mathfrak{Q}$-orbits in $X$ all have the same cardinality, say $r$, which divides both $|\mathfrak{Q}|$ and $27=\operatorname{Card}(X)$. So $r=1$. So $\mathfrak{Q}=1$. By (11.1), the lemma follows.

Proposition 11.3. Let $\mathfrak{H}$ be a cyclic subgroup of order 9 of $\mathrm{O}(q)$. Let $h$ be a generator of $\mathfrak{H}$.

Then the commutant of $\mathfrak{H}$ on $E$ is a field of cardinality 64 . And $\mathfrak{H}$ has 3 orbits on $X$ each of which consists of 9 points. Let $x, y \in X$ be in distinct $\mathfrak{H}$-orbits. Then there exists a unique element $g \in \mathrm{O}(q)$ of order 6 which satisfies the following properties :

$$
g h g^{-1}=h^{2}, g(x)=y .
$$

The group $\mathfrak{G}$ generated by $\{h, g\}$ acts transitively on $X$ and $\mathfrak{G}$ is of order 54 .

Proof. Notice that $\left[\mathbf{Q}\left(\mu_{9}\right): \mathbf{Q}\right]=6$ and that 2 is inert in $\mathbf{Q}\left(\mu_{9}\right)$. So $\mathfrak{H}$ acts irreducibly on $E$ and the commutant $C$ of $\mathfrak{H}$ on $E$ is a field of cardinality 64. In particular, neither $h$ nor $h^{3}$ fixes a nonzero vector in 
$E$, as $E$ is a 1 -dimensional $C$-vector space. Every $\mathfrak{H}$-orbit in $X$ consists of 9 points.

Let $F: C \rightarrow C, c \mapsto c^{2}$, be the Frobenius automorphism of $C$. An element $g \in \mathrm{GL}(E)$ satisfying $g h g^{-1}=h^{2}$ is simply an $F$-linear automorphism of the 1-dimensional $C$-vector space $E$. Let $g$ be an $F$-linear automorphism of $E$ and let $x \in E$ be a nonzero vector. For every integer $n, g^{n}$ is $F^{n}$-linear. So $g$ is of order a multiple of 6 . Write $g(x)=c . x$ for an element $c \in C^{\times}$. As $g^{6}$ is $C$-linear and as

$$
g^{6}(x)=F^{5}(c) \cdots F(c) c . x=c^{63} \cdot x=x,
$$

$g$ is of order 6 .

Note finally that $g$ lies in $\mathrm{O}(q)$ if and only if it normalizes $X$. From here, the claimed existence and uniqueness of $g$ as well as the last assertion immediately follow.

Proposition 11.4. Suppose that a solvable subgroup $\mathfrak{G}$ of $\mathrm{O}(q)$ acts transitively on $X$. Suppose furthermore that $\mathfrak{G}$ has a cyclic normal subgroup $\mathfrak{H}$ of generator $h$ of order 9 . Then $|\mathfrak{G}|=27$ or 54 .

- Case $|\mathfrak{G}|=27$. Then $\mathfrak{G}$ is generated by $\{h, g\}$ where the element $g$ is of order 3 and satisfies $\mathrm{ghg}^{-1}=h^{4}$.

- Case $|\mathfrak{G}|=54$. Then $\mathfrak{G}$ is generated by $\{h, g\}$ where the element $g$ is of order 6 and satisfies $g h g^{-1}=h^{2}$.

Proof. Let $C$ be the commutant of $\mathfrak{H}$ on $E$. By (11.3), $C$ is a field of cardinality 64 and $E$ is a 1 -dimensional $C$-vector space. The centralizer of $\mathfrak{H}$ in $\mathfrak{G}$ is the intersection $\mathfrak{G} \cap C^{\times}$, that is, $\mathfrak{H}$. Now, the exact sequence

$$
1 \rightarrow \mathfrak{H} \rightarrow \mathfrak{G} \stackrel{g \mapsto \operatorname{Int}(g) \mid \mathfrak{H}}{\longrightarrow} \operatorname{Aut}(\mathfrak{H})
$$

shows that $\mathfrak{G}$ is of order 27 or 54 , as $\operatorname{Aut}(\mathfrak{H})$ is cyclic of order 6 . Choose $g \in \mathfrak{G}$ such that $\operatorname{Int}(g) \mid \mathfrak{H}$ generates $\operatorname{Int}(\mathfrak{G}) \mid \mathfrak{H}$. The automorphism $\operatorname{Int}(g) \mid \mathfrak{H}$ extends to an automorphism of the field $C$.

- Case where $|\mathfrak{G}|=27$ :

Replacing if necessary $g$ by its inverse, one may assume $g h g^{-1}=h^{4}$. As GL $(E)$ has no element of order 27 , one has $g^{3}=h^{3 n}$ for some integer $n$. The group $\mathfrak{G}$ is generated by $\left\{h, g h^{-n}\right\}$ and $\left(g h^{-n}\right)^{3}=\left(g h^{-n} g^{-1}\right)\left(g^{2} h^{-n} g^{-2}\right)\left(g^{3} h^{-n} g^{-3}\right) g^{3}=h^{-4 n} h^{-16 n} h^{-64 n} g^{3}=1$.

- Case where $|\mathfrak{G}|=54$ :

Replacing if necessary $g$ by its inverse, one may assume $g h g^{-1}=h^{2}$. As in (11.3), $g$ is of order 6 . And $\mathfrak{G}$ is generated by $\{h, g\}$. 
Proposition 11.5. Let $(S, \eta, s)$, $\operatorname{char}(k(s))=\ell$, be as in $\S 4$. Suppose that $\ell=3$. Then $\left(E_{6}, \alpha_{1}\right)$ and $\left(E_{6}, \alpha_{6}\right)$ are elliptic over $\eta$.

Proof. The orthogonal group $\mathrm{O}(q)$ has a subgroup $\mathfrak{G}$ of order 27 which acts transitively on $X$ and which is generated by 2 elements $h, g$, where $h$ (resp. $g$ ) is of order 9 (resp. 3) and $g h g^{-1}=h^{4}$.

- Case where $\mu_{3}(k(\eta))=1$ :

In this case, the maximal pro-3-quotient of $\pi_{1}(\eta, \bar{\eta})$ is free of rank $\geq 2$ as a pro-3-group. In particular, $\mathfrak{G}$ is realizable as a quotient of $\pi_{1}(\eta, \bar{\eta})$. So $\left(E_{6}, \alpha_{1}\right)$ and $\left(E_{6}, \alpha_{6}\right)$ are elliptic over $\left.\eta(3.1), 6\right)$.

- Case where $\mu_{3}(k(\eta))=\mu_{3}(k(\bar{\eta}))$ :

The maximal pro-3-quotient of $\pi_{1}(\eta, \bar{\eta})$ has then the presentation :

$$
F=\left\langle x_{1}, \cdots, x_{d+2} \mid x_{1}^{q}\left[x_{1}, x_{2}\right]\left[x_{3}, x_{4}\right] \cdots\left[x_{d+1}, x_{d+2}\right]=1\right\rangle
$$

where $d=\left[k(\eta): \mathbf{Q}_{3}\right]$, where $q$ is the maximal power of 3 such that $\mu_{q}(k(\eta))=\mu_{q}(k(\bar{\eta}))$ and where $(x, y) \mapsto[x, y]=x^{-1} y^{-1} x y$ is the commutator. The homomorphism $\chi: F \rightarrow \mathfrak{G}$ such that

$$
\chi: x_{1} \mapsto 1, x_{2} \mapsto h, x_{3} \mapsto g, x_{i} \mapsto 1, \forall i>3
$$

is surjective. So again $\left(E_{6}, \alpha_{1}\right)$ and $\left(E_{6}, \alpha_{6}\right)$ are elliptic over $\left.\eta(3.1), 6\right)$.

Proposition 11.6. Let $(S, \eta, s)$, $\operatorname{char}(k(s))=\ell$, be as in $\S 4$. Suppose $\ell \neq 3$. Then $\left(E_{6}, \alpha_{1}\right)$ and $\left(E_{6}, \alpha_{6}\right)$ are elliptic over $\eta$ if and only if $\operatorname{Card}(k(s)) \equiv \pm 2, \pm 4 \bmod 9$.

Proof. By $(3.1), 6)$, the pairs $\left(E_{6}, \alpha_{1}\right)$ and $\left(E_{6}, \alpha_{6}\right)$ are elliptic over $\eta$ if and only if there is a representation

$$
\rho: \pi_{1}(\eta, \bar{\eta}) \rightarrow \mathrm{O}(q)
$$

whose image acts transitively on $X$. Suppose that such a representation exists. Let $\mathfrak{G}$ be its image. Let $I$ (resp. $P$ ) be the image in $\mathfrak{G}$ of the inertia (resp. wild inertia) subgroup of $\pi_{1}(\eta, \bar{\eta})$.

As $P$ is normal in $\mathfrak{G}$, the $P$-orbits in $X$ all have the same cardinality, say $r$, which divides both 27 and $|P|$. That is, $r=1$ and $P=1$.

So $I=I / P$ is cyclic of order a power of $3(11.2)$ and so $\mathfrak{G}$ has a unique 3 -Sylow subgroup, say $\mathfrak{H}$. As $\mathfrak{H} / I$ is cyclic, the group $I$ is cyclic of order 9 and $\mathfrak{H} / I$ is of order 3 . The quotient $\mathfrak{G} / \mathfrak{H}$ is a cyclic 2 -group (11.1). So $|\mathfrak{G}|=27$ or 54 (11.4). Write $\rho$ as a composition

$$
\pi_{1}(\eta, \bar{\eta}) \rightarrow \pi_{1}^{t}(\eta, \bar{\eta}) \stackrel{\chi}{\longrightarrow} \mathfrak{G},
$$

where $\pi_{1}^{t}(\eta, \bar{\eta})=\langle F, T\rangle(\S 4)$. The image $t$ of $T$ in $\mathfrak{G}$ generates $I$. Let $f$ be the image of $F$ in $\mathfrak{G}$. 
- Case where $|\mathfrak{G}|=54$ :

Then $f$ is of order 6 . One has $f t f^{-1}=t^{2}$ or $t^{32}=t^{-4}$.

- Case where $|\mathfrak{G}|=27$ :

Then $f$ is of order 3 or 9 . One has $f t f^{-1}=t^{4}$ or $t^{16}=t^{-2}$.

Such groups do exist in $\mathrm{O}(q)(11.3)$

\section{TYPE $E_{7}$}

Let $E$ be a 6 -dimensional $\mathbf{F}_{2}$-vector space equipped with a symplectic form $($,$) . Let e_{i}, f_{j}, 1 \leq i, j \leq 3$, be a sympletic base of $E$. Let $q$ be the quadratic form on $E$ satisfying

$$
q\left(e_{i}\right)=q\left(f_{j}\right)=1, q\left(e_{i}+e_{j}\right)=q\left(f_{i}+f_{j}\right)=0, q\left(e_{i}+f_{j}\right)=\delta_{i j}
$$

where $\delta_{i j}=1$, if $i=j$, and $\delta_{i j}=0$, if $i \neq j, \forall i, j \in\{1,2,3\}$.

Observe that the orthogonal group $\mathrm{O}(q)$ is a subgroup of the symplectic group $\operatorname{Sp}(E)$.

The group $\operatorname{Sp}(E)$ is of order $2^{9} .3^{4} .5 .7$, the subgroup $\mathrm{O}(q)$ is of order $2^{7} .3^{4} .5$ and the homogenous space

$$
X=\operatorname{Sp}(E) / \mathrm{O}(q)
$$

consists of 28 elements.

We shall determine up to conjugation all solvable subgroups $\mathfrak{G}$ of $\operatorname{Sp}(E)$ that act transitively on $X$.

Each such group $\mathfrak{G}$ contains a 7-Sylow subgroup of $\operatorname{Sp}(E)$. By conjugation in $\operatorname{Sp}(E)$, one may suppose that $\mathfrak{G}$ contains $\zeta \in \operatorname{Sp}(E)$, where

$$
\zeta:\left\{\begin{array}{l}
e_{1} \mapsto e_{2}, e_{2} \mapsto e_{3}, e_{3} \mapsto e_{1}+e_{2} \\
f_{1} \mapsto f_{1}+f_{2}, f_{2} \mapsto f_{3}, f_{3} \mapsto f_{1}
\end{array}\right.
$$

Let $V=\mathbf{F}_{2} e_{1}+\mathbf{F}_{2} e_{2}+\mathbf{F}_{2} e_{3}, V^{\vee}=\mathbf{F}_{2} f_{1}+\mathbf{F}_{2} f_{2}+\mathbf{F}_{2} f_{3}$. Then

$$
\operatorname{det}(T-\zeta, V)=T^{3}+T+1, \operatorname{det}\left(T-\zeta, V^{\vee}\right)=T^{3}+T^{2}+1
$$

and

$$
\operatorname{det}(T-\zeta, E)=\left(T^{3}+T+1\right)\left(T^{3}+T^{2}+1\right)=\left(T^{7}-1\right) /(T-1) .
$$

As $\zeta$-modules, $V, V^{\vee}$ are irreducible mutually non-isomorphic. The subspaces $0, V, V^{\vee}, E$ are the only sub- $\zeta$-modules of $E$.

The commutant $\operatorname{End}_{\zeta}(E)$ is equal to $\mathbf{F}_{2}[\zeta \mid V] \times \mathbf{F}_{2}\left[\zeta \mid V^{\vee}\right]$. And

$$
\mathrm{GL}_{\zeta}(E) \cap \mathrm{Sp}(E)=\mathbf{F}_{2}[\zeta]^{\times}=\langle\zeta\rangle \text {. }
$$

That is, $\langle\zeta\rangle$ is its own centralizer in $\operatorname{Sp}(E)$. 
The normalizer of $\langle\zeta\rangle$ in $\operatorname{Sp}(E)$ admits 2 generators $\zeta, \sigma$, where

$$
\sigma:\left\{\begin{array}{l}
e_{1} \mapsto f_{1}, e_{2} \mapsto f_{2}, e_{3} \mapsto f_{2}+f_{3} \\
f_{1} \mapsto e_{1}, f_{2} \mapsto e_{2}+e_{3}, f_{3} \mapsto e_{3}
\end{array}\right.
$$

And $\sigma, \zeta$ satisfy the relations :

$$
\sigma^{6}=1, \sigma \zeta \sigma^{-1}=\zeta^{-2}
$$

Notice that $|\langle\zeta, \sigma\rangle|=42$.

Let $\mathfrak{S}$ be the subgroup of $\operatorname{Sp}(E)$ consisting of all elements which act as the identity on $V$. By $g \mapsto(g-1) \mid V^{\vee}, \mathfrak{S}$ can be identified with an $\mathbf{F}_{2}$-vector space of dimension 6 which consists of all linear transformations $A: V^{\vee} \rightarrow V$ such that the bilinear form

$$
u^{\prime}, v^{\prime} \mapsto\left(u^{\prime}, A v^{\prime}\right)
$$

is symmetric in $u^{\prime}, v^{\prime} \in V^{\vee}$.

For all $g \in \mathfrak{S}$, the function $v^{\prime} \mapsto\left(v^{\prime},(g-1) v^{\prime}\right)$ is linear on $V^{\vee}$. Thus there is a unique vector $v_{g} \in V$ satisfying

$$
\left(v^{\prime},(g-1) v^{\prime}\right)=\left(v_{g}, v^{\prime}\right), \quad \forall v^{\prime} \in V^{\vee} .
$$

The function $\mathfrak{S} \rightarrow V, g \mapsto v_{g}$, is linear whose kernel $\mathfrak{S}^{1}$ consists of all those $g \in \mathfrak{S}$ such that the form

$$
u^{\prime}, v^{\prime} \mapsto\left(u^{\prime},(g-1) v^{\prime}\right)
$$

is alternating, i.e., that

$$
\left(u^{\prime},(g-1) v^{\prime}\right)=\left(u^{\prime} \wedge v^{\prime}, \omega_{g}\right)
$$

for a uniquely determined 2-form $\omega_{g} \in \wedge^{2} V$.

The map $g \mapsto \omega_{g}$ establishes a canonical bijection between $\mathfrak{S}^{1}$ and $\wedge^{2} V$. The exact sequence

$$
0 \rightarrow \mathfrak{S}^{1} \rightarrow \mathfrak{S} \stackrel{g \mapsto v_{g}}{\longrightarrow} V \rightarrow 0
$$

is uniquely split as $\zeta$-modules. For, $\wedge^{2} V=\mathfrak{S}^{1}$ and $V$ are non-isomorphic $\zeta$-modules. Let $\mathfrak{S}^{2}$ denote this complement of $\mathfrak{S}^{1}$ in $\mathfrak{S}$. So $\mathfrak{S}=$ $\mathfrak{S}^{1} \oplus \mathfrak{S}^{2}$.

In terms of matrices, every element $g \in \mathfrak{S}$ is of the form

$$
g:\left\{\begin{array}{l}
e_{i} \mapsto e_{i}, i=1,2,3 \\
f_{i} \mapsto f_{i}+\sum_{j=1,2,3} A_{j i} e_{j}
\end{array}\right.
$$

where $A_{i j}$ is a symmetric matrix with coefficients in $\mathbf{F}_{2}$.

The element $g$ belongs to $\mathfrak{S}^{1}$ if and only if $A_{11}=A_{22}=A_{33}=0$. The $\zeta$-module $\mathfrak{S}^{1}$ is generated by $g_{1}$, where

$$
g_{1}:\left\{\begin{array}{l}
e_{i} \mapsto e_{i}, i=1,2,3 \\
f_{1} \mapsto f_{1}+e_{2}+e_{3}, f_{2} \mapsto f_{2}+e_{1}+e_{3}, f_{3} \mapsto f_{3}+e_{1}+e_{2}
\end{array}\right.
$$


The $\zeta$-module $\mathfrak{S}^{2}$ is generated by $g_{2}$, where

$$
g_{2}:\left\{\begin{array}{l}
e_{i} \mapsto e_{i}, i=1,2,3 \\
f_{1} \mapsto f_{1}+e_{2}+e_{3}, f_{2} \mapsto f_{2}+e_{1}+e_{3}, f_{3} \mapsto f_{3}+e_{1}+e_{2}+e_{3}
\end{array}\right.
$$

The element $g \in \mathfrak{S}$ preserves the quadratic form $q$ if and only if $A_{12}=A_{23}=A_{13}$. One has $\mathfrak{S}^{1} \cap \mathrm{O}(q)=\left\{1, g_{1}\right\}$ and $\mathfrak{S}^{2} \cap \mathrm{O}(q)=\left\{1, g_{2}\right\}$.

Proposition 12.1. Up to conjugation all solvable subgroups of $\operatorname{Sp}(E)$ that act transitively on $X$ are enumerated as follows :

$-\langle\zeta\rangle \mathfrak{S}$.

$-\left\langle\zeta, \sigma^{2}\right\rangle \mathfrak{S}$.

$-\langle\zeta\rangle \mathfrak{S}^{1},\langle\zeta\rangle \mathfrak{S}^{2}$.

$-\left\langle\zeta, \sigma^{2}\right\rangle \mathfrak{S}^{1},\left\langle\zeta, \sigma^{2}\right\rangle \mathfrak{S}^{2}$.

Proof. Suppose that $\mathfrak{G}$ is a solvable subgroup of $\operatorname{Sp}(E)$ which acts transitively on $X$. Up to conjugation in $\operatorname{Sp}(E)$ one may assume that $\zeta \in \mathfrak{G}$. Recall that $|\operatorname{Sp}(E)|=2^{9} .3^{4} .5 .7,|\mathrm{O}(q)|=2^{7} .3^{4} .5$.

- Then $5 \nmid|\mathfrak{G}|$ :

Otherwise, as it is solvable, $\mathfrak{G}$ has a Hall subgroup of order 35 , say $\mathfrak{Q}$, which is cyclic. But $\mathbf{Z} / 35 \mathbf{Z}$ admits no faithful 6-dimensional representations over $\mathbf{F}_{2}$.

Thus $|\mathfrak{G}|=2^{a} .3^{b} .7$, for an integer $a \geq 2$ and an integer $0 \leq b \leq 4$.

Let $\mathfrak{L}$ be a Hall subgroup of $\mathfrak{G}$ which is a product of $\langle\zeta\rangle$ and a 3Sylow subgroup of $\mathfrak{G}$. As $b \leq 4,\langle\zeta\rangle$ is normal in $\mathfrak{L}$. So $\mathfrak{L}$ is a subgroup of $\langle\zeta, \sigma\rangle$. So $\mathfrak{L}=\langle\zeta\rangle$ or $\left\langle\zeta, \sigma^{2}\right\rangle$. In particular, $b=0$ or 1 .

Let $\mathfrak{H}$ be a Hall subgroup of $\mathfrak{G}$ which is a product of $\langle\zeta\rangle$ and a 2Sylow subgroup of $\mathfrak{G}$. As $a \geq 2, \mathfrak{H}$ is not a subgroup of $\langle\zeta, \sigma\rangle$. That is to say, $\langle\zeta\rangle$ is not normal in $\mathfrak{H}$. Let $\mathfrak{A}$ be a maximal abelian normal subgroup of the solvable group $\mathfrak{H}$.

- The group $\mathfrak{A}$ is a 2-group :

For otherwise the unique 7-Sylow subgroup of $\mathfrak{A}$ would be normal in $\mathfrak{H}$.

- The group $\mathfrak{A}$ is the unique 2 -Sylow subgroup of $\mathfrak{H}$ :

As $\mathfrak{A}$ is a 2-group, the subspace $E^{\mathfrak{A}}$ of $E$ consisting of all vectors fixed by $\mathfrak{A}$ is a non-zero $\mathfrak{H}$-module. So $E^{\mathfrak{A}}$ is either $V$ or $V^{\vee}$. Replacing $\mathfrak{G}$ by $\sigma \mathfrak{G} \sigma^{-1}$ if necessary, we suppose $E^{\mathfrak{A}}=V$. Thus $\mathfrak{A}$ is a subgroup of S. Notice that $\sigma^{3}$ does not normalize $V$. So $\mathfrak{H} \cap\langle\zeta, \sigma\rangle=\langle\zeta\rangle$ and so $\mathfrak{H}$ has $2^{a}=|\mathfrak{H} /\langle\zeta\rangle| 7$-Sylow subgroups. Then $\mathfrak{H}$ has a unique 2-Sylow subgroup, say $\mathfrak{a}$, because $2^{a} .7-2^{a}(7-1)=2^{a}$. Then $E^{\mathfrak{a}}$ is a non-zero 
sub- $\mathfrak{H}$-module of $E^{\mathfrak{A}}=V$. So $E^{\mathfrak{a}}=V$. So $\mathfrak{a} \leq \mathfrak{S}$. Thus $\mathfrak{a}$ is abelian. One concludes that $\mathfrak{A}=\mathfrak{a}$.

In particular, $\mathfrak{H} \leq\langle\zeta\rangle \mathfrak{S}$ and $\mathfrak{G}=\mathfrak{L} \mathfrak{H} \leq\left\langle\zeta, \sigma^{2}\right\rangle \mathfrak{S}$.

To finish, it suffices to show that both $\langle\zeta\rangle \mathfrak{S}^{1}$ and $\langle\zeta\rangle \mathfrak{S}^{2}$ act transitively on $X$. Both have 56 elements. And it is immediate to verify that each intersects $\mathrm{O}(q)$ in two elements.

Proposition 12.2. Let $(S, \eta, s), \operatorname{char}(s)=\ell$, be as in $\S 4$. Then $\left(E_{7}, \alpha_{7}\right)$ is elliptic over $\eta$ if and only if $\ell=2$.

Proof. By (12.1) all solvable subgroup of $\{1,-1\} \times \operatorname{Sp}(E)$ that act transitively on

$$
\{1,-1\} \times(\operatorname{Sp}(E) / \mathrm{O}(q))=\{1,-1\} \times X
$$

contain elementary 2 -groups of 2 -rank $\geq 3$. So $\left(E_{7}, \alpha_{7}\right)$ is not elliptic if $\ell>2(3.1), 7)$.

Suppose $\ell=2$. Then $\mathfrak{G}:=\{1,-1\} \times\langle\zeta\rangle \mathfrak{S}$ is a quotient of $\pi_{1}(\eta, \bar{\eta})$ by (4.1) and because $\langle\zeta\rangle \mathfrak{S}$ has no index 2 subgroups. Moreover, $\mathfrak{G}$ acts transitively on $\{1,-1\} \times X(12.1)$. So $\left(E_{7}, \alpha_{7}\right)$ is elliptic when $\ell=2$ $(3.1), 7)$.

\section{REFERENCES}

1. J.D. Achter. Split reductions of simple abelian varieties. Math. Res. Lett. 16, no. 2 : 199-213, 2009.

2. F. Bogomolov. Sur l'algébricité des représentations $\ell$-adiques. Comptes Rendus Acad. Sci. Paris, 290, 701-703, 1980.

3. N. Bourbaki. Groupes et algèbres de Lie. Hermann, 1975.

4. P. Deligne. Hodge cycles on abelian varieties. Hodge Cycles, Motives, and Shimura Varieties, LNM 900.

5. G. Faltings. Endlichkeitssätze für abelsche Varietäten über Zahlkörpern. Invent. Math, 73, 349-366, 1983.

6. R. Howe. Perspectives on Invariant Theory. Israel Mathematical Conference Proceedings, vol $8: 1-182,1995$.

7. D. Livingstone and A. Wagner. Transitivity of finite permutation groups on unordered sets. Math. Z, 90 : 393-403, 1965.

8. V. Kumar Murty and Vijay. M. Patankar. Splitting of abelian varieties. Int. Math. Res. Not, 2008.

9. J.P. Serre. Corps locaux. Publ. Math. Nancago, Hermann, 1962.

10. J.P. Serre. Abelian l-adic Representations and Elliptic Curves. Benjamin, 1968.

11. J.P. Serre. Cohomologie Galoisienne. LNM 5, cinquième edition, 1997.

12. J. Tate. Endomorphisms of abelian varieties over finite fields. Invent. Math, 2, 134-144, 1966. 
13. J. Tate. Classes d'isogénie de variétés abéliennes sur un corps fini (d'après T.Honda). Séminaire Bourbaki, 1968/69, Exp. 352, LNM 179 : 95-110, 1971.

14. J. Tits. Classification of Algebraic Semisimple Groups. Algebraic Groups and Discontinuous Subgroups, Proc. Sympos. Pure Math : 33-62, 1966.

Department of Mathematics, University of Toronto

E-mail address: murty@math.toronto.edu, zongying@math.toronto.edu 